\title{
International hearing protector standardization
}

\author{
Poulsen, Torben
}

Published in:

Acoustical Society of America. Journal

Publication date:

2002

Document Version

Publisher's PDF, also known as Version of record

Link back to DTU Orbit

Citation (APA):

Poulsen, T. (2002). International hearing protector standardization. Acoustical Society of America. Journal, 112(5), 2293-2293.

\section{General rights}

Copyright and moral rights for the publications made accessible in the public portal are retained by the authors and/or other copyright owners and it is a condition of accessing publications that users recognise and abide by the legal requirements associated with these rights.

- Users may download and print one copy of any publication from the public portal for the purpose of private study or research.

- You may not further distribute the material or use it for any profit-making activity or commercial gain

- You may freely distribute the URL identifying the publication in the public portal

If you believe that this document breaches copyright please contact us providing details, and we will remove access to the work immediately and investigate your claim. 


\title{
Session 3aAA
}

\section{Architectural Acoustics, Musical Acoustics and Physical Acoustics: Ancient Acoustics I Megaliths and Pyramids}

\author{
David Lubman, Cochair \\ David Lubman \& Associates, 14301 Middletown Lane, Westminster, California 92683 \\ Fernando J. Elizondo, Cochair \\ Acoustics Laboratory, FIME, Univ. A de Nuevo Leon, P.O. Box 28 "F," Cd. Universitaria, \\ San Nicolas 66450, N.L. Mexico \\ Chair's Introduction-8:30 \\ Invited Papers
}

8:35

3aAA1. Psychoacoustic influences of the echoing environments of prehistoric art. Steven J. Waller (Rock Art Acoust., 5381 Wellesley St., La Mesa, CA 91942, wallersj@yahoo.com)

Cave paintings and ancient petroglyphs around the world are typically found in echo rich locations such as caves, canyons, and rocky cliff faces. Analysis of field data shows that echo decibel levels at a large number of prehistoric art sites are higher than those at nondecorated locations. The selection of these echoing environments by the artists appears not to be a mere coincidence. This paper considers the perception of an echoed sound as a psychoacoustic event that would have been inexplicable to ancient humans. A variety of ancient legends from cultures on several continents attribute the phenomenon of echoes to supernatural beings. These legends, together with the quantitative data, strongly implicate echoing as relevant to the artists of the past. The notion that the echoes were caused by spirits within the rock would explain not only the unusual locations of prehistoric art, but also the perplexing subject matter. For example, the common theme of hoofed animal imagery could have been inspired by echoes of percussion noises perceived as hoof beats. Further systematic acoustical studies of prehistoric art sites is warranted. Conservation of the natural acoustic properties of rock art environments—a previously unrecognized need—is urged.

8:55

3aAA2. MegaSound: Sound in Irish megalithic buildings. Victor Reijs (Geniet, 15 Shenick Grove, Skerries, Dublin, Ireland, geniet@iol.ie)

Stimulated by the studies done by Paul Deveraux and Robert Jahn, research has been conducted on the sound properties of two megalithic chambers is Ireland: Dowth South and Fourknocks I. As reference measurements two normal rooms (bed- and bathroom) have been studied. The following aspects will be covered in the presentation: some theoretical background on acoustical modes (within a passage, a chamber, and a combination of them: Helmholtz resonator); tips for doing sound experiments inside megalithic chambers (like: equipment, measurement software, power provisioning and calibrating); frequency response measurements (between 20 and $200 \mathrm{~Hz}$ ) for the surveyed chambers/rooms; comparison of the results with other researchers' results; background on the pitch of the human (male, female, and child) voices in neolithic times and recommendations for future research. The presentation also provides insight in the aeralization (simulation) of sound in a megalithic chamber, covering: software that can do these simulations; issues in finding the basic information, e.g., acoustic absorption coefficients and provide examples of the results. I would like to thank all the people who have provided constructive feedback on my work (http://www.iol.ie/ geniet/eng/megasound.htm).

9:15

3aAA3. The accidental (acoustical) tourist. Wayne Van Kirk (7368 Brace St., Houston, TX 77061)

The acoustical phenomenon observed at an ancient temple in the Great Ball Court at Chichen Itza was described as "little short of amazing - an ancient whispering gallery" by Silvanus G. Morley, leader of the Carnegie Institute's archaeological team that excavated and restored these structures in the 1920s. Since then, many others have experienced the extraordinary acoustics at Chichen Itza and other Maya sites. Despite these reports, archaeologists and acousticians have until recently shown little interest in understanding these phenomena. After experiencing Chichen Itza's remarkable acoustics as a tourist in 1994, the author commenced collecting and disseminating information about acoustical phenomena there and at other Mayan sites, hoping to stimulate interest among archaeologists and acousticians. Were these designs accidental or intentional? If intentional, how was the knowledge obtained? How were acoustical features used? This paper highlights the author's collection of anecdotal reports of mysterious Mayan acoustics (http://www.ianlawton.com/pal.htm), recommended reading for scientists and engineers who wish to pursue this fascinating study. Also recounted are some of the reactions of archaeologists-ranging from curious, helpful, and insightful to humorous and appalling - to outsiders' efforts to bring serious scientific attenation to the new field of acoustical archaeology. 
3aAA4. Tonal response on the stairway of the main pyramid at La Ciudadela, Teotihuacan archaeological site. Sergio Beristain, Cecilia Coss, Gabriela Aquino, Jose Negrete (P.O. Box 75805, Mexico, D. F. C.P. 97300, Mexico, sberista@ hotmail.com), and Pablo Lizana (ESIME, IPN, Mexico)

This paper presents new research on the very interesting audible effects produced by the stairways of many archaeological sites in Mexico. This investigation was made at the main stairway of the pyramid at La Ciudadela, Teotihuacan archaeological site. The effect previously studied was a chirped echo reflected from the stairway at normal incidence, which resembles the singing of the Quetzal. Now it is presented with the impulsive sound source and the listeners located at different angles, where apart from the characteristic chirped sound, several musical notes could be obtained and identified, covering a range of at least one half an octave. This evaluation was made at the site, where the effect is clearly audible, and it is supported with simple mathematics.

\section{9:55-10:05 Break}

\section{0:05}

3aAA5. Acoustical features of two Mayan monuments at Chichen Itza: Accident or design? David Lubman (David Lubman \& Assoc., 14301 Middletown Ln., Westminster, CA 92683-4514, dlubman@ix.netcom.com)

Chichen Itza dominated the early postclassic Maya world, ca. 900-1200 C.E. Two of its colossal monuments, the Great Ball Court and the temple of Kukulkan, reflect the sophisticated, hybrid culture of a Mexicanized Maya civilization. The architecture seems intended for ceremony and ritual drama. Deducing ritual practices will advance the understanding of a lost civilization, but what took place there is largely unknown. Perhaps acoustical science can add value. Unexpected and unusual acoustical features can be interpreted as intriguing clues or irrelevant accidents. Acoustical advocates believe that, when combined with an understanding of the Maya worldview, acoustical features can provide unique insights into how the Maya designed and used theater spaces. At Chichen Itza's monuments, sound reinforcement features improve rulers and priests ability to address large crowds, and Ball Court whispering galleries permit speech communication over unexpectedly large distances. Handclaps at Kukulkan stimulate chirps that mimic a revered bird ("Kukul"), thus reinforcing cultic beliefs. A ball striking playing field wall stimulates flutter echoes at the Great Ball Court; their strength and duration arguably had dramatic, mythic, and practical significance. Interpretations of the possible mythic, magic, and political significance of sound phenomena at these Maya monuments strongly suggests intentional design.

\section{Contributed Papers}

10:25

3aAA6. Quetzal or not Quetzal, that is the question ... . On the stairs of the Castillo monument in Chichen Itza. Fernando J. Elizondo-Garza (Acoust. Lab., FIME, Univ. A. de Nuevo Leon, P.O. Box 28 F, Cd. Universitaria, San Nicolas 66450, N.L. Mexico, fjelizon@ccr.dsi.uanl.mx)

Some speculation will be presented about the hypothesis that states that the reflected sounds in the stairs of the Castillo building in Chichen Itza, Mexico, imitates the song of the quetzal bird. Some aspects of construction, both technical and social, are discussed as well as issues related with myth and fantasies.

\section{0:40}

3aAA7. Theoretical interpretation of a case study: Acoustic resonance in an archaeological site. Jorge Carrera (Eng. Faculty, UNAM, Mexico, jorgec00@yahoo.com) and Sergio Beristain (ESIME, IPN, Mexico)

It is well-known that the stairways of some Mexican archaeological sites, like Chichen-Itza or Teotihuacan, present an interesting sound reflection and resonance phenomenon which causes a special audible effect. In this paper, mathematical modeling of this situation is presented, and the practical phenomenon is discussed from a theoretical standpoint. More than an end in itself, the idea is, once this validated model is obtained, to use the results for the analysis of a more extensive architectural environment in order to establish whether this kind of phenomenon would have been purposely introduced in the design of the site. This will be presented in future publications.

\section{0:55}

3aAA8. Navajo oral history of a pre-Columbian amphitheater in Chaco Canyon, New Mexico. Taft Blackhorse, Jr. (Navajo Nation Historic Preservation Dept., P.O. Box 4950, Window Rock, AZ 86515) and Jay S. Williams (Univ. of New Mexico, Albuquerque, NM 87108)

A large performance space attached to a natural amphitheater in the cliff face has recently been identified at the center of the pre-Columbian Chaco Complex in Chaco Canyon, New Mexico. This location is known in the ceremonial history of the Navajo people (Din) as Tsbiinaholtsa Yalti
(Concavity in Bedrock that Speaks). Tsbiinaholtsa Yalti is a portal to the dimension of the deities and it is opened by way of tonally induced acousma. The physical manifestation of the amphitheater and its acoustical properties invoke the concepts of Tal (Chants) and Taal (Ceremonial pathway). Navajo ceremonies are called Haataal and religious practitioners Hataalii (Chanters). The origin of the tones which give power to contemporary Navajo chants may be traced to the Tsbiinaholtsa Yalti. These tones are produced vocally and are accompanied by the shell trumpet, eagle bone whistle, and reed flute (jadzoosh).

\section{1:10}

3aAA9. Computer analysis of sound recordings from two Anasazi sites in northwestern New Mexico. Richard Loose (MEVATEC Corp., P.O. Box 1419, Las Cruces, NM 88004)

Sound recordings were made at a natural outdoor amphitheater in Chaco Canyon and in a reconstructed great kiva at Aztec Ruins. Recordings included computer-generated tones and swept sine waves, classical concert flute, Native American flute, conch shell trumpet, and prerecorded music. Recording equipment included analog tape deck, digital minidisk recorder, and direct digital recording to a laptop computer disk. Microphones and geophones were used as transducers. The natural amphitheater lies between the ruins of Pueblo Bonito and Chetro Ketl. It is a semicircular arc in a sandstone cliff measuring $500 \mathrm{ft}$. wide and $75 \mathrm{ft}$. high. The radius of the arc was verified with aerial photography, and an acoustic ray trace was generated using CAD software. The arc is in an overhanging cliff face and brings distant sounds to a line focus. Along this line, there are unusual acoustic effects at conjugate foci. Time history analysis of recordings from both sites showed that a $60-\mathrm{dB}$ reverb decay lasted from 1.8 to $2.0 \mathrm{~s}$, nearly ideal for public performances of music. Echoes from the amphitheater were perceived to be upshifted in pitch, but this was not seen in FFT analysis. Geophones placed on the floor of the great kiva showed a resonance at $95 \mathrm{~Hz}$. 


\title{
Session $3 \mathrm{aAB}$
}

\section{Animal Bioacoustics and Psychological and Physiological Acoustics: Electrophysiological Investigations of Animals I}

\author{
Eduardo Mercado III, Chair \\ Center for Molecular and Behavioral Neuroscience, Rutgers University, 197 University Avenue, Newark, New Jersey 07102
}

Invited Papers

8:00

3aAB1. Noise changes receptive fields of auditory neurons in A1. David T. Blake and Michael M. Merzenich (513 Parnassus Ave. S-877, San Fransisco, CA 94143-0732)

Primates engage in auditory behaviors under a broad range of signal to noise conditions. In this study, optimal linear receptive fields were measured in alert primate A1 in response to stimuli that vary in spectrotemporal density. As the random tone pip density increased, selective A1 excitatory receptive fields systematically changed. Receptive field sensitivity, expressed as the expected change in firing rate after a tone pip onset, decreased by an order of magnitude. Spectral selectivity more than doubled. Inhibitory subfields, which were rarely recorded at low sound densities, emerged at higher sound densities. The ratio of excitatory to inhibitory population strength changed from 14.4:1 to 1.4:1. At low sound densities, the sound associated with the evocation of an action potential from an A1 neuron was broad in spectrum and time. At high sound densities, a spike-evoking sound was more likely to be a spectral or temporal edge, and was narrower in both time and frequency range. Prediction experiments were performed to validate the assumption that linear receptive fields were representative of neural responses at high noise densities. The auditory context alters A1 responses across multiple parameter spaces; this presents a challenge for reconstructing neural codes.

8:20

3aAB2. Psychophysical and physiological measures of spectro-temporal processing in humans. David A. Eddins and Ann C. Eddins (Ctr. for Hearing and Deafness, Dept. of Communicative Disord. and Sci., Univ. at Buffalo, Buffalo, NY 14214)

The normal perception of natural acoustic stimuli requires the brain to extract, encode, and interpret multidimensional patterns derived from the acoustic input as relayed by the auditory periphery. Of particular importance in audition are patterns of intensity variations across time and frequency. To better understand the relation between perceptual abilities and underlying physiological mechanisms, one may study the relation between measures of psychophysical performance and physiological coding in humans on a range of auditory tasks. Furthermore, similar physiological measures are easily obtained in a variety of animal species, providing a critical link between human perception and relevant animal models. The first series of experiments to be reviewed involves behavioral and physiological measures of auditory temporal processing including the perception of sinusoidal amplitude modulation and nonsimultaneous masking. The second series involves the perception of spectral envelope features using behavorial and physiological estimates of the spectral modulation transfer function. The ability to combine or compare temporal patterns across different spectral regions will be examined via the comodulation masking release (CMR) paradigm. Finally, the processing of local and global spectral features will be examined and hemispheric dominance for each will be discussed.

\section{8:40}

3aAB3. Physiological measures of auditory temporal integration in chinchillas and humans. Ann Clock Eddins and David A. Eddins (Dept. of Communicative Disord. \& Sci., Ctr. for Hearing and Deafness, Univ. at Buffalo, 215 Parker Hall, Buffalo, NY 14214)

Temporal integration is a phenomenon in which signal detection improves with increasing signal duration. The magnitude of threshold improvement has been shown to vary as a function of signal frequency and is often diminished in the presence of hearing loss. In studies of chinchilla auditory nerve fibers and cochlear nucleus $(\mathrm{CN})$ neurons, we have shown that single neuron thresholds improve with increasing duration in a manner similar to data from psychophysical studies. Moreover, it appears that a critical feature of duration coding in the $\mathrm{CN}$ is the pattern of spike activity rather than the total number of spikes over time. Thresholds measured in evoked-potential studies with chinchillas at the level of the inferior colliculus and with humans at the level of the cortex also reflect physiological evidence of temporal integration, with comparable frequency effects and degradation with hearing loss like that observed psychophysically. Although frequency effects that show greater threshold improvement for low versus high frequencies have been reported in the literature for decades, the underlying mechanism remains unclear and is under further investigation psychophysically and physiologically in our laboratories. The results of these investigations will be discussed along with potential underlying mechanisms. 
3aAB4. The effects of selective inner hair cell loss on auditory evoked potentials recorded from multiple levels of the auditory system of the unanesthetized chinchilla. Robert Burkard (Ctr. for Hearing \& Deafness, Univ. at Buffalo, 215 Parker Hall, Buffalo, NY14214, RFB@acsu.buffalo.edu)

Most animal models of sensorineural hearing loss show either selective outer hair cell (OHC) loss, or combined OHC and inner hair cell (IHC) loss. Several years ago, Robert Harrison and colleagues discovered that carboplatin could produce a selective IHC loss in the chinchilla. Carboplatin is a second-generation, platinum-based anti-neoplastic agent. In chinchillas, the effect of carboplatin administered systemically is dose dependent, and for the appropriate dosage, produces selective, patchy IHC loss that extends the length of the cochlea. As the vast majority of auditory-nerve afferents innervate the IHCs, this patchy IHC loss due to carboplatin leads to a partial deafferentation of the cochlea. This invited presentation will review a series of studies investigating near-field auditory evoked potentials in the chinchilla that have been performed at the University at Buffalo by the author and his colleagues. Chronic electrode implantation combined with passive animal restraint allow us to record from unanesthetized animals, thus avoiding the confounding effects of anesthesia. Responses from multiple levels of the nervous system (e.g., round window, auditory nerve, inferior colliculus, auditory cortex) will be reported, comparing responses before and after inducing partial IHC loss. [Work supported by NIH NICDC DC03600.]

9:20

3aAB5. Neural response characteristics in auditory cortex of the awake ferret. Didier A. Depireux, Bing-Zhong Chen, Peter Marvit, and Yaan Li (Univ. of Maryland, Baltimore, 685 S. Baltimore St., HSF 222, Baltimore, MD 21201)

We are developing an awake preparation for chronic physiological recording in the ferret. In this talk, we describe neural responses in auditory cortex in the restrained preparation to a variety of acoustic stimuli including tones, noise, auditory gratings, and their combinations. We characterize population responses, show how the cells properties change as a function of the stimulus (e.g., how the receptive field of a cell changes, depending on whether the carrier under the gratings spectral envelope is a harmonic complex or noise) and correlated the activity of single units from neighboring cells in a cortical column in response to these stimuli. [Work supported in part by NIH Grant No. R01 DC05019-01A1 and a training grant to the Program in Neuroscience at UMAB.]

\section{9:40}

3aAB6. Models of birdsong learning. Daniel Margoliash (Dept. of Organismal Biol. \& Anatomy, Univ. of Chicago, Chicago, IL 60637, dan@ bigbird.uchicago.edu)

The study of birdsong learning and the passerine song system represents an excellent opportunity to understand how complex acoustic perceptions are represented in the brain, and how these interact with motor systems to produce learned behavior. Recent advances suggesting specific functional roles for different nuclei within the song system help to constrain proposed models of the sensorimotor phase of learning. In particular, a descending motor pathway organized in a temporal hierarchy is involved in motor programming whereas an anterior forebrain basal ganglialike pathway may provide auditory and motor feedback-mediated error signals to adjust the motor pathway. A separate pathway outside of the song system may be devoted to perception of conspecific songs. Central to the problem of vocal learning is the time delay between motor command and auditory feedback. Both real time online models and offline models have been proposed as solutions to the temporal credit assignment problem. The models differ in the types of synaptic mechanisms envisioned that involve forward predictions, and the relative importance of state dependency of song system activity and song learning. Recent data will be given that describe the insight into neural solutions to these problems.

10:00-10:10 Break

10:10

3aAB7. Audiomotor integration for active sensing in the echolocating bat, Eptesicus fuscus. Cynthia Moss and Shiva Sinha (Dept. of Psych., Inst. for Systems Res., Univ. of Maryland, College Park, MD 20742)

Echolocation depends upon the dynamic interplay between auditory information processing and adaptive motor control. We are conducting experiments aimed at understanding the neural mechanisms supporting audiomotor integration for echolocation in the big brown bat, Eptesicus fuscus. In this work we focus on the superior colliculus (SC), a midbrain structure implicated in species-specific orienting behaviors. We have characterized the spatial response profiles of auditory neurons in the bat SC, and have discovered a population of cells that show echo-delay tuning, a response property believed to encode target distance in the bats sonar receiver. These data reveal a 3-D spatial coordinate system that may be used to guide appropriate orienting responses. Our studies also demonstrate that the bat SC plays a functional role in the execution of motor commands used for acoustic orientation by sonar. In particular, SC microstimulation elicits head and pinna movements, along with the production of sonar vocalizations. Multi-unit recordings from the SC of tethered, vocalizing bats reveal bursts of neural activity preceding the production of each sonar cry. Collectively, these results suggest that the bat SC plays a functional role in both auditory information processing and orienting behaviors that operate together in echolocation. [Work supported by the NSF, the NIMH, and the Whitehall Foundation.]

$10: 30$

3aAB8. Neuroethology of audition and bat evasion in praying mantises. David D. Yager (Dept. of Psych., Univ. of Maryland, College Park, MD 20742, dy5@umail.umd.edu)

Many praying mantises have a unique auditory system comprising a single ear located in the ventral midline of the thorax. The hearing is nondirectional, and best frequencies range from $25 \mathrm{kHz}$ to over $100 \mathrm{kHz}$ depending on the species. Bat-like ultrasound triggers complex evasive maneuvers in flying mantises that requires CNS processing in the head. A mirror-image pair of interneurons 
(501) rapidly $(<20 \mathrm{~ms})$ carries auditory information from the auditory nerve to the brain and these neurons are the best candidates as the primary input to the evasive response. The 501 recordings from an electrode chronically implanted in a mantis while it is being attacked by a flying, echolocating bat show that 501 responds strongly to bat cries and faithfully reports the temporal pattern of the echolocation cries during the early stages of the attack. However, during the last 200-250 ms before capture (beginning in mid-Buzz I), 501 falls silent. Although consistent with several physiological characteristics of 501, the result is surprising behaviorally. However, based on behavioral latencies, the latest that a successful response could be triggered is 200-250 ms before capture, and silencing 501 after that may prevent potentially dangerous habituation of the interneuron.

\section{0:50}

3aAB9. Time and frequency information processing in the midbrain: Evidence found in the gerbil inferior colliculus. Hiroshi Riquimaroux and Katuhiro Maki (Dept. of Knowledge Eng. and Computer Sci., Doshisha Univ., Japan)

We have investigated the firing pattern of neurons in the central nucleus of the inferior colliculus (ICc) in time-frequency coordinate. The ICc is the major nucleus in the auditory midbrain. Single-unit recordings were made from ICc contralateral to the monaurally stimulated ear in anesthetized gerbils. We could classify firing patterns into four groups, except the simple ON neurons. Three out of four groups of neurons (53.8\%) demonstrated that firing distribution changed depending on time and frequency, which was not shown previously. One group (37.6\%) exhibited a frequency response range that changed little with time. The remainder (8.6\%) belonged to none of the above. The time-frequency sensitive neuron in ICc may be a good candidate to code communication sounds, which comprise complex temporal change in frequency. [Research supported by Special Coordination Funds from the Science and Technology Agency and a grant to RCAST at Doshisha University from the Ministry of Education and Science of Japan.]

11:10

3aAB10. FM signals produce a robust paradoxical latency shift that is important for coding of target range in the bat inferior colliculus. Albert S. Feng and Alexander Galazyuk (Dept. of Molec. \& Integ. Physiol. and Beckman Inst., Univ. of Illinois, 405 N. Mathews, Urbana, IL 61801)

Echolocating bats utilize the time delay between an outgoing ultrasonic pulse and its echo to determine target range. Many neurons at the inferior colliculus (IC) and above are tuned to time delays between these sound pulses of unequal amplitudes. Sullivan previously proposed that paradoxical latency shift (PLS), characterized by a quantal increase in firing latency to loud sounds, is important for this attribute because PLS permits coincidence detection that is important for the creation of delay-tuned responses. In the IC of little brown bats, Galazyuk and Feng recently reported that, in response to tone pulses, the proportion of neurons showing PLS was low $(<20 \%)$. This study was undertaken to determine whether PLS is a function of the acoustic stimulus. For this, the temporal discharge patterns of single IC neurons were investigated over a broad range of sound levels, using tone pulses at CF as well as FM sound pulses that mimicked bats' ultrasonic cry as stimuli. For many IC neurons, tone pulses did not elicit PLS but FM sound pulses produced robust PLS. This result showed that PLS is stimulus dependent and that the bat's auditory system is optimal for processing FM sounds employed during echolocation. [Work supported by NIH R01DC04998.]

\section{1:30}

3aAB11. Phase sensitivity of auditory brain-stem responses in echolocating big brown bats. Michael J. Ferragamo (Dept. of Biol., Gustavus Adolphus College, St. Peter, MN), Mark I. Sanderson, and James A. Simmons (Brown Univ., Providence, RI 02912)

Multiple behavioral experiments show that echolocating big brown bats perceive $180^{\circ}$ phase shifts of ultrasonic $(20-100 \mathrm{kHz}) \mathrm{FM}$ echoes as delay changes of $\pm 15 \mu \mathrm{m}$. These bats represent FM sweeps as coherent auditory spectrograms in which low-pass smoothing of half-wave-rectified hair-cell excitation is the critical limiting parameter. Computational modeling of auditory spectrograms combined with Monte Carlo simulation of echo delay psychophysics reveals that coherence is preserved when the auditory lowpass smoothing cutoff is as low as $7-10 \mathrm{kHz}$, which is not nearly as high as the 20- to $50-\mathrm{kHz}$ ultrasonic frequencies that seem necessary intuitively. Local-field-potential recordings from the bat's auditory brain-stem are sensitive to the starting phase of tonebursts at frequencies up to $14 \mathrm{kHz}$, manifested as a change in LFP wave-form shape with phase. For most sites this phase sensitivity exhibits a strong dependence on stimulus amplitude. Typically, tone-bursts of 65-75 dB SPL evoke significant changes in LFP responses across different phase conditions. These results appear to confirm that low-pass smoothing in the bat's auditory transduction process occurs at higher frequencies than for most mammals, and at frequencies that have the ability to confer coherence on the resulting auditory representation of biosonar echoes.

\section{1:50}

3aAB12. Genesis of a space map in the owl's brain. Jose L. Pena (Dept. of Biol., 216-76, Caltech, Pasadena, CA 91125, jose@etho.caltech.edu)

In order to localize a sound source, the brain uses monaural and binaural cues that convey spatial information. In the owl, space-specific neurons of the inferior colliculus respond only to sounds coming from a particular direction and represent the results of this computation. The parallel pathways that process interaural time difference (ITD) and interaural level difference (ILD) merge in the external nucleus of the inferior colliculus where the space-specific neurons are selective to combinations of ITD and ILD. How is the combination selectivity to ITD-ILD pairs achieved? A multiplication of postsynaptic potentials tuned to ITD and ILD can account for the response of these neurons to ITD-ILD pairs. There are very few examples of multiplication by neurons or neural circuits; however, some computational models assume the existence of this basic arithmetic in sound localization. The owl's auditory system uses such operation in creating a two-dimensional map of auditory space. 


\title{
Session 3aBB
}

\section{Biomedical Ultrasound/Bioresponse to Vibration: Lithotripsy I}

\author{
Robin O. Cleveland, Cochair \\ Aerospace and Mechanical Engineering, Boston University, 110 Cummington Street, Boston, Massachusetts 02215 \\ Achim M. Loske, Cochair \\ Centro de Física Aplicada y Technología Avanzada, UNAM, A.P. 1-1010, Querétaro, Qro. Mexico
}

Chair's Introduction-8:25

Invited Papers

8:30

3aBB1. A new fragmentation mechanism in extracorporeal shock wave lithotripsy and a first clinical study in China. Wolfgang Eisenmenger (Physikalisches Institut, Universitt Stuttgart, Pfaffenwaldring 57, D-70550 Stuttgart, Germany)

With ESWL-focus diameters of the order of the stone dimension or larger, fragmentation in planes perpendicular and parallel to the wave plane is observed. This is explained by circumferential pressure or "squeezing" of the stone by the wave propagating at the outside of the stone in the liquid or tissue. Since the pressure zone propagates with the sound velocity in the liquid which is below the propagation velocity in the stone, it causes an evanescent pressure zone in the stone resulting in tensile stress in planes perpendicular and parallel to the wave plane. A quantitative model predicting the ratio of pulses needed to fragment the stone to 2-mm particle size in relation to the number of pressure pulses needed for the first fragmentation is well in accord with experiments, supporting the "squeezing mechanism with binary fragmentation." On the basis of these results it now appears possible to optimize the pressure pulse parameters measured using the fiber-optic probe hydrophone (FOPH). With correspondingly optimized shock wave generator systems, a clinical study of the concept "wide focus and low pressure" ESWL was performed in scientific cooperation between the Physical Institute of the University of Stuttgart and the Xixin Medical Instruments Co., Ltd. in Suzhou, China. [W. Eisenmenger, "The mechanism of stone fragmentation in ESWL," Ultrasound Med. Biol. 27, 683-693 (2001); Eisenmenger, Du, Tang et al., "The first clinical results of wide focus and low pressure ESWL," ibid. 8, 769-774 (2002) (in press).]

9:00

3aBB2. The relative contribution of stress waves and cavitation to the overall success of stone comminution in shock wave lithotripsy. Pei Zhong, Songlin Zhu, and Yufeng Zhou (Dept. of Mech. Eng. and Mat. Sci., Duke Univ., Box 90300, Durham, NC 27708)

The disintegration of kidney stones in shock wave lithotripsy (SWL) is caused primarily by the stress waves propagating inside the stone and by the collapse of cavitation bubbles in the surrounding fluid near the stone surface. Understanding the relative contribution of these different mechanical forces to the overall success of stone comminution is critical for improving the treatment efficiency of SWL. In this talk, we will provide an overview of the experimental studies under way at Duke University to delineate the contribution of stress waves and cavitation to the overall success of stone comminution in SWL. Limitations of individual contributing factors and their synergistic interaction will also be discussed. Finally, strategies to improve stone comminution efficiency while reducing tissue injury in SWL will be presented. [Work supported by NIH DK52985 and DK58266.]

9:20

3aBB3. Compact self-focusing piezoelectric shock wave generator using electrically prestressed transducer. Dominique Cathignol, Alain Birer, and Mohammad Ghohestani (INSERM Unite 556, 151 Cours Albert Thomas, 69424 Lyon Cedex 03, France)

Compact piezoelectric shock heads need to increase the pressure at the surface of the shell. However, the pressure is limited by the possible mechanical breakdown. So, we have proposed to electrically pre-stress the piezoelectric material by applying an electric field in the opposite direction of the polarization. Using this method, it was possible to reach, on a plane piston, a $5 \mathrm{MPa}$ pressure value. According to this idea, a very compact shock wave generator made of a 1-3 piezocomposite material having a diameter and a focal length of $120 \mathrm{~mm}$ was developed. The maximum pressure and the width of the compressive wave at the focus were, respectively, 60 $\mathrm{MPa}$ and 1.5 microseconds. The focal zone is an ellipsoid $6 \mathrm{~mm}$ in the propagating axis and $3 \mathrm{~mm}$ in the perpendicular direction. The efficacy of this generator was measured as the number of shocks necessary to disintegrate plaster balls $15 \mathrm{~mm}$ in diameter. At full power, the number of shocks was only 150 which is rather the same number as the one obtained using electrohydraulic machine generally considered as the gold standard. These results show that piezoelectric material may be advantageously used for the manufacturing of shock wave generators. 
3aBB4. Dual-pulse lithotripter accelerates stone comminution and reduces cell injury in vitro. Dahlia L. Sokolov, Michael R. Bailey, and Lawrence A. Crum (Ctr. for Industrial and Medical Ultrasound, Appl. Phys. Lab., 1013 NE 40th St., Seattle, WA 98105)

Peak acoustic pressures and cavitation generated in shock wave lithotripsy (SWL) appear to contribute to both desired stone comminution and undesired injury to surrounding renal tissue. Our dual pulse system, comprised of two opposing, confocal lithotripters and generating simultaneous, converging shock pulses, localizes and intensifies the peak pressures and cavitation. Comparison of cavitation damage to aluminum foil shows an $8-\mathrm{cm}$ stripe of pits produced by a single-pulse lithotripter and a 1-cm stripe of deep pits produced by the dual-pulse lithotripter. 100 dual pulses generated at $15 \mathrm{kV}$ comminuted gypsum stones placed at the geometric focus $F 2$ into 8 times as many fragments and significantly reduced hemolysis in dilute blood 2 and $4 \mathrm{~cm}$ off $F 2$ when compared to 200 single pulses generated at $18 \mathrm{kV}$. Thus the dual-pulse lithotripter enhanced comminution and reduced injury while cutting treatment time in half. Additionally, when cavitation was suppressed by placing the stones in glycerol, the improvement in comminution was reduced to only a twofold increase. This result indicates that the localized and intensified cavitation is the dominant mechanism in the accelerated comminution produced by the dual-pulse lithotripter. [Work supported by NIH Grants Nos. P01DK43881 and R01-DK55674.]

\section{0:00-10:20 Break}

\section{0:20}

3aBB5. Lithotripter shock wave interaction with bacteria. Achim M. Loske (Centro de Física Aplicada y Tecnología Avanzada, UNAM A.P. 1-1010, Querétaro, Qro., México, loske@ fisica.unam.mx

The worldwide success of extracorporeal lithotripsy and shock wave treatment of some orthopedic diseases is not doubted. In the future, shock waves could also be used as a method for in vivo drug delivery and gene transfer into target cells; however, basic research is still necessary to understand shock wave interaction with biological tissue, bacteria, and cells. The destructive effects of ultrasonic waves on micro-organisms and the reduction in renal infections after extracorporeal shock wave lithotripsy led to the idea of studying the effects of lithotripter shock waves on bacteria. Independent of possible applications to medicine, this could lead to a new, nonthermal food preservation method. The bactericidal effect of underwater shock waves on Escherichia coli ATCC 10536 and O157:H7, Listeria monocytogenes L8, and Salmonella enterica servovar typhimurium suspensions in isotonic saline solution were studied. Shock waves of about $55 \mathrm{MPa}$ were generated using an electrohydraulic generator. Results indicate that pressure variations, shock wave-created cavitation, and the radiation resulting from the high-voltage spark significantly reduce the viability of these micro-organisms. An analysis of variance revealed that the bactericidal action of shock waves seems to depend on multiple-factor interactions, which vary depending on the type of bacteria.

\section{Contributed Papers}

\section{0:40}

3aBB6. Numerical simulation of shock and bubble dynamics in shockwave lithotripsy. Tim Colonius and Michel Tanguay (California Inst. of Technol., Pasadena, CA 91125, colonius@ caltech.edu)

Theoretical evaluation of the efficacy of stone comminution (and potential for tissue damage) during shockwave lithotripsy requires knowledge of the complex stress fields associated with both the incident focussing shock and the dynamics of cavitation bubbles that it induces. While simple models from geometrical acoustics and subsequent modeling of spherical bubbles in isolation (Gilmore equation) can provide estimates, high-speed photography in vitro reveals a far more complex flow with bubble number densities that are sufficiently high such that collective effects associated with a cloud of bubbles are important. This talk will describe a modeling effort aimed at estimating stresses from these complex lithotripter generated flow fields. We compute the time-dependent, compressible, ensemble-averaged two-phase flow equations with a finitedifference scheme. Detailed modeling of the dynamics of bubbles (on the microscale) and high-order weighted essentially nonoscillatory shockcapturing schemes are employed. The model is compared to hydrophone and passive cavitation detection measurements, as well as qualitative comparison with high-speed photography. Finally, we explore collective bubble mechanisms ranging from defocusing and shielding of the stone (for high bubble densities in the focal region) to enhanced stresses due to concerted cloud collapse in a dual-pulse lithotripsy configuration. [Work supported by NIH P01 DK-43881 and NSF under grant CTS-9979258.]

\section{0:55}

3aBB7. Dynamical response of a bubble submitted to two following shock waves. Marie-Caroline Jullien (Faculty of Appl. Phys., Univ. of Twente, WB Bldg., P.O. Box 217, 7500 AE Enschede, The Netherlands)

A numerical study of the dynamical response of a bubble submitted to two following shock waves is reported. After the passage of a shock wave, a micron-size bubble expands enormously, reaching millimeter size, and then inertially collapses; this is the so-called cavitation phenomenon. The influence of the passage of a second shock wave on the bubble inertial collapse control is investigated. This control may have a considerable impact for drug delivery or gene transfer, by the use of extracorporeal shock wave lithotripsy, which is a technique already used in medical treatment for kidney stones. It is shown that the dynamical response of the bubble qualitatively depends strongly on the forcing shape. Furthermore, for a given forcing shape, the dynamical response depends on both the delay and forcing amplitude ratio between the two applied shock waves. [This work has been done in collaboration with Ruediger Toegel, ClausDieter Ohl, and Detlef Lohse (Twente University).]

\section{1:10}

3aBB8. Modeling the pressure pulse shape of piezoelectric lithotripters. Thomas Dreyer and Rainer Riedlinger (Universitaet Karlsruhe, IHE-Akustik, Kaiserstr. 12, D-76128 Karlsruhe, Germany, Thomas.Dreyer@ihe.uka.de)

Piezoelectric focusing transducers are widely used in extracorporeal lithotripsy. To optimize the therapeutically relevant focal pressure pulse it is necessary to affect the generated pulse shape at the transducer surface. Therefore a modeling approach is required containing the acoustomechanical properties of the transducer structure as well as the influence of the electrical drive. The procedure presented here uses three dimensional transient finite element simulations to calculate an electro-acoustical impulse response of the transducer structure and linear systems theory to model the influence of the driving circuit on the emitted acoustical signal. Applying a short electrical pulse an acoustical impulse response can be simulated under plane wave conditions, which is valid at sufficiently large distances from the transducer. Focal pressures are estimated rapidly by linear calculations or accurately by a nonlinear propagation model. The influence of electrical drive conditions on the emitted acoustical signal is investigated very efficiently by a convolution with the desired electrical input, avoiding FEM simulations for each case. Reverting this process the 
required driving voltage course for a given pressure signal is determined. Alterations of the pressure signal in terms of pulse width and tensile components are demonstrated theoretically, varying the design parameters of the transducer.

\section{$11: 25$}

3aBB9. Comparison of the cavitation fields of an electromagnetic and electrohydraulic lithotripter. Parag Chitnis and Robin Cleveland (Aerosp. and Mech. Eng. Dept., Boston Univ., 110 Cummington St., Boston, MA 02215)

We contrast the cavitation of two lithotripters of different shock wave (SW) generation: electrohydraulic (EHL) and electromagnetic (EML). The cavitation field induced by the SWs was measured using a dual passive cavitation detector consisting of two $1 \mathrm{MHz}$ focused transducers. The characteristic time $\left(t_{c}\right)$ was obtained from the time difference of the arrival of the SW and the inertial collapse of the cavitating bubble. The $t_{c}$ increased significantly with energy level for both lithotripters. At the highest energy level, $t_{c}=332 \mu \mathrm{s} \pm 65 \mu \mathrm{s}$ for the EML and $t_{c}=277 \mu \mathrm{s}$ $\pm 43 \mu \mathrm{s}$ for the EHL. Increasing the pulse rate frequency from $1 \mathrm{~Hz}$ to 2 $\mathrm{Hz}$ led to a significant increase in $t_{c}$ for the EHL but no significant change for the EML. The region of strong cavitation activity corresponded with the focal region of the lithotripters. Based on a $6 \mathrm{~dB}$ decrease in the amplitude of the inertial collapse signal, the region of strong cavitation was $7 \mathrm{~mm}$ wide for the EHL and $2 \mathrm{~mm}$ wide for the EML. Measurements of the acoustic pressure indicate that the $-6 \mathrm{~dB}$ width of the focal spots were $12 \mathrm{~mm}$ and $4 \mathrm{~mm}$, respectively. [Work supported by the NIH P01DK43881.]

WEDNESDAY MORNING, 4 DECEMBER 2002

CORAL SEA 1 AND 2, 8:30 TO 11:20 A.M.

\title{
Session 3aMU
}

\section{Musical Acoustics: Wind Instrument Measurement Techniques}

\author{
Peter L. Hoekje, Cochair \\ Department of Physics and Astronomy, Baldwin-Wallace College, 275 Eastland Road, Berea, Ohio 44017 \\ Leonardo Fuks, Cochair \\ Escola de Musica, Universidade do Brazil-UFRJ, Rua do Passeio 98, Rio de Janeiro 20021-290, Brazil
}

Chair's Introduction-8:30

Invited Papers

8:35

3aMU1. From air to rubber: New techniques for measuring and replicating mouthpieces, bocals, and bores. Leonardo Fuks (Escola de Musica, Universidade do Brasil-UFRJ, Rua do Passeio 98, Rio de Janeiro 20021-290, Brazil)

The history of musical instruments comprises a long genealogy of models and prototypes that results from a combination of copying existing specimens with the change in constructive parameters, and the addition of new devices. In making wind instruments, several techniques have been traditionally employed for extracting the external and internal dimensions of toneholes, air columns, bells, and mouthpieces. In the twentieth century, methods such as pulse reflectometry, x-ray, magnetic resonance, and ultrasound imaging have been made available for bore measurement. Advantages and drawbacks of the existing methods are discussed and a new method is presented that makes use of the injection and coating of silicon rubber, for accurate molding of the instrument. This technique is harmless to all traditional materials, being indicated also for measurements of historical instruments. The paper presents dimensional data obtained from clarinet and saxophone mouthpieces. A set of replicas of top quality clarinet and saxophone mouthpieces, trombone bocals, and flute headjoints is shown, with comparative acoustical and performance analyses. The application of such techniques for historical and modern instrument analysis, restoration, and manufacturing is proposed.

9:05

3aMU2. Measurement of the acoustic response of a wind instrument with application to bore reconstruction. Maarten van Walstijn and Murray Campbell (Dept. of Physics and Astronomy, Univ. of Edinburgh, Mayfield Rd., Edinburgh EH9 3JZ, UK)

Reconstruction of a bore from measured acoustic response data has been shown to be very useful in studying wind instruments. Such data may be obtained in different ways; directly measuring the frequency-domain response of an acoustic bore has some distinct advantages over directly measuring time-domain data (for example, by pulse reflectometry), but so far has been unsuitable for producing input data for deterministic bore reconstruction algorithms, due to the limited accuracy at high frequencies. In this paper a method is presented for large-bandwidth measurement of the input impedance of a wind instrument using a cylindrical measurement head with multiple wall-mounted microphones. The influence of the number of microphones and the types of calibration impedance on the accuracy will be discussed, and bore reconstructions derived using this technique will be compared with reconstructions obtained using pulse reflectometry. [Work supported by EPSRC.] 
3aMU3. Impedance comparison of high-quality alto and soprano saxophones. Vincent Gibiat (LAMI Univ. Paul Sabatier, 118 route de Narbonne, 31062 Toulouse Cedex, France) and Jerome Selmer (Henri Selmer, Paris, France)

Measuring the input impedance of high-quality wind instruments requires both a system able to provide the physical quantity in less than a few seconds and the possibility to do the measurement on a wide number of instruments. If we have such a system measuring system giving us the possibility to obtain impedance curves for the whole musical range of the instrument the second condition cannot be achieved without the strong collaboration of an instrument maker. The present study has been realized on Selmer saxophones and on instrmuents from two other factories. They include vintage instruments (as Mark VI) and the most recent commercialized ones. It allows one to study the evolution of the instruments. We will show that some alto saxophone models have reached an incredible harmonicity of their resonances while less common models present other characteristics. We will discuss these results and their relations with the audible quality of the instruments.

\section{0:05}

3aMU4. Measuring acoustic impedances using a semi-infinite waveguide reference: Applications to wind instruments and vocal tracts. Joe Wolfe, John Smith, John Tann, and Ryan France (School of Phys., Univ. of New South Wales, Sydney 2052, Australia)

Acoustic pressures may generally be measured with much greater sensitivity, dynamic range, and frequency response than acoustic currents. Consequently, most measurements of acoustic impedance consist of comparison with standard impedances. The method reported here uses a semi-infinite waveguide as the reference because its impedance is purely resistive, frequency independent and accurately known, independent of theories of the boundary layer. Waveguides are effectively infinite for pulses shorter than the echo return time, or if the attenuation due to wall losses (typically $80 \mathrm{~dB}$ ) exceeds the dynamic range of the experiment. The measurement signal from a high output impedance source is calibrated to have Fourier components proportional to $f n$, where $\mathrm{n}$ may be 1 for convenience or chosen to improve the signal:noise ratio. The method has been used on diverse systems over the range $50 \mathrm{~Hz}$ to 13 $\mathrm{kHz}$. When applied to systems with simple geometries, the technique yields results with a little higher wall losses than those expected from the calculations of Rayleigh and Benade. Discontinuities introduce further losses as well as the expected departures from simple one-dimensional models. Measurements on musical wind instruments and on the human vocal tract are reported. [Work supported by the Australian Research Council.]

\section{Contributed Papers}

\section{$10: 35$}

3aMU5. Acoustic input impedance measurements on brass instruments. Robert W. Pyle, Jr. (11 Holworthy Pl., Cambridge, MA 02138, rpyle@post.harvard.edu)

Measurement of the acoustic input impedance of a brass instrument can reveal something about the instrument's intonation, its reasonable playing range, its tone color, and perhaps whether the mouthpiece used for the impedance measurement is appropriate for the instrument. Such measurements are made at sound-presssure levels much lower than those encountered under playing conditions. Thus, impedance measurements may offer the only feasible way to infer something about the playing characteristics of instruments, typically museum specimens, that are too rare or too fragile to be played. In this paper the effects of some of the available choices of sound source and stimulus signal on measurement accuracy will be explored. Driver-transducer nonlinearity, source impedance, signal-tonoise ratio, and any necessary signal processing will be discussed.

\section{0:50}

3aMU6. Pulse reflectometry as an acoustical inverse problem: Regularization of the bore reconstruction. Barbara J. Forbes, David B. Sharp (Dept. of Environ. and Mech. Eng., The Open Univ., Walton Hall, Milton Keynes MK7 6AA, UK, b.forbes@open.ac.uk), and Jonathan A. Kemp (Univ. of Edinburgh, Edinburgh EH9 3JZ, UK)

The theoretical basis of acoustic pulse reflectometry, a noninvasive method for the reconstruction of an acoustical duct from the reflections measured in response to an input pulse, is reviewed in terms of the inversion of the central Fredholm equation. It is known that this is an ill-posed problem in the context of finite-bandwidth experimental signals. Recent work by the authors has proposed the truncated singular value decomposition (TSVD) in the regularization of the transient input impulse re- sponse, a non-measurable quantity from which the spatial bore reconstruction is derived. In the present paper we further emphasize the relevance of the singular system framework to reflectometry applications, examining for the first time the transient bases of the system. In particular, by varying the truncation point for increasing condition numbers of the system matrix, it is found that the effects of out-of-bandwidth singular functions on the bore reconstruction can be systematically studied.

\section{1:05}

3aMU7. Body vibrational spectra of metal flute models. Clare M. Hurtgen (Jaffe Holden Acoustics, Inc., 114A Washington St., Norwalk, CT 06854, churtgen@jhacoustics.com) and Dewey T. Lawson (Duke Univ., Durham, NC 27708)

For years, flutists have argued over the tonal advantages of using different precious metals for their instruments. Occasionally, scientists have entered the fray and attempted to offer an objective point of view based on experimental measurements. However, their research often involved actual instruments and performers, ignoring variations in wall thickness, craftsmanship, and human consistency. These experiments have been conducted using a variety of methods; all have concluded that wall material has no effect on tone. This paper approaches the question using simple tubular models, excited by a wind source through a fipple mouthpiece. The amplitude and phase of the harmonic components of the body vibrational signal were measured with a stereo cartridge. Results demonstrated the existence of complex patterns of wall vibrations in the vicinity of a tone hole lattice, at frequencies that match significant harmonics of the air column. Additionally, the tube wall was found to expand in a nonuniform or "elliptical" manner due to the asymmetry of the tone holes. While this method is somewhat removed from direct musical applications, it can provide an objective, quantitative basis for assessing the source of differences among flutes. [Work financed by two Undergraduate Research Support grants from Duke University.] 


\title{
Session 3aNS
}

\section{Noise: Hearing Protection I}

\author{
Elliott H. Berger, Cochair \\ EAR/Aearo Company, 7911 Zionsville Road, Indianapolis, Indiana 46268-1657 \\ Daniel P. Salomon, Cochair \\ Comaudi, Patriotismo 706, Colonia Mixcoac 03730, D.F. Mexico
}

Chair's Introduction-8:30

Invited Papers

8:35

3aNS1. International hearing protector standardization. Torben Poulsen (Acoust. Technol., Oersted-DTU, Bldg. 352, DTU, DK-2800 Lyngby, Denmark, tp@oersted.dtu.dk)

Hearing protectors shall fulfill some minimum requirements to their performance. As hearing protector manufacturers sell the products all over the world, the testing and certification of hearing protectors has become an international issue. The ISO working group WG17 under the headlines Acoustics, Noise, produce hearing protector standards to be used at an international level. The presentation will cover the ongoing work in WG17, including the revision of existing standards (ISO 4869-1, ISO 4869-3), upcoming new standards (ISO 4869-7) and the plans and status for future standards (performance in impulse noise, protectors with active noise reduction). Furthermore, an overview of the present European standards (CEN) and the relation to American and Australian/New Zealand standards will be discussed.

\section{8:55}

3aNS2. Development of Mexican standard on hearing protection devices. Daniel P. Salomon and Jose N. Razo (Comaudi, Patriotismo 706, Colonia Mixcoac, 03730, Mexico D. F., gerencia@comaudi.com)

A working group was established by the Secretaria del Trabajo (similar to OSHA) to work on a standard whose objective was to protect workers from noise by trying to predict the performance of HPDs as personal protection equipment in the workplace. Various standards were analyzed including ISO4869, AS-NZ 1270, and ANSI. All evaluate attenuation as a performance descriptor. Experimenter-Fit methods tend to overrate field performance due to very different field versus laboratory conditions. Subject-Fit methods result in lower attenuation values which are more likely to be achieved by groups of workers, even if inadequately motivated and trained. Subject-Fit methods encountered large resistance, including laboratories' difficulties in finding naive subjects, commercial interests of manufacturers, risk of overprotection, etc. Having settled for an Experimenter-Fit method, the intent is to do more to help protect workers' hearing through other means available. A thorough, yet fairly simple, HPD Selection and Usage Guide was prepared as an Informative Appendix. Relevant information should be provided to the user with the Packaging. NRR wars should be avoided. The standard includes a list of contents, instructions, and warnings that must be included. A sample label containing key information is detailed in another Informative Appendix.

\section{9:15}

3aNS3. The subjective testing of hearing protectors and issues of interlaboratory variability-The Australian/New Zealand experience. Warwick Williams (Natl. Acoust. Labs., 126 Greville St., Chatswood, NSW Australia, warwick.williams@hearing.com.au)

Over the past few years the proposal to use "inexperienced" subjects to test the attenuation of hearing protectors has been controversial, particularly in the USA and in connection with the development of draft ISO 4869-7 Acoustics-hearing protectorsPart 7: Subjective method for measurement of sound attenuation-Subject-fit method. Much of the discussion centers around the belief that by using "inexperienced," "unassisted" test subjects attenuation results will be unrepresentative of the "true" figure. Both Australia and New Zeland have been using subjective testing of hearing protectors with inexperienced, unassisted test subjects for many years. The results have been positive and the conjectured difficulties have not eventuated. Any "unusual" results have been readily explained. The Australian acoustic community has no difficulty with the test methodology. Further it is the opinion of Occupational Health and Safety workers that "inexperienced," subjective testing most closely represents attenuation values typical in the well informed workplace. 
3aNS4. Hearing protection: Limits to attenuation. Elliott H. Berger, Ronald W. Kieper (E-A-R/Aearo Co., 7911 Zionsville Rd., Indianapolis, IN 46268-1657), and Dan Gauger (Bose Corp., The Mountain, Framingham, MA 01701-9168)

With louder and louder weapon systems being developed and military personnel being exposed to steady noise levels approaching and sometimes exceeding $150 \mathrm{~dB}$, the interest in greater amounts of protection is evident. When the need for communications is included in the equation, the situation is even more extreme. New initiatives are underway to design improved hearing protection, including active noise reduction (ANR) earplugs and perhaps even cancellation of head-borne vibration. With that in mind it may be useful to explore the limits to attenuation, and whether they can be approached with existing technology. Data on the noise reduction achievable with high-attenuation foam earplugs, as a function of insertion depth, will be reported. Previous studies will be reviewed that provide indications of the bone-conduction (BC) limits to attenuation that, in terms of mean values, range from 40-60 dB across the frequencies from $125 \mathrm{~Hz}$ to $8 \mathrm{kHz}$. Additionally, new research on the effects of a flight helmet on the BC limits, as well as the potential attenuation from deeply inserted passive foam earplugs or communication earplugs, worn with passive earmuffs, or with active-noise reduction (ANR) earmuffs, will be also examined.

9:55

3aNS5. Do sound restoration hearing protectors provide adequate attenuation for gunfire noise? John R. Franks and William J. Murphy (NIOSH Hearing Loss Prevention Section, 4676 Columbia Pkwy., M.S. C-27, Cincinnati, OH 45226-1998, jrf3@cdc.gov)

Sound restoration earmuffs have an electronics package installed in a passive earmuff shell or earplug body. The electronics package consists of a microphone placed on the outside of the ear cup or earplug and a limiting amplifier driving a loudspeaker placed inside the ear cup or the earplug. The devices are designed to provide unity gain or better to low-level signals and to shut off when sound exceeds a given level. The effects of several sound restoration earmuffs and two nonlinear orifice earplugs on gunfire for several different weapon types were analyzed. In general, the nature of the electronics packages was such that the studied devices provided the same attenuation when turned on as when turned off. However, as with passive devices, a single protector, be it by earplug or earmuff, appears to be inadequate for gunfire when more than just a few shots are fired. Sound restoration earmuffs are best used with a well-fitted earplug, since the electronics can compensate for both the insertion loss of the earmuff and the earplug. However, nonlinear orifice earplugs may not provide sufficient protection for extended sessions of target practice.

10:15-10:30 Break

10:30

3aNS6. Industrial warning signal detection under augmented hearing protection devices. John G. Casali and Gary S. Robinson (Dept. of Industrial \& Systems Eng., Auditory Systems Lab., Virginia Tech, 260 Durham Hall, Blacksburg, VA 24061, jcasali@ vt.edu)

The results from several experiments concerning the effects of passive, Amplitude-Sensitive Sound Transmission (ASST), and Active Noise Reduction (ANR) HPDs on vehicle backup alarm detection by normal hearers are reviewed. Masked thresholds under a Peltor electronic ASST earmuff and an AEARO orifice-based ASST earmuff were found not to differ significantly from thresholds under conventional earmuffs of similar volume to the ASST devices, in pink noise of 75,85 , and $95 \mathrm{dBA}$. A separate analysis using a KEMAR manikin indicated that subjects' chosen settings of the Peltor ASST earmuff gain control did not significantly increase the noise exposure dose over the earmuff's amplifier-off setting. In low frequency-biased "red" noise conditions (averaged across 85 and $100 \mathrm{dBA}$ ), masked thresholds under a Bose ANR earmuff and an AEARO foam earplug were significantly lower (better) than those under a Peltor passive earmuff having weaker low frequency attenuation, but there were no differences among the 3 devices in pink noise, nor at $85 \mathrm{dBA}$ in either noise. Detection thresholds in $85 \mathrm{dBA}$ noise were 3.2-4.5 dB lower in the 3 protected conditions than in the unprotected condition, attesting to the fact that HPD use can facilitate signal detection under certain conditions for normal hearers. [Work supported by U.S. Bureau of Mines (now NIOSH), Bose Corp., Aearo Corp.]

\section{0:50}

3aNS7. Single number to represent hearing protector attenuation. Samir N. Y. Gerges, Eric N. Gaste (Mech. Eng. Dept., Federal Univ. of Santa Catarina, Cx.P. 476, Florianopolis, SC, Brazil, CEP: 88040-900), and Vera M. Steffen (Federal Univ. of Rio rand de sul, Porto Alere, RS, Brazil)

The existing single numbers for hearing protector noise attenuation are NRR, SNR, HML, and NRRsf. All of these numbers are calculated using a standard ambient noise called pink noise with a total level of $100 \mathrm{dBC}$. This pink noise does not represent real industrial noise. This pink noise has its peak values in $\mathrm{dBA}$ at $2 \mathrm{kHz}$, which is not usually the case for most real industrial environments. Also, this single number is not associated with statistical distribution and standard deviation. In this paper a trial is presented to calculate the NRRsf using measured industrial noise spectrum. The calculation is carried out for a typical foam-hearing protector. The REAT naive subject results obtained from laboratory measurements of 20 subjects using ANSI 12.6-1997 (B) gives 20 individual attenuations as function of frequency. For each one of the real noise spectra measured in industry, using one subject attenuation, an NRRsf individual number is obtained. The average NRRsf and standard deviation of these attenuation values are presented along with their statistical distribution. These results are discussed and compared to the single classical numbers. 
3aNS8. Issues regarding hearing protection device use in manufacturing and mining. Thais Morata (Hearing Loss Prevention Section, NIOSH C27/4676, Columbia Pkwy., Cincinnati, OH 45226)

In this study reasons offered by workers for not consistently using hearing protectors are examined. Study groups were comprised of: (1) 124 workers exposed to various levels of noise in a manufacturing company, located in Sao Paulo, Brazil. Data on work history, psychosocial aspects of the workers job, medical history, present health, stress, occupational and nonoccupational exposures to noise or chemicals, and lifestyle factors were collected through interviews. The participants had their hearing and noise exposure assessed. Sixty-four percent of the workers indicated that they wore hearing protectors, but only 20 percent of that subgroup stated that they wore the devices all the time when noise-exposed. (2) Forty-four workers and supervisors from the manufacturing and mining sectors in Cincinnati and Pittsburgh. Participants were interviewed or took part in focus groups discussions on a variety of hearing conservation topics, including problems with the use of HPDs. The variables significantly associated with a workers' decision not to wear hearing protectors consistently included interference with communication, interference with job performance, comfort issues, selfperception of hearing condition, and inability to detect warning signals when wearing HPDs. These are issues to be addressed in order to promote a more effective use of HPDs.

\title{
Contributed Papers
}

$11: 30$

3aNS9. Software development for NIOSH hearing protector testing. William J. Murphy, John R. Franks (NIOSH Hearing Loss Prevention Section, 4676 Columbia Pkwy., M.S. C-27, Cincinnati, OH 45226-1998, wjm4@cdc.gov), and Douglas T. Rohrer (DTR Software Design and Development, Cincinnati, OH 45226)

In June 1998, NIOSH researchers recognized the necessity of rebuilding the NIOSH Hearing Protector Device Testing Laboratory. Several design requirements influenced the software development and hardware selection. First, the hardware must be commercially available and easily operated from personal computers that use the Microsoft Windows operating system. Second, software must have the flexibility to support not only the demands of a research laboratory and field studies but also be configurable such that a commercial laboratory could use the software for production testing of protectors. Finally, the complete package must ensure the use of standard operating procedures for the purpose of achieving and maintaining NVLAP certification for the ANSI S12.6 and S3.19 standards for the measurement of real-ear attenuation at threshold. The total system has been operational since May 2002 and has been installed in three laboratories to date. This paper will present how these competing needs were addressed in the development of the NIOSH HPD Lab software.

\section{1:45}

3aNS10. Sound propagation in straight, conical, exponential and real external ear canal. Alexsander Fortkamp, Washington J. N. de Lima, and Samir N. Y. Gerges (Federal Univ. of Santa Catarina, Cx.P. 476, Florianopolis, SC, Brazil)

Mathematical and numerical modeling of the noise attenuation of hearing protectors (HP) is a powerful tool for optimizing the HP design. The human ear canal has a complex geometry and therefore can be modeled by numerical methods. Below the cut-off frequency, plane wave propagation can be modeled by simple geometric. In this paper straight, conical, exponential, and real ear canal geometry are considered. The Webster equation is used to model the sound propagation inside these canals. In this paper a comparison of sound field propagation obtained from a plane wave model of the simple geometries and the finite elements model of the real ear are presented.

WEDNESDAY MORNING, 4 DECEMBER 2002

CORAL ISLAND 1 AND 2, 8:30 TO 11:45 A.M.

\section{Session 3aPAa}

\section{Physical Acoustics and Signal Processing in Acoustics: The Coda and Other Stochastic Seismic Signals I}

\author{
Richard L. Weaver, Chair \\ Department of Theoretical and Applied Mechanics, University of Illinois, 216 Talbot Laboratory, 104 South Wright Street, \\ Urbana, Illinois 61801
}

\section{Invited Papers}

\author{
8:30
}

3aPAa1. Seismic coda. Michael Fehler (Los Alamos Natl. Lab., M.S. D443, Los Alamos, NM 87545)

Observations and analysis of seismic scattering in the heterogeneous earth have grown from initial observations in the 1960s into a well-developed subfield of seismology. The field presents many challenging, interesting, and fruitful areas of research for seismologists. The term seismic coda has been used to describe the overall discipline but the term coda was originally used only for the tail portion of the seismograms recorded within $100 \mathrm{~km}$ of earthquakes. Several characteristics of seismic coda have been identified that provide important constraints on models to explain the observed waveforms. It is now generally accepted that coda is a superposition of waves that have been multiply scattered from heterogeneities in the Earth's lithosphere. Coda waves, even if they are not completely understood, have practical use for seismologists because some of their characteristics lead to extremely reliable methods for quantifying relative sizes of earthquakes and relative local site amplification characteristics in seismic hazards investigations. Observations and theoretical modeling studies of coda waves have provided insight into the complexity of wave propagation in the Earth and have yielded new insights into the character of the Earth's lithosphere. 
Radiative transport equations for elastic waves in random media can be derived from first principles in a certain regime of physical parameters. They account for both longitudinal and transverse waves, as well as polarization. Transport boundary conditions can also be derived from first principles. An important application of transport theory is to the analysis of the deep coda of elastic waves, where some rather interesting phenomena of energy distribution emerge. A reference to work in this area is by Ryzhik et al. [Wave Motion 24, 327-370 (1996)]. Another reference, more directly related to seismics, is Ryzhik et al. [Bull. Seismol. Soc. Am. 86, 1107-1115 (1996)].

9:30

3aPAa3. Quasi-2D diffusion of elastic waves. Bart Van Tiggelen, Nicolas Tregoures (Lab. de Physique et Modlisation des Milieux Consenss, CNRS/UJF, Maison des Magistres, BP 166, F-38042 Grenoble, France), Michel Campillo, Ludovic Margerin, and Celine Lacombe (Lab. de Geophysique Interne et Tectonophysique, F-38041 Grenoble, France)

Recent studies on seismic Coda and seismic equipartition have shown the possibility to model the propagation of seismic waves around $5 \mathrm{~Hz}$ in the crust using a heterogeneous layered model with a free surface overlying a homogeneous half-space. The depth of the layer is roughly $30 \mathrm{~km}$. If the mean free path of the waves is in excess of this value, and this is probably the case in many places in the world (except perhaps Mexico), the wave diffusion should be described by mode diffusion in a 2D layer, rather than plane waves in a 3D medium. This notion affects many observed and potentially observable quantities, such as equipartition, leakage of energy, and coherent backscattering. [Work supported by the French Ministry of Research.]

10:00-10:15 Break

10:15

3aPAa4. Long codas of coupled wave systems in seismic basins. Thomas H. Seligman (Centro de Ciencias Fisicas, Univ. of Mexico, Cuernavaca, Mexico)

Quite some time ago it was pointed out that the damage patterns and Fourier spectra of the 1985 earthquake in Mexico City are only compatible with a resonant effect of horizontal waves with the approximate speed of sound waves in water [see Flores et al., Nature 326, 783 (1987)]. In a more recent paper it was pointed out that this indeed will occur with a very specific frequency selection for a coupled system of Raleigh waves at the interface of the bottom of the ancient lakebed with the more solid deposits, and an evanescent sound wave in the mud above [see J. Flores et al., Bull. Seismol. Soc. Am. 89, 14-21 (1999)]. In the present talk we shall go over these arguments again and show that strong reflection at the edges of the lake must occur to account for the strong magnification entailing necessarily a long coda, and that the mecanism can be understood in the same terms.

\section{Contributed Papers}

\section{0:45}

3aPAa5. Numerical modeling of seismogram envelopes in 2-D random media. Michael Fehler (Los Alamos Natl. Lab., M.S. D443, Los Alamos, NM 87545)

Several portions of seismograms recorded from regional earthquakes cannot be easily explained as resulting from waves propagating along deterministic paths within the Earth. For example, seismic coda, which is the tail portion of the seismogram of an earthquake recorded at distances of less than $100 \mathrm{~km}$, is considered as resulting from waves that are multiply scattered from random heterogeneities in the Earth's lithosphere. At greater distances, observations that the duration of the initial arriving wave packet is much longer than the source-time duration is explained as being due to multiple forward scattering along the path between the source and the receiver. To investigate these phenomena, we use a finite difference method to numerically simulate 2-D scalar-waves that propagate through random media characterized by a von Karman autocorrelation function. Such media are considered to be appropriate models for the random component of the structure of the Earth's lithosphere. We investigate the characteristics of the resulting wavefields and compare them with those of observed seismograms.

\section{1:00}

3aPAa6. Anisotropic diffusion of elastic waves. Joseph A. Turner and Liyong Yang (Dept. of Eng. Mech., W317.4 NH, Univ. of Nebraska-Lincoln, Lincoln, NE 68588, jaturner@unl.edu)

The scattering of elastic waves in heterogeneous, anisotropic media is relevant for ultrasonic and seismic waves for media including textured polycrystalline media, fiber-reinforced composites, and media containing oriented cracks. Elastic waves propagating through such media lose en- ergy due to scattering from the heterogeneities of the material. In anisotropic media, this scattering attenuation is a function of propagation direction. Here, the case of transverse isotropy is examined. The problem is formulated in terms of the Dyson and Bethe-Salpeter equations for the mean and covariance of the anisotropic Green's function, respectively. Solution of the Dyson equation, using the first-order smoothing approximation (FOSA), gives attenuations for shear horizontal, quasicompressional, and quasishear waves as a function of direction and frequency. The FOSA is also used to reduce the Bethe-Salpeter equation to an elastic radiative transfer equation. In the limit of many scattering events, this equation then reduces to an anisotropic diffusion equation. The primary result is the diffusion tensor given in terms of the various scattering functions that characterize the transfer of elastic energy between the different wave types of directions. The results are discussed in terms of applications to textured metals and media with aligned cracks. [Work supported by NSF and DOE.]

\section{1:15}

3aPAa7. Diffusion of ultrasonic waves in porous glass bead sinters. John H. Page, James Beck, Russel Holmes, and Jake Bobowski (Dept. of Phys. and Astron., Univ. of Manitoba, Winnipeg, MB R3T 2N2, Canada)

Sintered networks of glass beads form an interesting example of a porous medium, analogous to a very porous rock, in which very strong multiple scattering of elastic waves is observed when the ultrasonic wavelength is comparable with the size of the pores. To investigate the diffusive transport of energy by multiply scattered waves, the transmission of the diffuse energy flux through finite slabs of this material has been measured. From these data, the diffusion coefficient $D$, as well as the absorption time, was determined by fitting the predictions of the diffusion approximation to the experimental time-of-flight profiles. To accurately measure 
$D$, the internal reflection of diffuse waves at the sample boundaries was taken into account by extending the method used previously for acoustic waves [J. H. Page et al., Phys. Rev. E 52, 3106 (1995)]. The frequency dependence of the diffusion coefficient was measured over an extended range of frequencies, and compared with estimates of $D$ from ballistic measurements of the scattering mean free path and group velocity. Because of its relatively simple structure, this material may be an ideal system for probing the diffusion of elastic waves, where diffuse waves have mixed character consisting of both longitudinal and transverse polarizations.

\section{1:30}

3aPAa8. The coda of artificial shots on volcanos. Ulrich Wegler (Institut für Geophysik und Geologie, Universität Leipzig, Talstraße 35, 04103 Leipzig, Germany, uli@rz.uni-leipzig.de)

The data of active seismic experiments at Merapi volcano and at Vesuvius volcano are used to analyze the scattering of elastic waves in the shallow structure of these volcanos. The seismograms from artificial explosions located on the volcanos are characterized by spindle-like envelopes, small or missing $P$-onsets, missing $S$-onsets, and long codas. These unusual shapes of the envelopes can be explained by strong multiple scattering in the shallow heterogeneous eruptive material and are modeled using the diffusion model. As a result diffusivities of $d=0.05 \mathrm{~km}^{2} / \mathrm{s}$ in the case of Merapi and of $d=0.1 \mathrm{~km}^{2} / \mathrm{s}$ in the case of Vesuvius are obtained independent of frequency between 2 to $20 \mathrm{~Hz}$. Assuming the dominance of shear waves in the coda and a typical $S$-wave velocity of around $1.5 \mathrm{~km} / \mathrm{s}$ for the shallow volcano this corresponds to a transport mean free path of only $100 \mathrm{~m}$ in the case of Merapi and of only $200 \mathrm{~m}$ in the case of Vesuvius. The corresponding length scales for intrinsic attenuation, on the contrary, depend on frequency and are at least one order of magnitude larger than the transport mean free path. These results show that multiple scattering is an important effect for the seismic wave propagation in volcanic environments.

WEDNESDAY MORNING, 4 DECEMBER 2002

CORAL GALLERY FOYER, 10:00 A.M. TO 12:00 NOON

\title{
Session 3aPAb
}

Physical Acoustics: Thermoacoustics and Resonators

\author{
Matthew E. Poese, Cochair \\ Graduate Program in Acoustics, Pennsylvania State University, P.O. Box 30, State College, Pennsylvania 16804 \\ Guadalupe Huelsz, Cochair \\ Centro de Investigacion en Energia, A. P. 34, Temixco, Morelos 62580, Mexico
}

\section{Contributed Papers}

\section{0:00}

3aPAb1. Simulations of acoustic streaming inside and surrounding thermoacoustic stacks. Mark F. Hamilton, Yurii A. Ilinskii, and Evgenia A. Zabolotskaya (Dept. of Mech. Eng., Univ. of Texas, Austin, TX 78712-1063)

Acoustic streaming generated inside and surrounding thermoacoustic engine stacks by standing waves in resonators is investigated numerically. The study is performed using the nonlinear 2-D code described recently by the authors [J. Acoust. Soc. Am. 111, 2076 (2002)]. The stack consists of parallel plates of negligible thickness. There is no restriction on plate separation, or on the length or position of the stack in the resonator. Temperature dependence of the fluid viscosity is taken into account. A temperature gradient imposed on the stack drives the sound field. Acoustic streaming patterns, investigated in terms of average mass flow velocity, are calculated by time averaging the momentum density. For typical plate separations on the order of the thermoviscous penetration depth, only the inner, boundary-layer streaming vortices exist between the plates. These inner vortices rotate in directions opposite those of the outer, more familiar Rayleigh streaming vortices encountered in wide channels (wider than about 10 penetration depths). The inner vortices extend beyond the ends of the stack out to distances on the order of the acoustic particle displacement. Flow patterns near the entrances to the stack are investigated as functions of channel width and acoustic amplitude [Work supported by ONR.]
10:15

3aPAb2. Use of complex frequency to analyze thermoacoustic engines. James E. Parker, Mark F. Hamilton (Appl. Res. Labs., Univ. of Texas, Austin, TX 78713-8029), Yurii A. Ilinskii, and Evgenia A. Zabolotskaya (Univ. of Texas, Austin, TX 78712-1063)

Rotts equations of thermoacoustics are expressed in the frequency domain, and as such they describe steady-state conditions. In numerical algorithms such as DELTAE the frequency is considered real, and the solutions correspond to the equilibrium states associated with the critical temperature gradient in the stack. An alternative approach, and the one described here, is to allow frequency to be complex and to determine its value for a specified temperature gradient. A nonzero imaginary part of the frequency indicates a nonequilibrium state, and depending on its sign it determines the rate at which the amplitude of the sound field either increases or decreases initially with time toward equilibrium at the critical temperature gradient. For solutions corresponding to thermoacoustic instability the imaginary part may be interpreted as a negative loss coefficient, which provides an estimate of the nonlinear loss required to offset the instability. This in turn determines the acoustic pressure at saturation. Graphs of the imaginary part versus the position and length of the stack for various resonators provide insight into favorable operating configurations. Consideration is also given to nonconstant temperature gradients along the length of the stack. [Work supported by ONR.] 
10:30

3aPAb3. Performance of a small, low-lift regenerator-based thermoacoustic refrigerator. Matthew E. Poese and Steven L. Garrett (Grad. Prog. in Acoust., P.O. Box 30, State College, PA 16804, poese@psu.edu)

A regenerator-based thermoacoustic refrigerator [Swift, Gardner, and Backhaus, "Acoustic recovery of lost power in pulse tube refrigerators," J. Acoust. Soc. Am. 105(2), 711 (1999)] has been constructed. It is capable of moving about $5 \mathrm{~W}$ across a $40{ }^{\circ} \mathrm{C}$ temperature span. The machine operates with air at atmospheric pressure and is driven by an off-the-shelf electro-dynamic loudspeaker capable of producing peak-to-mean pressure ratios up to $12 \%$. The thermal core of this research device contains an exhaust-side shell and tube heat exchanger (with water as the secondary heat transfer fluid), a regenerator made of 88 annular stainless-steel screens, and a constantan wire electrical heater that applies a measurable heat load to the cold side of the regenerator. An annular latex diaphragm is placed over the cold side of the regenerator to stop time-averaged mass flow through the regenerator and insulate the cold side [Gedeon, "DC Gas Flows in Stirling and Pulse Tube Cryocoolers," in Cryocoolers 9, edited by R. G. Ross (Plenum, New York, 1997)]. Detailed measurements of heat load, temperature span, and exhaust heat flux will be presented and compared to DeltaE. [Work supported by ONR.]

\section{0:45}

3aPAb4. Competition between two different resonance acoustic modes in a thermoacoustic prime mover. W. R. Canul-Vargas, I. VeraManrique, R. Miranda, Y. Nahmad-Molinari, J. C. Ruiz-Suárez (Departamento de Física Aplicada, Cinvestav-Mérida, Mérida Yucatán 97310, Mexico), and G. Huelsz (UNAM, Temixco, Mor. 62580, Mexico)

When a stack of plates is placed near the closed end of an open tube, and a temperature gradient is applied to the stack (the hotter part next to the sealed tube end), a sound wave is produced. The greater the temperature gradient beyond a critical value, the stronger the sound wave produced. Inversely, to switch off the sound wave one has to decrease the temperature gradient applied to the stack. In the present work we report a new method to turn off and on this thermoacoustic prime mover almost instantaneously, without modifying the temperature gradient. A second resonator of different length and much smaller cross section (a tube with both ends open) is introduced into the first tube until contact with the stack is made. When this new resonator is left in place there is no change in the sound wave, but as soon as the end of the small tube outside the main resonator is closed, the sound wave disappears completely. Under this condition, the system goes rapidly from an acoustic thermodynamic cycle in which heat is transported by the wave, to a simple heat diffusion through the stack. We study the physics of this intriguing phenomenon.

\section{1:00}

3aPAb5. Measurement of the time evolution of Rayleigh streaming in high-amplitude standing waves. Michael W. Thompson and Anthony A. Atchley (Grad. Prog. in Acoust., The Penn State Univ., 217 Appl. Sci. Bldg., University Park, PA 16802, mwt126@psu.edu)

The time evolution of the axial component of the Rayleigh streaming velocity field in a cylindrical, air-filled resonator has been measured using laser Doppler anemometry. At low acoustic amplitudes, the measured field is in agreement with classical theory. The axial component varies sinusoidally along the axial direction and quadratically along the radial direction, and the position of maximum streaming velocity on the axis is located midway between the velocity node and velocity antinode. At higher acoustic amplitudes, the streaming velocity field initially resembles the classical result, but becomes progressively distorted as time passes. The position of maximum streaming velocity on the axis shifts towards the velocity antinode, while the streaming velocity outside the boundary layer, near the resonator wall, remains unaffected. Additionally, the radial dependence of the axial streaming velocity deviates from its initially parabolic shape, becoming flatter near the velocity node and steeper near the velocity an- tinode. The length of time required for the streaming to reach steady state is on the order of several minutes for a fundamental frequency of $310 \mathrm{~Hz}$, a resonator radius of $23 \mathrm{~mm}$, and an antinodal acoustic velocity of $6.0 \mathrm{~m} / \mathrm{s}$ peak. [Work supported by ONR.]

\section{1:15}

3aPAb6. Computational and experimental investigation of minor losses in high amplitude acoustic resonators with varied cross section. Andrew J. Doller (Grad Prog. in Acoust., Penn State Univ., State College, PA 16804), Said Boluriaan, Anthony A. Atchley, and Philip J. Morris (Penn State Univ., University Park, PA 16802)

Minor losses in high amplitude oscillatory flows through changes in resonator cross section are not well understood. The application of numerical simulation in the study of these losses has shown promise over the past few years. A computational model has been developed based on a time accurate numerical simulation of the full Navier-Stokes equations on parallel computers. A rectangular clustered mesh is used to capture flow field details in the boundary layers and in the vicinity of changes in cross section. Results of simulations are compared to measurements of acoustic and time-averaged pressures at multiple points along the length of resonators and are compared at multiple drive amplitudes. The resonators consist of two sections of straight brass pipe of different diameters and joined through either steplike or conical couplers. They are driven at the end of the large diameter pipe with a rigid piston. The end of the small tube is rigidly terminated. [Research supported by ONR.]

\section{$11: 30$}

3aPAb7. Effect of mean fluid flow on an acoustic standing wave in an open cavity. Michael Anderson, Kenneth Line, and Ralph Budwig (Dept. of Mech. Eng., Univ. of Idaho, Eng. Phys. Bldg., 324K, Univ. of Idaho, Moscow, ID 83844-0902)

Acoustic radiation pressure can be used to concentrate or remove small particles from an airborne aerosol. In this application, an ultrasonic transducer, mounted flush to one wall of a channel, is used to excite an integer half-wavelength standing wave of high amplitude that propagates perpendicular to the aerosol flow direction. An expression for the Fourier transform of the acoustic pressure in a semi-infinite channel, including the effect of mean fluid flow and finite transducer aperture, has been obtained. A parabolic (laminar) mean flow was assumed. The acoustic pressure was found to be governed by the Mach number of the flow, defined by the projection of the propagation direction relative to the mean flow velocity vector; and the aperture function of the transducer. Near a frequency of 50 $\mathrm{kHz}$, numerical inversions of the acoustic pressure transform showed that the presence of mean flow in the velocity range $0-2 \mathrm{~m} / \mathrm{s}$ caused changes in acoustic pressure on the order of $1 \%-4 \%$. Corresponding experimental measurements showed changes in acoustic pressure up to $10 \%$. The highest changes in measured acoustic pressure were found to occur up- and down stream relative to the transducer, and these patterns were in agreement with predictions of the analytical model.

\section{1:45}

3aPAb8. Feasibility study of acoustic agglomeration of fly ash in shaped resonators. Bart Lipkens and Theodore J. Conrad (Mech. Eng. Dept., Virginia Commonwealth Univ., 601 W. Main St., Richmond, VA 23284-3015)

An interesting method for controlling fine particulate emission is acoustic agglomeration. Acoustic agglomeration is a process where fine particles coalesce and grow into bigger particles when they are subjected to a high intensity sound field. As a result the particle size distribution shifts to larger sizes. Acoustic agglomeration can thus be used as a preconditioner prior to a conventional filter and improve the overall capture rate of the filter. An experimental study was performed to evaluate the potential of a new high intensity acoustics technology, resonant mac- 
rosonic synthesis (RMS), to agglomerate fly ash. RMS allows the generation of very high amplitude shock-free acoustic standing waves within shaped resonators. Experiments were performed in a football shaped resonator at $570 \mathrm{~Hz}$ under varying fly ash loadings and sound pressure levels.
Particle size measurements were obtained using a laser diffraction particlesizing instrument. Reduction of fine particulate matter (diameter of particles less than 5 micron) of up to $50 \%$ has been achieved. [Work supported by Dominion Corp.]

WEDNESDAY MORNING, 4 DECEMBER 2002

CORAL GALLERY FOYER, 8:00 TO 9:45 A.M.

Session 3aPP

\title{
Psychological and Physiological Acoustics: Physiological Acoustics and Cochlear Implants
}

\author{
Linda Polka, Cochair \\ School of Communication Sciences and Disorders, McGill University, Beatty Hall, 1266 Pine Avenue West, Montreal, \\ Quebec H3G 1A8, Canada
}

Fernando Elizondo-Garza, Cochair

Cd Universitaria, P.O. Box 28F, San Nicolas 66450, N.L. Mexico

\section{Contributed Papers}

8:00

3aPP1. The cochlear outer hair cell motility must be based on a tonic change in the membrane voltage: Implications for the cochlear amplifier. Jont B. Allen (Mimosa Acoust., 382 Forest Hill Way, Mountainside, NJ 07092) and Paul F. Fahey (Univ. of Scranton, Scranton, PA 18510)

It has been widely assumed that the function of the $\mathrm{OHC}$ is to increase the sensitivity and frequency selectivity of the cochlea via a phasic $\mathrm{OHC}$ voltage, which controls the soma length. This action is called the cochlear amplifier. According to this view the length of the OHC is assumed to follow the stimulus to the upper frequency limit of hearing, in a phasic manner (cycle by cycle), adding power at the signal frequency. We propose an alternative view that the $\mathrm{OHC}$ controls the dynamic range in a parametric, or tonic manner, via the cells axial stiffness. In this case the change in gain seen by the IHC does not require a phasic response at high frequencies. The $\mathrm{OHC}$ could mediate a fast acting gain control, via impedance changes, that follows the $\mathrm{OHC}$ membrane tonic voltage envelope. Given a level-dependent change in dynamic range (i.e., dynamic range compression), the tuning and sensitivity would necessarily change. Our analysis and conclusions are based upon a re-interpretation of existing mammalian outer hair cell $(\mathrm{OHC})$ studies using a generalized admittance matrix formulation of the $\mathrm{OHC}$, that relates the plasma membrane voltage and current to the soma axial force and velocity.

\section{8:15}

3aPP2. A measure of internal noise based on sample discrimination of level differences. Walt Jesteadt, Lance Nizami, and Kim S. Schairer (Boys Town Natl. Res. Hospital, 555 N. 30th St., Omaha, NE 68131)

In a sample discrimination task, subjects are presented with stimuli drawn from each of two overlapping distributions and asked to identify the distribution from which the stimuli were drawn. Lutfi [J. Acoust. Soc. Am. 86, 934-944 (1989)] has shown that performance in this task is nearly ideal when the stimuli are single sinusoids differing in level, for distributions with a mean difference of $5 \mathrm{~dB}$ and standard deviations of $5 \mathrm{~dB}$. Theoretically, as the mean difference and standard deviation of the distributions are decreased, however, performance should increasingly deviate from ideal, yielding an estimate of internal noise. To test this hypothesis, intensity discrimination was measured for seven subjects' sinusoids drawn from distributions that had mean differences ranging from $0.1-2.2 \mathrm{~dB}$, in steps of $0.3 \mathrm{~dB}$. The standard deviation of each distribution was always half the mean difference, resulting in uniform ideal performance. Estimates of internal noise obtained by fitting a function to the observed $d$-prime values agreed with estimates obtained from psychometric functions measured using the same differences in level. Analyses of decisions on individuals trials indicated that the two observation intervals were weighted equally. [Work supported by NIDCD.]

8:30

3aPP3. Effects of the finite speed of sound in-and surgical modification of - the cochlea. Carrick Talmadge (Natl. Ctr. for Physical Acoust., Univ. of Mississippi, Oxford, MS 38677, clt@olemiss.edu) and Arnold Tubis (Univ. of California, San Diego, La Jolla, CA 92093)

Most analyses of the mechanics of the cochlear to date have involved the assumption that the cochlea is a rigid chamber filled with incompressible fluids. However, in vivo measurements of the cochlear fluid pressure or basilar membrane motion necessarily must be performed by first surgically modifying the cochlea through removing part of the cochlear bone. This modification violates the assumption of a rigid chamber, and can significantly modify the mechanics of the cochlea. Because of anatomical constraints, many of these measurements are performed near the base of the cochlea. For the species that are used (guinea pig, gerbil, chinchilla, etc.), measurements near the base necessitate the use of stimulus tones with frequencies that are too high for the assumption of an incompressible fluid to be valid. In this paper, we discuss the modifications to the cochlear mechanics necessary to accommodate both the finite speed of sound and the surgical modification of the cochlea. We show that both of these modifications can easily be incorporated into numerical cochlear model codes. Semianalytic solutions based the WKB method are also discussed.

\section{8:45}

3aPP4. Dichotic sound interference products, sound localization cues, and wave interaction at a neural level. Pantelis Vassilakis (Dept. of Ethnomusicology, UCLA, 2539 Schoenberg Music Bldg., Box 951657, Los Angeles, CA 90095-1657)

The possibility of sound interference products arising from neural wave interaction is reexamined through five dichotic experiments. It is argued that interference percepts do not arise unless sound waves interact physically. Experiment 1 examines the possibility of beating for diotic sine stimuli. In this case, diplacusis would introduce beating if neural wave interaction could result in interference products. No beating was observed. Experiments $2 \mathrm{a}, 2 \mathrm{~b}$, and $2 \mathrm{c}$ examine the effect of dichotic phase changes on sine stimuli in terms of loudness, direction, and stereo-image-width. It is shown that such phase changes lead to perceptions incompatible with 
the interference principle and consistent with claims of binaural phase differences as sound-localization cues. Experiment 3 uses two-component dichotic stimuli. The results indicate that, when two sines with slightly different frequencies are presented dichotically, the constantly shifting phase between them affects a constantly shifting localization of the twocomponent complex stimulus. Depending on the frequency difference, this shift may be interpreted as a perceived rotation of the sound and/or a timbral fluctuation, often confused with loudness fluctuation. The findings do not support the claim of sound interference products arising from neural wave interaction. They are consistent with sound localization studies and indicate that the dichotic beats reported previously must have been a misidentified rotating sensation.

\section{9:00}

3aPP5. Computation and analysis of a nonlinear nonlocal cochlear model with applications to multitone interactions in hearing. Jack Xin (Dept. of Mathematics, Univ. of Texas, Austin, TX 78712), Yingyong Qi (Qualcomm, Inc., San Diego, CA 92121), and Li Deng (Microsoft Res., Redmond, WA 98052)

A nonlinear nonlocal cochlear model of the transmission line type is studied to capture the multitone interactions and tonal suppression effects. The model can serve as a module for voice signal processing. It is a one-dimensional (in space) damped dispersive nonlinear partial differential equation (PDE) based on mechanics and phenomenology of hearing. The elastic damping is a nonlinear and nonlocal functional of basilarmembrane displacement. The initial boundary value problem is solved with a semi-implicit second-order finite difference method. Numerical results are shown on two-tone suppression from both high-frequency and low-frequency sides, consistent with known behavior. Suppression effects among three tones are demonstrated by showing how the response magnitudes of the two fixed tones are reduced as the third tone is varied in frequency and amplitude. Qualitative agreement with existing cat auditory neural data is observed. The model is thus simple and efficient as a processing tool for voice signals. Mathematical analysis of global wellposedness of the model PDE and the existence of multitone solutions will also be shown via the method of a priori estimates and fixed point theory. [Work partially supported by ARO and NSF.]
9:15

3aPP6. The effect of middle ear stiffness changes on noise hearing thresholds in presence of a high level background pure tone. Hashir Aazh (P.O. Box 17445_177, Tehran, Iran, hash_aazh@hotmail.com)

In this study the interaction of two different sounds, loud low frequency pure tone and noise in threshold level was evaluated during air pressure changes in order to determine the effects of middle ear stiffness changes on noise threshold, with presence of a high level background pure tone. Forty-two normal hearing subjects after otoscopy, audiometry, and tympanometry participated in this experiment. During the presence of a background pure tone $(220 \mathrm{~Hz}, 90 \mathrm{~dB})$, the thresholds of wide band, low pass filtered, and high pass filtered noises were measured (in dB SPL) in three ear canal air pressure conditions $(0,+300,-300$ dapa $)$ through a probe set. The pump manometer system of an AZ7 impedance audiometer was used to change the air pressure in an external auditory canal. The means of noise thresholds were significantly greater in 0 dapa than +300 and -300 dapa. But there was no significant difference between noise thresholds in +300 and -300 dapa. By increasing the middle ear stiffness, the transmission of background loud tone will decrease and ear overload is omitted, then the noise hearing thresholds will be better.

\section{9:30}

3aPP7. Design of an analog cochlear implant. Joel Flores, Jorge Becerra, and Patricia L. Ramrez (Lab. de Acustica, Unidad Profesional Adolfo Lopez Mateos, ESIME Zacatenco, Av. IPN S/N, Gustavo A. Madero, Lindavista 07738, Mexico, joelflores71@ hotmail.com)

The present work describes the development of cochlear implants, an effective methodology in the treatment of severe to profound hearing loss. A group in the acoustic section from ESIME Zacatenco is investigating the development of cochlear implants in Mexico. Cochlear implants are known to have had encouraging results for subjects with postlinguistic deafness; however, one of the great drawbacks of these implants is their cost. For this reason, this work described here counts on using interdisciplinary personnel to accomplish the construction of a cochlear implant of the low-cost analog type, without losing sight of its objective: the best possible restoration of hearing.

WEDNESDAY MORNING, 4 DECEMBER 2002

CORAL GALLERY 1, 8:00 TO 11:40 A.M.

\title{
Session 3aSA
}

\section{Structural Acoustics and Vibration: Energy Flow Methods in Vibroacoustic Analysis and Control I}

\author{
Stephen A. Hambric, Chair \\ Applied Research Laboratory, Pennsylvania State University, P.O. Box 30, State College, Pennsylvania 16804
}

Chair's Introduction-8:00

Invited Papers

8:05

3aSA1. Computing structural energy density and power flow using spectral elements. José Roberto F. Arruda and Khaled M. Ahmida (Dept. of Computational Mech., Univ. Estadual de Campinas, Cidade Univ. Zeferino Vaz, Campinas, SP 13083-970, Brazil, arruda@fem.unicamp.br)

The large deterministic models obtained using numerical methods are frequently inadequate to deal with structural dynamics problems in the audio frequency range. An alternative approach that has been used for many years is the spectral approach. After transformation to the frequency domain, structural dynamics problems are described by partial differential equations that can be solved analytically in an exact way. In the case of one-dimensional waveguides, the spectral approach may be combined with a mobility approach yielding a spectral element methodology that, in many ways, resembles the finite element method. The major drawback of this approach is the difficulty in treating two- and three-dimensional waveguides. Exact solutions exist only for plates and shells with particular geometry and boundary conditions. An approximate method has been proposed for flat plates with arbitrary 
conditions, but the element assembling is still restricted to one dimension. In this paper, different formulations for flat-plate spectral elements are presented and their advantages and drawbacks are discussed. Expressions for the computation of energy densities and power flow in frame and plate spectral elements are derived and some examples are shown to illustrate the advantages of this approach in energy flow analysis applications.

8:30

3aSA2. Spectral finite elements combined with energy flow techniques: Hybrid approach for the energy flow parameters evaluation. Mohammed Ichchou and Louis Jezequel (DSS/LTDS, UMR CNRS 5513, ECL, 69130 Ecully, France)

Energy flow parameters involved in many energy flow techniques (statistical energy analysis, or its local formats) are mainly deduced from pure analytical basic theories. However, in practice, application of SEA or alternatives to complex realistic structures suffers from the choice of subsystems and needed relevant inputs. This paper proposes a procedure which reuses existing reduced finite element modeling of the structural component in view of energy flow parameters identification. Precisely, extraction and analysis of dispersion curves for typical structures, wave numbers, and energy velocities, are first concerns in this contribution. The dispersion curves extraction is based on a spectral problem and uses a reduced finite element model. Properties of eigensolutions of the posed spectral problem are first demonstrated and some remarkable aspects in term of energy flow parameters are discussed. Definitions of energy velocity, needed for modal densities expressions associated with the spectral finite element model, are presented in depth. Ultimately, extension of the proposed formulation, in order to deal with coupled complex structural components is given. This leads to the numerical evaluation of diffusion matrix (reflection and transmission parameters), often needed in order to define coupling loss factor or equivalent energy transfer quantities. Some numerical applications are finally presented.

\section{$8: 55$}

3aSA3. Analysis and minimization of power flow in a mechanical vibration isolation system using a hybrid (active/passive) approach. Peter C. Herdic, a) Brian H. Houston, Robert D. Corsaro, and John A. Judge (Naval Res. Lab, Code 7130, Washington, DC 20375)

Implementation of active control techniques in mechanical vibration isolation systems has been a challenging problem for a number of years where numerous physical control laws have been explored. An energy-based approach to the problem involving the energy transfer or power flow through the mount into the base structure is a first-principles approach to developing control laws and evaluating the system performance. A lumped-parameter model of a passive-active hybrid isolation mount has been developed and validated with experimental data. The mount device has a conventional passive compliant spring, embedded force and velocity sensors, and a piezoceramic actuation layer. This study investigates a complete set of possible layer configurations, that is, the optimal placement of sensors and actuator relative to the passive compliant isolator element. A number of different local physical control laws are examined and the level of power flow through the mount is used to evaluate the performance for the matrix of possible implementations. These results will be discussed with particular emphasis placed on the optimal control configuration and laws, and the related physics. ${ }^{a)}$ Also with SFA, Inc., Largo, MD 20774.

9:20

3aSA4. Is the modal approach appropriate for analysis of energy flow? Goran Pavic (LVA, INSA-Lyon, 20 Av. A. Einstein, 69621 Villeurbanne, France)

Modal superposition is a most commonly used approach in a numerical analysis of vibration. However, the computation requirements of a typical analysis of energy flow limit the attractiveness of the modal approach because, as a rule, a very large number of modes have to be taken into account in order to produce realistic results. The reason for this particularity is that the energy analysis involves not only vibration displacements but also higher derivatives of these which are contributed by higher modes, the higher the derivative order. More careful analysis of structure-borne vibration shows that the modal truncation is not the only inconvenience where the modal approach is used. An equally important factor limiting its use is the representation of vibration dissipation by modal damping. The paper shows comparisons of computed energy flow in plates using modal and wave approaches. The differences between the two are noticeable, in particular where the vectorial functions of energy flow field, divergence and curl, are concerned. The wave approach to vibration analysis is shown to be more physically consistent than the modal approach.

\section{9:45-10:00 Break}

10:00

3aSA5. Summary of energy flow measurements and calculations made on the INCE standard test structures. Stephen A. Hambric (ARL/Penn State Univ., P.O. Box 30, State College, PA 16804, sah@wt.arl.psu.edu)

In 1996 a series of standard test structures was conceived and manufactured by members of the Institute of Noise Control Engineering (INCE) [Cuschieri, Burroughs, and Carroll, Evaluation of Structure-Borne Noise Prediction Techniques Review, Proceedings of Noise-Con 98, April 1998, pp. 315-320]. The structures include a Lexan T-shaped beam and two ribbed panels of identical geometries but different materials: aluminum and lexan. In subsequent years, a wide variety of investigators from the U.S. and around 
the world have conducted experimental and numerical studies on the test structures, particularly on energy flow parameters, such as power input, power dissipation, and power flow. Most of the studies have been performed at low frequencies, and have shown phenomena such as the conversion of flexural wave power to longitudinal wave power at the $T$-beam joint and the nature of the structural intensity fields in the ribbed panels. Measurements and computations compare well. At high frequencies, Statistical Energy Analysis (SEA) techniques have shown that energy tends to become trapped in the drive leg of the T-beam. SEA studies on the ribbed panels show that the Lexan panel transmits less energy across the ribs than the aluminum panel does due primarily to differences in material loss factor.

$10: 25$

3aSA6. Active minimization of acoustic energy density in enclosed sound fields. Scott D. Sommerfeldt and Benjamin M. Faber (Dept. of Phys., Brigham Young Univ., Provo, UT 84602)

Minimization of acoustic energy density has been investigated for active noise control applications for enclosed sound fields. The standard approach of minimizing the squared acoustic pressure has been shown to often lead to localized control of the sound field, which may be undesirable. It has been shown that minimizing the energy density often leads to improved global attenuation of the field, since quantities dependent on both the pressure and particle velocity are minimized. This penalizes active control solutions in which the pressure is minimized and the particle velocity is increased, as often occurs when simply minimizing the squared pressure. Minimizing the acoustic energy density also requires multiple microphones to be integrated as part of the error sensor. However, in many cases the number of microphones can be reduced by strategically placing the sensor in a location where one component of the particle velocity is assumed to be negligible. Active control results will be shown for an enclosed sound field to compare the reduction obtained using energy density with that obtained for minimizing squared pressure. In addition, the attenuation obtained using a full three-dimensional energy density sensor will be compared with the control achieved using an error sensor with fewer microphones.

10:50

3aSA7. Effects of cross-sectional variation in T-beam structures. Alberto Coronado-Matutti and Rubens Sampaio (Depto. de Eng. Mecanica, PUC-Rio, Rua Marques de Sao Vicente 225, 22453-900 Rio de Janeiro, Brazil)

Structural modification of geometry has been proven to be very effective when it was necessary to improve the dynamic performance of some structures [A. J. Keane, J. Sound Vib. 185(3), 441-453 (1995)]. This technique has the advantage of achieving good performance even without the heavy viscoelastic treatments or the high-tech, high-cost active vibration control schemes. In this paper a T-beam structure [R. P Szwerc, C. B. Burroughs, S. A. Hambric, and T. E. McDevitt, J. Acoust. Soc. Am. 107, 3186-3195 (2000)] of uniform cross section is modified in order that the power transmitted to some point, due to an external excitation, is minimized. The modification of geometry is made only in one of the legs of the T-beam. The structure will be modeled combining the finite element method and the spectral element method. The dynamic response of the structures (initial and modified configurations) will be carried between $0-350 \mathrm{~Hz}$; the first ten resonances are placed in this frequency range. After defining a specific region to be optimized via genetic algorithms, the options to minimize the frequency response will be shown; in some cases it is possible to move one or more resonances, in others only a reduction on the response between the nonresonance frequencies is permitted.

\section{1:15}

3aSA8. Combining statistical energy analysis and finite element analysis in RESOUND mid frequency vibroacoustic analysis. Bryce K. Gardner, Philip J. Shorter, and Paul G. Bremner (Vibro-Acoust. Sci., 12555 High Bluff Dr., Ste. 310, San Diego, CA 92130)

At low frequencies, vibroacoustic systems exhibit a dynamic response characterized by spatially correlated motion with low modal density. These systems are typically modeled with deterministic methods. While at high frequencies, the dynamic response is characterized by weak spatial correlation and a large number of modes with high modal overlap. These systems are typically modeled with statistical methods. However many vibroacoustic systems have some regions with high modal density and some regions with low modal density. Such systems require a midfrequency solution technique. One such method has been developed based on a hybrid approach combining finite element analysis (FE) in the low modal density regions and statistical energy analysis (SEA) in the high modal density regions. This method is called RESOUND [Langley and Bremner, J. Acoust. Soc. Am. 105, 1657-1671 (1999)]. Recent developments of RESOUND have focused on predicting the appropriate dynamic interactions and mechanisms for energy flow between the FE and the SEA regions. By including these effects, RESOUND can predict the dynamic response of systems having regions with low modal densities and regions with high modal densities. This paper will provide an overview of recent developments. 


\title{
Session 3aSC
}

\section{Speech Communication: Voice, Processing and Phonetic Algorithms (Lecture/Poster Session)}

\author{
Sergio Suarez Guerra, Cochair \\ Laboratorio de Sistemas Digitales, CIC-IPN, Instituto de Cibernetica, Matematica y Fisica, ICIMAF, \\ Calle 15 \#551, Vedado Cuidad de La Habana, Cuba \\ Maria E. Hernandez-Diaz Huici, Cochair \\ CEETI, UCLV, C. Camajuani Km 5 1/2, Santa Clara 54830, Cuba \\ Diane Kewley-Port, Cochair \\ Indiana University, 501 North Morton, Bloomington, Indiana 47404
}

Chair's Introduction-8:30

Invited Papers

8:35

3aSC1. Extraction and visualization acoustic contour of the voice signal, its use as a method for the implementation and rehabilitation of voice and speech. Sergio Suarez Guerra (Laboratorio de Sistemas Digitales, CIC-IPN, Instituto de Cibernetica, Matematica y Fisica, ICIMAF, Calle 15 \#551, Vedado Cuidad de La Habana, Cuba)

The possibility to see what is said has resulted in something quite new, as a method for the implementation and rehabilitation of voice and speech. The immediate visualization of the principals acoustic parameters of the voice signal, in a graphic form, associating them with images that represent what was said, resulted in an additional alternative very stimulant in the field of Phoniatry, with applications in Special Schools. In researches done in the Special Schools during one year, results denoted a sustained advance in the learning of correct diction, from students, that in addition to the traditional method used an extraction and visualization acoustic parameters system. This system, Exparam V.2.0, has been proposed for its introduction at a national level into the Special School of the Republic of Cuba and the final statistics of its use will be ready at the end of the course 2002/2003. At the CIC/IPN, Mexico D.F., is at conclusion another system, which will support the Phoniatry clinic consulting room management using voice analysis in the same manner involving the acoustic profile representation and the inclusion of the patient's Clinic data base. This system is being tested at the Human Communication Institute. (To be presented in Spanish.)

9:05

3aSC2. Desirable characteristics in algorithms for measurements of features in pathological voices. Maria E. Hernandez-Diaz Huici (CEETI, UCLV, Santa Clara 54830, Cuba)

In this paper a critical review of some reported algorithms is made, regarding the objective measurement of features in pathologic speech. The discussion is grouped in the following topics: prosody, pith related measurements, hoarseness and vowel/consonant production. The prosody topic includes measurements of timing, stress, tempo and intonation. Pitch related measurements involve the algorithms based on pitch period, hoarseness groups measurements of noise and turbulence related to the pharyngeal functioning, while vowel/consonant production includes formant's transitions, voice onset time and nasality perturbations. Every algorithm was analyzed taking into account the following aspects: Domain of analysis, sampling frequency, sample of speech or voice used, amount of patients and kind of disease, correlation with subjective evaluations. Limitations of analyzed algorithms are shown and the desired characteristics for each topic's measures are proposed. (To be presented in Spanish.)

9:35

3aSC3. Speech intelligibility training using automatic speech recognition technology. Diane Kewley-Port (Commun. Disord. Technol., Inc. and Indiana Univ., 501 N. Morton, \#215, Bloomington, IN 47404, kewley@indiana.edu), Jonathan Dalby, and Deborah Burleson (Commun. Disord. Technol., Inc., Bloomington, IN 47404)

This paper describes two computer-based speech training systems that have been developed over the past decade of research. This research began at Indiana University and has been continued and commercialized at Communication Disorders Technology, Inc. The Indiana Speech Training Aid (ISTRA) uses a speaker-dependent speech recognizer and is designed for use by speech-language pathologists treating clients with articulation disorders of various sorts. The HearSay system employs a speaker-independent recognizer and is designed to improve the segment-level intelligibility of learners of English as a second language. Both systems employ minimal-pairs contrast training and share the underlying assumption that effective speech training requires intensive individual practice accompanied by evaluative feedback. The key technological achievements of ISTRA and HearSay are the methods employed 
for providing valid feedback on the intelligibility of the client's speech. This paper will describe these methods and report the results of new training studies that demonstrate that computer-based speech training can be an effective method for improving the English speech intelligibility of learners of English as a second language. [Work supported by Grants No. DC00893 and No. DC02213 from the NIH-National Institute on Deafness and Other Communication Disorders.]

\section{0:05}

3aSC4. String kernels for the classification of speech data. John Ch. Goddard Close, Fabiola M. Martinez Licona, Alma E. Martinez Licona (Dept. of Elec. Eng., Universidad Autónoma Metropolitana Iztapalapa, México City 09340, Mexico), and H. Leonardo Rufiner (Universidad Nacional Entre Ríos, Oro Verde, Entre Ríos 3100, Argentina)

Support Vector Machines (SVM) have been applied to a wide variety of classification problems with excellent results. This has to do with their provable generalization ability derived from Statistical Learning Theory. Recently specialized kernels, such as the Fisher kernel and string kernels, have been introduced in an attempt to apply the same SVM framework to sequential data. Notable results have been obtained on classification tasks related to biosequences and text documents showing that the specialized kernels may provide a viable and interesting alternative to other classifiers, such as those using Hidden Markov Models. String kernels are particularly attractive because of their conceptual simplicity and they also furnish insight into the task of sequential data classification. In the present paper string kernels are applied to a new application area, that of automatic speech recognition. In particular, different string kernels are tested on the task of phoneme recognition and the results obtained are compared with those from several other common classifiers. This comparison reveals the potential of string kernels as a simple and feasible alternative to other established methods for certain speech recognition tasks. [Work supported by CONACYT under Project 31929-A.]

\section{Contributed Papers}

All posters will be on display from 10:35 a.m. to 12:00 noon. To allow contributors an opportunity to see other posters, contributors of odd-numbered papers will be at their posters from 10:35 a.m. to 11:15 a.m. and contributors of even-numbered papers will be at their posters from 11:15 a.m. to 12:00 noon.

3aSC5. Technical considerations in the design of a wearable voice dosimeter. Peter S. Popolo, Karen Rogge-Miller, Jan G. Svec, and Ingo R. Titze (Wilbur James Gould Voice Ctr., The Denver Ctr. for the Performing Arts, 1245 Champa St., Denver, CO 80206, ppopolo@dcpa.org)

This paper deals with the technical issues involved in the design of a voice dosimeter, a wearable device to be used by teachers to measure vocal dose on the job, at home and elsewhere during the total waking hours of each day. The concept of vocal dose is introduced and definitions of various dose measures are presented. Practical issues of the design are discussed, including software development, selection of an external transducer, and devising a comfortable, reliable and repeatable means of attaching the transducer to the subject. The dosimeter software was designed to calculate SPL (sound pressure level), $F 0$ (fundamental frequency) and voicing time. In addition, an interactive portion was developed that prompts the user to verify the validity of the stored data every two hours, and to perform a rating of vocal effort and quality of soft phonation. The SAL (surface acceleration level) to SPL relation for the prototype dosimeter was determined. The prototype has been field-tested, and SPL, F0, and voicing time data have been analyzed. A sensitivity analysis shows that small errors in SPL have a large effect on the accuracy of dose calculations, while $F 0$ errors have less of an effect.

3aSC6. Neural-network-based voice-tracking algorithm. Mary Baker, Charise Stevens, Brennen Chaparro (Dept. of Elec. Eng., Texas Tech Univ., M.S. 3102, Lubbock, TX 79409, mcb@coe.ttu.edu), and Dwayne Paschall (Texas Tech Univ., Lubbock, TX 79409)

A voice-tracking algorithm was developed and tested for the purposes of electronically separating the voice signals of simultaneous talkers. Many individuals suffer from hearing disorders that often inhibit their ability to focus on a single speaker in a multiple speaker environment (the cocktail party effect). Digital hearing aid technology makes it possible to implement complex algorithms for speech processing in both the time and frequency domains. In this work, an average magnitude difference function (AMDF) was performed on mixed voice signals in order to determine the fundamental frequencies present in the signals. A time prediction neu- ral network was trained to recognize normal human voice inflection patterns, including rising, falling, rising-falling, and falling-rising patterns. The neural network was designed to track the fundamental frequency of a single talker based on the training procedure. The output of the neural network can be used to design an active filter for speaker segregation. Tests were done using audio mixing of two to three speakers uttering short phrases. The AMDF function accurately identified the fundamental frequencies present in the signal. The neural network was tested using a single speaker uttering a short sentence. The network accurately tracked the fundamental frequency of the speaker.

3aSC7. Diagnostic system for speech articulation and speech understanding. Andrzej Czyzewski, Bozena Kostek, and Henryk Skarzynski (Inst. of Physiol. and Pathol. of Hearing, Pstrowskiego 1, Warsaw, Poland)

With the increase in access to multimedia computers, speech training can be made available to patients with no continuous assistance required from speech therapists. Another function the system can easily perform is screening testing of speech fluency providing directed information to patients who have various speech disorders and problems with understanding speech. The idea underlying the proposed system is a programmed speech therapy training algorithm consisting of diagnostic tools and rehabilitation devices connected with it. The first function the system described in the paper has to perform is data acquisition where information about the patient's medical history is collected. This is done through electronic questionnaires. The next function is analysis of the speech signal articulated by the patient when prompted by the computer followed by some multimedia tests carried out in order to assess the subject's ability to understand speech. Next, the results of the electronic questionnaire, the patient's voice, and the patients' reactions are automatically analyzed, based on that the system automatically diagnoses possible speech disorders and how strong they are. A large number of schoolchildren were tested with this method. In the paper foundations of applied speech testing method and obtained results will be demonstrated. 
3aSC8. Choice of speech features for single-channel vibrotactile stimulators. Alessio Trindade Barros, Raimundo Carlos S. Freire, and Gurdip Singh Deep (Dept. of Elec. Eng., Federal Univ. of Paraiba, Av Aprigio Veloso, 882, Campina Grande, PB, Brazil)

The choice of speech features for coding of a single-channel vibrotactile stimulation signal was analyzed to obtain a better discrimination between phonemes that compose each viseme of the Portuguese language. Two different speech features were selected for comparison, based on previous investigations [I. R. Summers and D. Gratton, IEEE Trans. Rehab. Eng. 3, 117-121 (1993)]: the amplitude envelope-AE and the zerocrossing frequency-ZRX of the speech signal. A codification scheme (PRP) was proposed, with output based on the switching between $\mathrm{AE}$ and ZRX, depending on the value of ZRX. The selected voice items were consonants of each viseme, in the context vCv: ata/ada/ana, apa/aba/ama, aka/aga/alha, asa/aza, afa/ava, acha/aja, and ala/ara, for different combinations of vowels. Histograms for each feature were done for each consonant of a viseme, and were compared in the context of each viseme. It was observed that the output of PRP was the same as AE for most of the consonants, but different for fricatives. PRP allowed better discrimination than $\mathrm{AE}$ and ZRX alone. The difficulty of single-channel stimulator with discrimination of fricatives is known in the literature, and the proposed scheme showed better results for this work. [Work supported by CAPES.]

3aSC9. Speech coding for fast speech commands recognition. Abel Herrera and Arturo Gardida (Universidad Nacional Autonoma de Mexico (UNAM), Electrica, 2o piso, Edificio Posgrado, Fac. Ingenieria, Circuito Exterior, CU, DF, 04510, Mexico)

Isolated speech recognition could be more appropriate than continuous speech recognition systems for some word command applications. In this paper, some speech coders are explored that can be useful for fast word command recognition using low memory storage. First, decimated critical bands vectors by frame are used as coding features. The accuracy is around $94 \%$ for a set of ten commands; they are used at first step. After that, using a reduced DTW classification scheme, the accuracy for a small subset of possible candidates is above $99 \%$. Another coding scheme is using the Karhunen-Loeve Transform (KLT) combined with multisection vector quantization. The KLT is not a fast transform; however, used at the subword level it gives a fast recognizer with accuracy around 99\%. The classifiers in both cases are fast, around a second, and require low memory storage.

3aSC10. Adapted speech recognizer to improve English pronunciation of Hispanics. Maria A. Garcia and Rene O. Arechiga (UAM-A, Depto. Electronica, Av. San Pablo \#180 Col Reynosa Tamaulipas, Azc. Cp. 02200 Mexico D.F., arechiga@correo.azc.uam.mx)

This work presents advances on an adapted speech recognition system, based on hidden-Markov models (HMMs), to help Hispanics in their pronunciation of English. The main results so far, with English digits, show significant recognition improvements of the adapted recognizer over the not adapted one, both for one speaker (100\% vs $94 \%)$ and for groups of them $(96 \%$ vs $74 \%)$. The adaptation uses maximum likelihood linear regression (MLLR). Two databases were used in the experiments. The TIMIT database to train the recognizer models with English native speakers, and the Latin-American Spanish database to adapt and test the adapted and not adapted recognizers. The project, of which this work is a part, is an English pronunciation tutor, whose main parts are this adapted speech recognizer, a pronunciation evaluator (phonetic and prosodic) and a dialog manager. English speech recognition systems are trained with native speakers, and most of the recognition errors are attributed to the interference of foreign accents. This is why it is necessary to adapt the models to take into account the characteristic features of a given population; in this case Hispanics. (To be presented in Spanish.)
3aSC11. Phone inventory optimization for multilingual automatic speech recognition. Lynette Melnar and James Talley (Motorola Labs, 7700 W. Parmer Ln., MD: PL26, Austin, TX 78729)

This paper describes a phone inventory optimization procedure for application in multilingual automatic speech recognition (ASR). The optimization procedure is based on three knowledge sources that act collectively to guide phonological reduction and selection processes: (1) abstract (language-independent) phonological universals and tendencies that are used in the construction of a hierarchical structure that specifies phone class reduction paths; (2) language-dependent knowledge that includes information of the targeted languages' phone inventories and individual phone frequencies in language data resources; (3) acoustic data that provides phone discriminability and similarity metrics. Using the optimization procedure, the phone inventories of six languages, American English, Mandarin Chinese, Egyptian Colloquial Arabic, Japanese, German, and Spanish, were merged to create an inventory consisting of 64 distinct cross-phonological units. This reduced phone set was used in all training and testing procedures and resources for the recognition of the six targeted languages. Preliminary recognition results are very encouraging: while purely data-driven approaches to multilingual ASR fail to reach wordrecognition rates comparable to monolingual applications, the use of the optimized phone inventory in our multilingual ASR program yields recognition rates approximating that of monolingual ASR.

3aSC12. Energy redistribution speech intelligibility enhancement, vocalic and transitional cues. John G. Harris and Mark D. Skowronski (Computational Neuro-Eng. Lab., Univ. of Florida, Gainesville, FL 32611, harris@cnel.ufl.edu)

Two novel speech enhancement algorithms are presented that automatically increase intelligibility in noisy environments while maintaining the signal power and naturalness of the original speech. These energy redistribution (ER) algorithms move signal energy to targeted regions of relatively high information content that are crucial for intelligibility. The boosted regions are originally of low energy and therefore usually the first segments lost with the addition of environmental noise. The ER voiced/ unvoiced (ERVU) method transfers energy from voiced speech to regions of unvoiced speech, while the ER spectral transition (ERST) method moves energy from spectrally stationary regions to spectrally transitional regions. Hand-held cell phones and public address systems are expected to be the dominant applications for these techniques. Standard noise reduction methods such as spectral subtraction are assumed to have already been applied to the voice signal before broadcast. Using human listening tests, it was found that both algorithms boost the intelligibility of speech in noisy environments by nearly $7 \%$ over the original unprocessed signals, without degrading naturalness or increasing signal power. Furthermore, both algorithms allow for controlling the trade-off between boost gain and speech naturalness.

3aSC13. The effect of speech compression on second language speech perception learning. Takahiro Adachi (ATR Human Information Sci. Labs., Kyoto 619-0288, Japan and The Grad. Univ. for Advanced Studies, tadachi@atr.co.jp) and Tsuneo Yamada (Natl. Inst. of Multimedia Education, Chiba 261-0014, Japan)

In recent years, second language education is sometimes conducted over the Internet. However, the speech used for training is often compressed in order to decrease the number of packets transmitted, without an evaluation of whether the compression causes a decrease in intelligibility. The current study assessed the intelligibility of compressed speech in order to determine whether it is appropriate for use in Internet-based training. English words minimally contrasting in $/ \mathrm{r} /$ and $/ 1 /, / b /$ and $/ \mathrm{v} /$, or $/ \mathrm{s} /$ and /th/ were recorded from native speakers of English and compressed using several different methods (MP3, WMA, QCELP, ADPCM, etc.). The intelligibility of these speech files was then evaluated by a group of native English speakers and a group of native Japanese speakers, using a minimal-pair identification task. Results showed that: (1) intelligibility 
scores for Japanese speakers were lower than those for the English speakers, as was expected; and (2) intelligibility of $/ \mathrm{r} /-/ 1 /$ stimuli was unaltered by the compression, whereas intelligibility of /s/-/th/ stimuli dropped remarkably in several formats, and that of $/ b /-/ v /$ stimuli dropped only in QCELP-compressed words. These findings suggest that some compression formats are not appropriate in speech perception training. Implications for Internet educational materials will be discussed.

3aSC14. Invariance of lognormal distribution parameters in speech segment durations. Kristin Rosen and Raymond Kent (Waisman Ctr., Univ. of Wisconsin, 1500 Highland Ave., Madison, WI 53705)

This study re-examines speech segment duration using lognormal distributions and presents a basis for the unification of many previous measurements in connected speech. Distributions of speech segment length in previous studies (Pols, Wang, and ten Bosch, 1996; Crystal and House, 1982, 1988; Duez-Goldman-Eislinger, 1968) were replotted onto lognormal cumulative plots. Speech segments were consistently symmetric on the logarithmic scale and well characterized by the lognormal distribution, with the exception of fricatives. Analysis with respect to the logarithmic scale reveals surprising consistencies within categories. Specifically, geometric standard deviation appears to be essentially preserved across phonemic categories. The nature of the distributions themselves suggests possible clues to the understanding or modeling of the underlying timing mechanisms, and provides a foundation for meaningful comparisons in connected speech. Possible advantages of logarithmic-based measures (i.e., geometric mean, geometric standard deviation) over linear measures (e.g., coefficient of variability) are presented.

3aSC15. Quantifying nonverbal communicative behavior in face-toface human dialogues. Mustapha Skhiri (Dept. of Computer and Information Sci. (IDA), Linköping Univ., SE-581 83 Linköping, Sweden, and Grad. School of Lang. Technol. (GSLT), Sweden, mussk@ida.liu.se and Loredana Cerrato (Royal Inst. of Technol., Stockholm, Sweden)

The referred study is based on the assumption that understanding how humans use nonverbal behavior in dialogues can be very useful in the design of more natural-looking animated talking heads. The goal of the study is twofold: (1) to explore how people use specific facial expressions and head movements to serve important dialogue functions, and (2) to show evidence that it is possible to measure and quantify the entity of these movements with the Qualisys MacReflex motion tracking system. Naturally elicited dialogues between humans have been analyzed with focus on the attention on those nonverbal behaviors that serve the very relevant functions of regulating the conversational flux (i.e., turn taking) and producing information about the state of communication (i.e., feedback). The results show that eyebrow raising, head nods, and head shakes are typical signals involved during the exchange of speaking turns, as well as in the production and elicitation of feedback. These movements can be easily measured and quantified, and this measure can be implemented in animated talking heads.

\title{
Session 3aSP
}

\section{Signal Processing in Acoustics and Underwater Acoustics: Acoustic Automatic Target Recognition I}

\author{
Ning Xiang, Cochair \\ National Center for Physical Acoustics, University of Mississippi, Coliseum Drive, University, Mississippi 38677 \\ Armando Andrade, Cochair \\ Calle H. No. 11, Col. Jardines de Santa Clara, Ecatepec Edo. De. CP 55450, Mexico
}

Chair's Introduction-8:30

Invited Papers

8:35

3aSP1. Automatic target recognition in acoustics: An overview. John R. Sacha (Appl. Res. Lab., The Penn State Univ., P.O. Box 30, State College, PA 16804-0030, jrs9@psu.edu)

\begin{abstract}
Automatic target recognition (ATR) constitutes one of the major uses for acoustical signal processing. ATR is employed in manned systems for operator workload reduction and performance improvement, as well as in autonomous applications. An overview of some of the major components involved in the architecture of such systems is provided. Feature extraction is the most critical step of ATR and is necessarily application specific. Generic feature selection and ranking methods are presented, including heuristic search and information-theoretic measures. Basic pattern recognition definitions and techniques are reviewed. Commonly used classification paradigms include classical statistical formulations, both parametric and nonparametric, and neural nets; support vector machines and nonmetric methods such as decision forests are some alternative techniques that have received recent attention. A few practical issues often encountered when constructing recognition systems, including training data requirements, ground truth labeling, and performance evaluation methodologies and metrics, are also addressed.
\end{abstract}


3aSP2. Advances in algorithm fusion for automated sea mine detection and classification. Gerald J. Dobeck and J. Tory Cobb (Naval Surface Warfare Ctr., Coastal Systems Station, Dahlgren Div., Panama City, FL 32407-7001, DobeckGJ@ncsc.navy.mil)

Along with other sensors, the Navy uses high-resolution sonar to detect and classify sea mines in mine-hunting operations. Scientists and engineers have devoted substantial effort to the development of automated detection and classification (D/C) algorithms for these high-resolution systems. Several factors spurred these efforts, including: (1) aids for operators to reduce work overload; (2) more optimal use of all available data; and (3) the introduction of unmanned minehunting systems. The environments where sea mines are typically laid (harbor areas, shipping lanes, and the littorals) give rise to many false alarms caused by natural, biologic, and manmade clutter. The objective of the automated $\mathrm{D} / \mathrm{C}$ algorithms is to eliminate most of these false alarms while maintaining a very high probability of mine detection and classification $(\mathrm{PdPc})$. In recent years, the benefits of fusing the outputs of multiple $\mathrm{D} / \mathrm{C}$ algorithms (Algorithm Fusion) have been studied. To date, the results have been remarkable, including reliable robustness to new environments. In this paper a brief history of existing Algorithm Fusion technology and some techniques recently used to improve performance are presented. An exploration of new developments is presented in conclusion.

9:35

3aSP3. A pulse length tolerant neural network-based detector for sector-scan sonar. Stuart W. Perry (Maritime Operations Div. Defence Sci. and Technol. Organisation, P.O. Box 44, Pyrmont, NSW 2009, Australia, Stuart.Perry@dsto.defence.gov.au) and Ling Guan (Ryerson Polytechnic Univ., Toronto, ON M5B 2K3, Canada)

In this paper we present a neural network-based system to detect small manmade objects in sequences of sector-scan sonar images created using signals of various pulse lengths. The sonar system considered has three modes of operation to create images over ranges of up to $800 \mathrm{~m}$ using acoustic pulses of different durations for each mode. After initial cleaning and segmentation to extract objects, features are computed from each object. These features consist of basic object size and contrast statistics, shape moments, moment invariants, and features derived from the second-order histogram of each object. Optimal sets of 15 features from the total set of 31 are chosen using sequential feature selection techniques. Using these features a neural network is trained to detect manmade objects in any of the three sonar modes. The proposed detector is shown to perform very well when compared with detectors trained specifically for each sonar mode and a number of statistical detectors. The proposed detector achieves a $92.4 \%$ detection probability at a mean false alarm rate of 10 per frame averaged over all sonar mode settings. Finally, research into Recurrent Neural Network detectors is described and shown to further improve performance.

10:05

3aSP4. Model-based acoustic characterization and classification of irregular-shaped targets: Application to fisheries and zooplankton acoustics. Dezhang Chu, Timothy K. Stanton, and Peter H. Wiebe (Woods Hole Oceanogr. Inst., Woods Hole, MA 02543)

Acoustic scattering by fish and zooplankton is a complicated function of the geometrical and physical properties of the targets, as well as the environmental and sonar system parameters. The shape and anatomy of zooplankton vary significantly from taxa to taxa and their dominant scattering mechanisms can be completely different. As a result, the acoustic classification of such targets is extremely difficult and often nonunique. To reduce the ambiguity and nonuniqueness, a number of model-based methods are presented. These methods use the temporal, spatial, spectral, and statistical signatures of acoustical scattering signals and can be applied to a variety of acoustic systems, including narrow-band, broadband, and multifrequency systems. The methods also depend strongly on whether or not the targets are resolved. Individual targets with different shapes and material properties have their unique characteristics and can be classified acoustically in terms of their size, orientation, scattering mechanisms, as well as their material properties. Results of applying these methods to the laboratory and field data will be presented and analyzed. [Work supported by ONR, NSF, and the Comer Science and Education Foundation.]

10:35-10:45 Break

\section{Contributed Papers}

\section{0:45}

3aSP5. A neural network approach for data inversion, application to the characterization of marine sediments, and sonar targets. Manell E. Zakharia (IRENAV, Res. Inst. of the French Naval Acad., BP 600 F29240, Brest Cedex, France)

The paper presents an innovative method for inversion based on the use of neural networks. The inversion procedure will be split in three major steps: the understanding and enhancing of the physical phenomena involved, the learning of the physics from extracted parameters, and the inversion itself. This separation allows a very convenient distribution of computation load: the first two steps (which are the most demanding in terms of computation time) can be done in a prior phase, while the third one (which becomes very simple) can be implemented on-line (real time). Another major advantage of the proposed method (with respect to conventional approaches such as conjugate gradient) is that it requires only poor a priori information thanks to a judicious choice of the characteristic physical phenomena and the selection of relevant signal processing tools for parameter extraction. Two examples of the proposed procedure are described: the inversion of geoacoustic parameters of seabed and the characterization of sonar targets. The inversion is applied to both simulated and experimental data sets. In both cases, the training will be achieved on simulated data and then applied to the experimental. 
3aSP6. Real time identification of several targets by iterative time reversal. Gabriel Montaldo, Mickael Tanter, and Mathias Fink (Laboratoire Ondes et Acoustique, Universit Paris VII, ESPCI, CNRS UMR 7587, Paris, France)

Finding punctual targets inside an aberrating medium is a problem of interest in nondestructive testing and medical research. Previous works have shown that the iteration of the time reversal method allows us to identify the strongest reflector of the medium in real time but the identification of several targets is not possible with this technique. Using the idea that each strong scatterer is associated to an eigenvalue of the time reversal operator, a method has been developed (the DORT method) to identify punctual diffusers in a medium. This technique requires the experimental acquisition of the whole time reversal operator and its numerical decomposition implies many calculations. We propose here a way to identify in real time all the punctual targets by modifying the time reversal iterative method. In a first step, we identify the strongest reflector by time reversal processing. In a second step, we subtract its contribution in order to find the second reflector contribution and so on. This method allows us to build an "eigen pulse" able to focus a pulsed signal on each target. We demonstrate experimentally its real time feasbility by identifying several targets inside a strongly aberrating medium.
3aSP7. Ultrasonic sensor system to detect solids in a milk pasteurization process. Carlos Barroeta Z., Fernando L. Sanchez M., G. Moreno Fernando R., and Laura Montes P. (Control Lab., ESIME, IPN, Mexico, cbarroet@att.net.mx)

In the food industry, many products require a specific process. In the milk industry, the raw milk passes through several process stages before reaching the end user in a very qualitative and healthy way. One of the problems of the milk is that it can contain solids in suspension, result of contamination of the milk, or inherent to the pasteurization process itself. In order to control these solids, a solid detection system is being developed, which will detect the solids by the reflection and refraction of ultrasonic waves. The sensor must be set in the upper part of the milk containers, and with a grid array to allow the control system to prevent these solids from entering into the pipes of the processing plant. The sensing system may activate an acoustic alarm to indicate that a solid has been detected, and a visual one to indicate the affected part of the process. (To be presented in Spanish.)

\title{
Session 3aUW
}

\section{Underwater Acoustics and Acoustical Oceanography: Littoral Environmental Variability and Its Acoustic Effects I}

\author{
Finn B. Jensen, Cochair \\ SACLANT Undersea Research Center, Viale San Bartolomeo 400, 19138 La Spezia, Italy \\ James F. Lynch, Cochair \\ Woods Hole Oceanographic Institution, 203 Bigelow Building, Woods Hole, Massachusetts 02543 \\ Chair's Introduction-7:50 \\ Invited Papers \\ $7: 55$
}

3aUW1. Results from a series of acoustic variability experiments. Martin Siderius (SAIC, 1299 Prospect St., La Jolla, CA 92037, sideriust@saic.com), Peter Nielsen (SACLANT Undersea Res. Ctr., 19138 La Spezia, Italy), and Jurgen Sellschopp (FWG, 24148 Kiel, Germany)

A series of experiments were conducted in recent years by the SACLANT Undersea Research Centre to measure the effect of the environment on acoustic propagation. These broadband, shallow water experiments used a moored vertical array with both towed and fixed sound sources. During the fixed experiments the sound source was mounted on a steel frame tower that was resting on the seabed. This design was used to isolate the acoustic fluctuations caused by the environment from those due to source motion. During transmissions extensive measurements of the environment were made including range-depth profiling of the ocean sound speed along the propagation track using a vertically towed CTD (conductivity, temperature, and depth) chain. The seabed types at the sites varied from soft clay (sound speed less than in the water) to a highly reflective sand-gravel (sound speed about $1750 \mathrm{~m} / \mathrm{s}$ ). The temporal fluctuations and spatial structure of the received acoustic signals varied greatly between the sites. This had a big impact on propagation modeling for transmission loss; matched-field processing; and model-based, geo-acoustic inversion. In this paper an overview of these acoustic variability experiments will be described along with results showing the effect of the environment on model-based processing.

\section{$8: 15$}

3aUW2. Acoustic propagation in the coastal environment of the Florida Straits—recent experimental results. Harry Deferrari, Neil Williams, and Hien Nguyen (RSMAS, Univ. of Miami, Miami, FL 33138)

An autonomous source was moored at ranges of 10 and then $20 \mathrm{~km}$ from a vertical receiver array with 32 elements in a depth of $145 \mathrm{~m}$ of water. $M$ sequences were transmitted for 28 days at six center frequencies from 100 to 3200 in one octave increments. Arrivals and paths are identified with models and then fluctuation statistics, coherence, and predictability are examined in a parameter space of frequency, range, and receiver depth. A group of refracted-bottom-reflected (RBR) modes/rays has nearly equal group 
velocities and tends to focus in time and depth forming intense arrivals especially at the depth of the transmitted. A second group of surface reflected bottom reflected (SRBR) modes produce arrivals that fan out in time. Coastal areas inside western boundary currents have exceptionally variable sound speed fields owing to dynamical effects such as meanders, shelf waves, eddies, coastal upwelling and energetic internal waves and tides. Sound speed fluctuations are observed to be an order greater than the deep ocean. Very large changes in mean sound speed profiles and extreme gradients occur at subinertial periods. Also, potential energy of the internal wave field varies with the same longer periods as do statistical properties of observed acoustic signals.

\title{
8:35
}

3aUW3. Modeling the interaction of acoustic and internal wave fields in shallow-water environments. Steven Finette (Acoust. Div., Naval Res. Lab., Washington, DC 20375)

Over the past decade, there has been considerable interest in the effect of solitary wave packets on the acoustic field. This talk will present an overview of modeling efforts to describe the interaction of these internal gravity waves with acoustic field propagation in shallow-water waveguides. The emphasis will be on the interaction of solitary wave packets with low-frequency acoustic fields propagating in a continental shelf/slope environment. Topics to be discussed will include both adiabatic and mode-coupling effects, differences between 2-D and 3-D propagation, as well as the influence of wave packets on horizontal array beamforming. Most research to date involves solitary wave propagation over flat bathymetry, but does not address the generation of internal tides over variable bathymetry and their subsequent evolution into wave packets. In this regard, recent nonlinear fluid dynamic simulations of both the generation and propagation of internal tides and wave packets are illustrated and their influence on acoustic propagation briefly addressed. [Work supported by ONR.]

\section{8:55}

3aUW4. Rapid fluctuations of the channel impulse response at midfrequencies in shallow water. W. S. Hodgkiss, W. A. Kuperman, and D. E. Ensberg (Marine Physical Lab., Scripps Inst. of Oceanogr., La Jolla, CA 92093-0701, wsh@mpl.ucsd.edu)

\begin{abstract}
A fixed source, fixed receiving array experiment was carried out to measure the stability of forward transmissions in shallow water ( $\sim 100 \mathrm{~m}$ deep) over a $6 \mathrm{~km}$ forward propagation path off San Diego, CA. The source was moored $6 \mathrm{~m}$ above the seafloor and the 12 aperture, 64-element vertical receiving array was deployed with the lowest element $4 \mathrm{~m}$ off the bottom. The source transmissions of interest here are the $2 \mathrm{kHz}$ bandwidth, $1 \mathrm{~s}$ duration FM chirps which were transmitted continuously for $5 \mathrm{~min}$ at a time and have been matched filtered to yield the channel impulse response. In addition to CTDs taken in the region between the source and receiving array, a thermistor string at the receiving array site provided measurements of water column temperature fluctuations. The timeevolving structure of the channel impulse response clearly shows significant, environmentally induced fluctuations which also are evident in an arrival angle vs travel time spatial decomposition at the array. [Work supported by ONR.]
\end{abstract}

\section{Contributed Papers}

\section{9:15}

3aUW5. High frequency sonar variability in littoral environments: Irregular particles and bubbles. Simon D. Richards (QinetiQ, Winfrith Technol. Ctr., Dorchester, Dorset DT2 8XJ, UK), Timothy G. Leighton, and Paul R. White (Univ. of Southampton, Highfield, Southampton SO17 1BJ, UK)

Littoral environments may be characterized by high concentrations of suspended particles. Such suspensions contribute to attenuation through visco-inertial absorption and scattering and may therefore be partially responsible for the observed variability in high frequency sonar performance in littoral environments. Microbubbles which are prevalent in littoral waters also contribute to volume attenuation through radiation, viscous and thermal damping and cause dispersion. The attenuation due to a polydisperse suspension of particles with depth-dependent concentration has been included in a sonar model. The effects of a depth-dependent, polydisperse population of microbubbles on attenuation, sound speed and volume reverberation are also included. Marine suspensions are characterized by nonspherical particles, often plate-like clay particles. Measurements of absorption in dilute suspensions of nonspherical particles have shown disagreement with predictions of spherical particle models. These measurements have been reanalyzed using three techniques for particle sizing: laser diffraction, gravitational sedimentation, and centrifugal sedimentation, highlighting the difficulty of characterizing polydisperse suspensions of irregular particles. The measurements have been compared with predictions of a model for suspensions of oblate spheroids. Excellent agreement is obtained between this model and the measurements for kaolin particles, without requiring any a priori knowledge of the measurements.
3aUW6. Sediment tomography in the East China Sea: Compressional wave speed and attenuation inversions from Airy phase dispersion measurements and time series correlation. Colin Lazauski, James Miller, Gopu Potty, Chuen-Song Chen (Dept. of Ocean Eng., Univ. of Rhode Island, Narragansett, RI 02882), and Peter Dahl (Univ. of Washington, Seattle, WA 98105)

This paper discusses ongoing data analysis results from the acoustic bottom interaction experiment conducted in May-June 2001 in the East China Sea as part of the Asian Seas International Acoustics Experiment (ASIAEX-2001). Using time-frequency scalograms of broadband signals, the modal arrivals and group speed minimums (Airy Phase) of several modes are clearly observed. The structure of the Airy Phase signal is used to match the dispersion curves which forms the basis of this inversion technique. Utilizing the Airy Phase group speed minimums and corresponding pressure amplitudes of each observable mode, the sediment compressional wave speed and attenuation as a function of depth are derived. The group speed minimum for each mode provides additional information on the compressional wave speed in the modal sediment depth penetration interval. To refine the sediment parameters, synthetic and measured time series are correlated for goodness of fit and used in the inversion process. The synthetic time series is generated from the scalogram values corresponding to the times and frequencies of the calculated dispersion curves. Inverted speeds and estimated modal penetration depths are then used to develop the sediment profile. The estimated resolution is dependent on the number and frequency span of the observable modes. Estimated sediment properties from several areas are presented with verification from coring results. [Work supported by ONR.] 
3aUW7. Inversion of geoacoustic parameters in the South China Sea. Gopu R. Potty, James H. Miller, Colin J. Lazauski (Dept. of Ocean Eng., Univ. of Rhode Island, Narragansett, RI 02882), James F. Lynch, and Arthur Newhall (Woods Hole Oceanogr. Inst., Woods Hole, MA 02543)

During the ASIAEX Experiment in the South China Sea, light bulb sources were deployed in the vicinity of the receive array in order to provide array element localization. We present inversions for bottom geoacoustic properties using these broadband light bulb data. The light bulbs imploded about $40 \mathrm{~s}$ after launch and were deployed approximately $1 \mathrm{~km}$ from the receive array. The receive array was at a depth of $125 \mathrm{~m}$ of water. The ground truth measurements include bathymetry data from ship board measurements, chirp sonar data, and sediment cores. SeaSoar surveys and CTD measurements provide sound-speed data in the water column. The inversion is carried out using a coherent broadband matched field processing technique [Chapman et al., Oceans 97, MTS/IEEE Conference Proceedings, Vol. 2, pp. 763-768]. The thickness of the top layer of the sediment and the compressional wave speeds in the top and bottom layers are estimated in the vicinity of the receive array and compared with ground truth measurements. [Work supported by ONR.]

\section{0:00-10:15 Break}

\section{0:15}

3aUW8. Horizontal wavenumbers from a fluctuating waveguide. Robert Field, Joal Newcomb, James Showalter, and Jacob George (Naval Res. Lab., Stennis Space Center, MS 39529)

From September 26 through November 2, 2000 the U.S. and Japan conducted a joint experiment in the New Jersey Bight off the U.S. East Coast. The experiment was conducted in three legs, the first of which investigated low frequency acoustic fluctuations in the presence of a shelf/ slope front and internal waves. Legs II and III investigated acoustic scattering at $5.5 \mathrm{kHz}$ and methods of acoustic inversion for sea floor properties, respectively. In this paper we will focus on the experimental results of leg I and discuss implications for doing acoustic inversions in a strongly fluctuating medium. Theoretical results based on the Pekeris waveguide show that a large-scale, slowly fluctuating sound speed can cause rapid fluctuations in the acoustic field in the form of acoustic harmonics. Pekeris waveguide calculations are compared with a subset of the acoustic data. Theoretical results and parabolic equation simulations are used to show the impact of the water column sound speed on the horizontal wavenumbers and hence on acoustic inversion methods that utilize them. [Work supported by ONR, Program Element PE62435N.]

\section{0:30}

3aUW9. A broadband ocean sediment acoustics model for signal processing applications. Nicholas P. Chotiros and Marcia J. Isakson (Appl. Res. Labs., Univ. of Texas, P.O. Box 8029, Austin, TX 78713-8029, misakson@arlut.utexas.edu)

It has been shown that fluid and viscoelastic solid approximations cannot accommodate the observed sound speed dispersion and enhanced reflection loss over sandy shallow water sediments. A plausible poroelastic model has been developed for the high frequency band $(>50 \mathrm{kHz})$ using measurements from several sources. This model, with constant coefficients, is unable to track the observed sound speed dispersion at lower frequencies. It is hypothesized that one parameter, the frame bulk modulus, varies with frequency in a relaxation process associated with squirt flow at the grain-grain contact. This hypothesis has the potential to be a critical component in broadband acoustic models of granular ocean sediments. It will link measurements at high frequencies to propagation modeling at low frequencies, provide accurate, physics based, models of propagation loss, and a means to invert for bottom properties over a broad range of frequencies. [Work supported by ONR, Undersea Signal Processing.]
3aUW10. Coupled electrokinetic-Biot theory and measurement techniques in sediment acoustics. Gareth Block and Nicholas Chotiros (Appl. Res. Labs., Univ. of Texas, P.O. Box 8029, Austin, TX 78713-8029, gblock@arlut.utexas.edu)

Biot theory will no doubt play an important role in modeling wave propagation in ocean sediments. One prediction of Biot theory is the existence of the Biot slow wave, described by out-of-phase motion of the pore fluid and porous grain structure. Unfortunately, slow wave motion has been extremely difficult to verify using acoustical transducers. Electrokinetic (EK) transduction techniques shed new light on this effort. EK parameters, such as the surface chemistry of the grains and pore fluid salinity, are coupled to Biot theory at the microscopic level, and therefore provide another method of determining the microstructural properties of sediments. One application of this is the electrokinetic transmission of acoustic pressure waves. This occurs when an applied voltage drives ionic (and thus fluidic) currents in electrolyte-saturated porous media. Experimental and theoretical results for electrokinetic motion in sediments will be reported. [Work supported by ONR, Ocean Acoustics.]

\section{1:00}

3aUW11. Environmental influences on the frequency dependence of effective bottom attenuation. James D. Nickila (Adv. Sonar Technol. Div. Naval Undersea Warfare Ctr., Newport, RI 02841) and Kevin B. Smith (Naval Postgrad. School, Monterey, CA 93943)

Over the past several years, concern has grown over the appropriateness of bottom attenuation models that assume a linear frequency dependence. Empirical analyses of experimental data have suggested power-law dependence with frequency exponents as high as 1.7 and above, but with large variability between geographic regions [Zhou et al., J. Acoust. Soc. Am. 82, 287-292 (1987)]. The fundamental cause of this dependence is unknown. In this analysis, the influence of the propagation and interaction with environmental variability is investigated. Specifically, a propagation model that assumes linear frequency dependence is employed which incorporates such environmental variability as range-dependent water column sound-speed profiles, bottom sound-speed gradients, bottom soundspeed and density fluctuations, and rough water/bottom interfaces. These data are then correlated with results from similar calculations without environmental variability but with attenuation as a free parameter used to maximize the correlation. By performing such comparisons over a band of frequencies, the effective frequency dependence of the bottom attenuation can be determined. The dominant environmental influences will then be identified and quantified.

\section{1:15}

3aUW12. Sparse acoustic gridding in an azimuthally complex environment. Erik R. Rike and Donald R. DelBalzo (Naval Res. Lab., Stennis Space Center, MS 39529)

A very sparse acoustic gridding method, the Objective Grid/Radials using Environmentally sensitive Selection (OGRES) algorithm, for sonar system performance predictions was reported for a realistic synthetic square annular ridge environment [E. R. Rike and D. R. DelBalzo, Proc. 6th ECUA (2002)] and a complex real-world environment in the Sea of Japan [E. R. Rike and D. R. DelBalzo, Proc. IEEE Oceans (2002)]. The sparse OGRES grids significantly outperformed uniform grids (matched for calculation time) in accuracy. Both cases were based on ground-truth uniform grids high in spatial density, but moderate-to-low in azimuthal density. The present study is a very sparse gridding of a realistic synthetic spiral ridge based on a ground-truth uniform grid high in both spatial and azimuthal density. This analysis extends the previous work by exploring the relationship between the spatial curvature of bathymetric features and optimal azimuthal grid density. The trade-offs between calculation time and accuracy of sonar system predictions for both very sparse and uniform grids are discussed. [Work supported by ONR/NRL under PE 62435N.] 
3aUW13. Acoustic modal analysis in an anisotropic internal wave field. Roger Oba and Steven Finette (Acoust. Div. Naval Res. Lab, Washington, DC 20375)

Simulations show that an anisotropic, internal wave field in shallow water can cause significant horizontal redirection of acoustic propagation, requiring true three-dimensional sound modeling. Calculations are presented for a sound speed profile that is perturbed by both a nonlinear internal wave packet, and a linear background internal wave field that is homogeneous and isotropic. A 3D parabolic equation code is used to propagate a $400 \mathrm{~Hz}$ signal through the spatially and temporally varying sound speed distribution. A single mode starter field at the source is propagated to a receiver location, and there the field is decomposed into modes to determine the degree of modal coupling. If the compressional wave number vector is perpendicular to that of the nonlinear internal wave packet wave number vector, the modeled acoustic field is nearly adiabatic and can be approximated using horizontal mode decomposition. In this case the pressure wave shows horizontal refraction, and ducting. For propagation paths not perpendicular to the internal wave number vector, the acoustic field can b enonadiabatic, and mode coupling can be significant. Modal analysis shows similar refraction and coupling results for lower frequencies. [Work supported by ONR.]

WEDNESDAY MORNING, 4 DECEMBER 2002
3aUW14. Simulations of time spreading in shallow water propagation. Eric I. Thorsos, W. T. Elam, Dajun Tang, Frank S. Henyey, Kevin L. Williams, and Stephen A. Reynolds (Appl. Phys. Lab., Univ. of Washington, 1013 NE 40th St., Seattle, WA 98105)

Pulse propagation in a shallow water wave guide leads to time spreading due to multipath effects. Results of PE simulations will be described for pulse propagation in shallow water with a rough sea surface and a flat sandy sea floor. The simulations illustrate that such time spreading may be significantly less at longer ranges than for the flat surface case. Pressure fields are simulated in two space dimensions and have been obtained using a wide-angle PE code developed by Rosenberg [A. D. Rosenberg, J. Acoust. Soc. Am. 105, 144-153 (1999)]. The effect of rough surface scattering is to cause acoustic energy initially propagating at relatively high angles but still below the critical angle at the sea floor to be eventually shifted to grazing angles above the critical angle. This energy is then lost into the bottom, effectively stripping higher propagating modes. The surviving energy at longer ranges is concentrated in the lowest modes and shows little effect of time spreading. Thus, the effect of rough surface scattering is found to produce a simpler temporal field structure than if the surface were treated as flat. [Work supported by ONR.]

MERIDA ROOM (HYATT), 9:00 TO 11:00 A.M.

\title{
Meeting of Accredited Standards Committee (ASC) S2 Mechanical Vibration and Shock
}

to be held jointly with the

ANSI-Accredited U.S. Technical Advisory Group (TAG) Meetings for:

ISO/TC 108 Mechanical Vibration and Shock

ISO/TC 108/SC 1 Balancing, including balancing machines

ISO/TC 108/SC 2 Measurement and evaluation of mechanical vibration and shock as applied to machines, vehicles and structures

ISO/TC 108/SC 3 Use and calibration of vibration and shock measuring instruments

ISO/TC 108/SC 5 Condition monitoring and diagnostics of machines and ISO/TC 108/SC 6 Vibration and shock generating systems

\author{
R. J. Peppin, Chair S2 \\ 5012 Macon Road, Rockville, Maryland 20852
}

D. J. Evans, Vice Chair S2 and Chair of the U.S. Technical Advisory Group (TAG) for ISO/TC 108 Mechanical Vibration and Shock and Chair of the U.S. Technical Advisory Group (TAG) for ISO/TC 108/SC 3 Use and calibration of vibration and shock measuring devices

National Institute of Standards and Technology (NIST), 100 Bureau Drive, Stop 8221, Gaithersburg, Maryland 20899-8221

R. Eshleman, Acting Chair of the U.S. Technical Advisory Group (TAG) for ISO/TC 108/SC 1 Balancing, including balancing machines

Vibration Institute, 6262 Kingery Highway, Ste. 212, Willowbrook, Illinois 60514

A. F. Kilcullen, Chair of the U.S. Technical Advisory Group (TAG) for ISO/TC 108/SC 2 Measurement and evaluation of mechanical vibration and shock as applied to machines, vehicles and structures

5012 Woods Road, Hedgesville, West Virginia 25427

R. Eshleman, Vice Chair of the U.S. Technical Advisory Group (TAG) for ISO/TC 108/SC 2 and Chair of the U.S. Technical Advisory Group (TAG) for ISO/TC 108/SC 5 Condition monitoring and diagnostics of machines

Vibration Institute, 6262 Kingery Highway, Ste. 212, Willowbrook, Illinois 60514

G. Booth, Chair of the U.S. Technical Advisory Group (TAG) for ISO/TC 108/SC 6 Vibration and shock generating systems 44 Bristol Street, Branford, Connecticut 06405-4842

Accredited Standards Committee S2 on Mechanical Vibration and Shock. Working group chairs will report on the status of various shock and vibration standards currently under development. Consideration will be given to new standards that might be needed over the next few years. There will be a report on the interface of S2 activities with those of ISO/TC 108 and its subcom- 
mittees including plans for future meetings of ISO/TC and/or its Subcommittees. The Technical Advisory Groups for ISO/TC 108 and the Subcommittees listed above consists of members of S2 and other persons not necessarily members of those Committees. Open discussion of committee reports is encouraged.

Scope of S2: Standards, specifications, methods of measurement and test, and terminology in fields of mechanical vibration and shock, and condition monitoring and diagnostics of machines, but excluding those aspects which pertain to biological safety, tolerance and comfort.

WEDNESDAY AFTERNOON, 4 DECEMBER 2002

GRAND CORAL 2, 1:15 TO 2:30 P.M.

\title{
Session 3pAA
}

\section{Architectural Acoustics: Halls, Theaters and Cathedrals}

\author{
David E. Marsh, Chair \\ Pelton Marsh Kinsella, 1420 West Mockingbird Lane, Suite 400, Dallas, Texas 75247-4932
}

Contributed Papers

$1: 15$

3pAA1. Virtual auditorium concepts for exhibition halls. Jack Evans, Chad Himmel, and Sarah Knight (JEAcoustics/Eng. Vib. Acoustics \& Noise Solutions, 5806 Mesa Dr., Ste. 380, Austin, TX 78731-3742)

Many communities lack good performance facilities for symphonic music, opera, dramatic and musical arts, but have basic convention, exhibition or assembly spaces. It should be possible to develop performance space environments within large multipurpose facilities that will accommodate production and presentation of dramatic arts. Concepts for moderate-cost, temporary enhancements that transform boxy spaces into more intimate, acoustically articulated venues will be presented. Acoustical criteria and design parameters will be discussed in the context of creating a virtual auditorium within the building envelope. Physical, economic, and logistical limitations affect implementation. Sound reinforcement system augmentation can supplement the room conversion. Acceptable control of reflection patterns, reverberation, and to some extent, ambient noise, may be achieved with an array of nonpermanent reflector and absorber elements. These elements can sculpture an enclosure to approach the shape and acoustic characteristics of an auditorium. Plan and section illustrations will be included.

\section{$1: 30$}

3pAA2. Municipal Theater of Lima: Reconstruction after fire. Jorge Moy, Mario Segami, Jose Ferrari (Universidad Peruana de Ciencias Aplicadas., Dep. de Arquitectura., Av Prolongacion Primavera 2390, Surco, Lima, Peru), and Richard A. Moscoso (Pontificia Universidad Catolica del Peru, Lima, Peru)

The Municipal Theater of Lima was inaugurated on 28 July 1920 as the Forero Theater and was acquired by the Metropolitan Municipality of Lima in 1929. On 2 August 1998 it was partially destroyed by fire. The damage to the audience area was minimal, allowing complete restoration. However, the stage was destroyed, which required a complete reconstruction. The Municipality of Lima launched a project competition for its reconstruction and restoration the results of which were known in 2001. One of the objectives was the correction of well-known acoustical defects, which had been never solved. The results of computer simulations of the original theater with a commercially available ray-tracing-type computer program will be presented, showing its acoustical evaluation and available options to correct the acoustical defects. The acquisition of five lots around the theater site will allow increases in the fly tower, the dressing rooms, the pit, and the shops. There are plans for the modernization of the technical and mechanical services, the accesses, the air-conditioning sys- tem, and security as well. These modifications will allow all types of presentations of contemporary arts. Retaking its position as first in the national scene, the Municipal Theater will be able to house sophisticated national and international performances.

\section{1:45}

3pAA3. Reuse of a cylinder shape lecture hall as a recital hall. Weihwa Chiang, Weiping Wu, Wenling Chih, and Choye Lee (Natl. Taiwan Univ. of Sci. and Technol., 43, Sec. 4, Keelung Rd., Taipei, Taiwan)

A cylinder shape lecture hall in Danchiang University, Taiwan was reused as a 300 -seat recital hall with recording facilities. Acoustical design was analyzed with computer models and a scale model. Besides the acoustical defects due to the cylinder plan, the low ceiling height of $3.3 \mathrm{~m}$ caused a low reverberation with ceiling preserved but poor sound propagation with the ceiling removed because of the 1.2 deep girders. Three major convex-curved walls were used, two on both sides of the stage and the other as the partition between the hall and the control room. Two side boxes on each side of the hall were used with the box facing slightly tilted down to provide lateral reflection. Installing oval shaped supply ducts diffused the remaining concave wall surfaces. Triangular overhead panels in various sizes were used to diffuse first order ceiling reflection. Scale modeling demonstrated that most of the design strategies were not only effective in enhancing uniform energy distribution spatially but also randomized the reflection pattern for individual receivers. A low strength factor of $11 \mathrm{~dB}$ was beneficial for large chamber groups. On going study is conducted regarding the field tuning and measurements of the hall.

\section{2:00}

3pAA4. Acoustic conditioning of the metropolitan cathedral of Porto Alegre, RS, Brazil. Flavio M. Simoes, Luciano B. Nabinger, and Aline I. Ramalho (Curso Arquiteura Urbanismo-Ritter dos Reis, FAIR, R. Orfanotrofio, 555 Porto Alegre, Brazil)

In the acoustic study of the Metropolitan Cathedral of Porto Alegre, RS, Brazil, initially background noise and reverberation time were measured. A digital model was built using acoustic simulation software AcustaCadd, applying the values of the measured reverberation time. Then reverberation time, speech intelligibility, and geometric acoustics were analyzed. As a result the Project of Acoustic Conditioning was developed to correct the high reverberation time, by increasing absorption with the installation of $65000 \mathrm{~m}$ of panels of glass wool $(100 \mathrm{~mm}, 60 \mathrm{~kg} / \mathrm{m})$. Advantage was taken of existing details in the plaster to embed the panels in the walls. Also the volume of the choir and of the lateral balcony to the 
altar was reduced and the interior of this was covered with the same glass wool. Special care was taken to minimize alterations to the architectural characteristics of the place, because it is a construction of historical importance. The measured values of background noise were also analyzed and appropriate acoustic isolation considered. The final measure of the reverberation time showed an average reduction of 5 seconds and better speech intelligibility, long demanded by the users. [Work supported by FAIR/FUNDATEC, BR; IUCC-US, SP.]

\section{2:15}

3pAA5. Subjective evaluation of a concert hall's acoustics using a free-format-type questionnaire and comparison with objective measurements. Toshiyuki Okano (Takenaka Res. and Development Inst., 1-5-1, Ohtsuka, Inzai, Chiba 270-1395, Japan, okano.toshiyuki@takenaka.co.jp) and Leo L. Beranek (Cambridge, MA 02138)

A free-format type of audiences' judgment of the acoustical properties of a hall and music critics' writings were used as the basis for this study.
These subjective responses are related to the Dai-Ichi Seimei Hall in Tokyo. This hall is an oval-shaped, one-balcony space, seating 767 persons. Its primary use is for various types of chamber music and solo-instrument performances. Eight acoustical attributes were investigated, "reverberation," "clarity," "loudness," "intimacy," "spaciousness," "balance," "localization," and "timbre," plus "general impression." Subjective comments about these attributes were obtained. Objective measurements were made in the hall and are compared with those made in several similarsized halls of two shapes. In the rear seats of two oval-shaped halls the strength factor GE (determined in the first $80 \mathrm{~ms}$ of the impulse response) was greater than the GE found in the rear seats of similar-sized rectangular halls. The subjective results and the objective measurements were closely correlated, especially for reverberation, clarity, and warmth (a subcomponent of timbre). It was suggested that the greater strength GE in the rear seats made the hall seem smaller and thus more intimate. The subjective comments also confirmed the hall's wide applicability, indicating that the acoustical characteristics used for its design were well chosen.

WEDNESDAY AFTERNOON, 4 DECEMBER 2002

CORAL KINGDOM 2 AND 3, 1:15 TO 3:05 P.M.

\title{
Session 3pAB
}

\section{Animal Bioacoustics and Psychological and Physiological Acoustics: Electrophysiological Investigations of Animals II}

\author{
Eduardo Mercado III, Chair \\ Center for Molecular and Behavioral Neuroscience, Rutgers University, 197 University Avenue, Newark, New Jersey 07102
}

Invited Papers

1:15

3pAB1. Evoked-potential study of echolocation in whales and dolphins. Alexander Ya. Supin (Inst. of Ecology and Evolution, 33 Leninsky Prosp., 119071 Moscow, Russia), Paul E. Nachtigall, and Whitlow W. L. Au (Univ. of Hawaii, Kailua, HI)

During echolocation, whales and dolphins perceive faint echoes very shortly after loud ongoing pulses. How does the auditory system avoid the masking of the echo by the preceding pulse? To investigate mechanisms of release from masking, auditory brainstem evoked responses $(\mathrm{ABR})$ were recorded in bottlenose dolphins and a false killer whale both during active echolocation and during paired external acoustic stimulation. When the animal ensonified a target, both the ongoing pulse and echo (2-ms delay) evoked ABRs of comparable amplitudes, in spite of echo intensity of $40 \mathrm{~dB}$ relative the ongoing pulse near the animal's head. When a phantom echo was used (emitted pulses were replayed through an electronic channel with the same delay of $2 \mathrm{~ms}$ ), the ABR to the echo was comparable to that to the emitted pulse at the echo intensity of $20 \mathrm{~dB}$, but the ABR decreased when the echo intensity diminished to $40 \mathrm{~dB}$ relative to the ongoing pulse. An explanation of higher efficiency of the real than the phantom echo may be a difference between spectra of the real echo and the outgoing pulse. Paired-pulse stimulation confirmed that a moderate spectral difference between two pulses resulted in release from masking at short interpulse intervals.

\section{$1: 35$}

3pAB2. Frequency and directional selectivity in primary auditory afferents in fishes. Richard R. Fay (Parmly Hearing Inst., Loyola Univ. of Chicago, 6525 N. Sheridan Rd., Chicago, IL 60626, rfay@luc.edu)

Coding of frequency and particle motion directionality is described for two fishes; goldfish (a sound-pressure specialist), and oyster toadfish (a hearing generalist). The goldfish hears in a wide bandwidth $(>2 \mathrm{kHz})$ and the toadfish in a narrower one $(<400 \mathrm{~Hz})$. Nevertheless, the mechanisms and degree of peripheral frequency analysis appear to be similar. Reverse correlation (revcor) studies of frequency filtering in the auditory nerve reveal two fundamental filter types in both species; one at low and one at higher frequencies. The details of the two filter functions are similar, and one can be transformed into the other by introducing a simple spectral tilt to one of the filters. This suggests that only one fundamental filter exists for each species, and that the second filter type may be derived from it through alterations in the mode of coupling between the hair cell cilia and restraint structures. In spite of this simple filtering, goldfish effectively analyze the frequency components in complex and simple sounds. In both species, primary afferents show directional preferences in 3-D space that correspond to the directionalities of hair cells. Frequency analysis and directional processing in fishes share many fundamental features with those of most other vertebrates. 
3pAB3. Auditory brainstem response (ABR), a noninvasive electrophysiological method for the study of hearing ability of fishes. Hong Yan (School of Biological Sci., Univ. of Kentucky, Lexington, KY 40506-0225)

Over the past $100+$ years several methods have been used to study hearing ability of fish. Behavioral (psychophysical) methods including food reward, barrier crossing, and electrical shock conditioning are used to train fish to respond to sound. Invasive-type of electrophysiological methods include single unit and microphonics recordings. In 1996, my laboratory developed auditory brainstem response (ABR) recording technique, an electrophysiological far-field recording method, to record acoustically evoked brainstem potentials from fish. This noninvasive method overcomes the major limitations of traditional behavioral (prolonged training period; erratic behavioral response) and electrophysiological (invasive surgery) methods. The ABR recording can be achieved easily with assemblage of conventional electrophysiological devices with the aid of computer programs. Since the development of this noninvasive electrophysiological recording method, it has been widely adopted by many laboratories around the world to study fish hearing. The role of gasbladder in hearing enhancement, the ontogenetic development of hearing ability of embryo and larval fish, the impacts of noise exposure on threshold shifts, the effect of aging on hearing all have been understood with the use of ABR method. This quick pace of achievement cannot be obtained without the use of this noninvasive recording technique. [Work supported by NIH, NOHR, IMLS.]

2:15

3pAB4. Sea turtle auditory behavior. Martin Lenhardt (Prog. in Biomed. Eng., Virginia Commonwealth Univ., Box 168 MCV, Richmond, VA 23298-0168)

Little is known of sea turtles' auditory behavior. Startles (neck contractions) were observed in 25 loggerheads (Caretta caretta) to tones from 35 to $1000 \mathrm{~Hz}$, when the turtles were near the bottom of holding tanks at a depth of 1 meter. A composite audiogram revealed lowest thresholds in the 400-500 Hz range (106 dB SPL re $1 \mu \mathrm{m})$. Thresholds at 735 and $1000 \mathrm{~Hz}$ were 117 and $156 \mathrm{~dB}$, respectively. Thresholds in the $100-200 \mathrm{~Hz}$ range were $\sim 124 \mathrm{~dB}$, with lower frequencies being $10-12 \mathrm{~dB}$ higher. Tank diving behavior was elicited with $30 \mathrm{~Hz}$ at $164 \mathrm{~dB}$. ABR thresholds to vibration clicks with peak energy at $500 \mathrm{~Hz}$ were $113 \mathrm{~dB}$. Seismic air guns (Bolt 600) were employed in a large net enclosure. Turtles increased swimming speed for exposures in the 151-161 dB levels. Avoidance $\sim 175 \mathrm{~dB}$ was common in initial trials, before habituation. ABRs pre- and post-air gun exposures revealed TTS of more than $15 \mathrm{~dB}$ in one animal with recovery in 2 weeks. Air guns in depths $>10 \mathrm{~m}$ may result in more energy in the low frequencies with unknown bioeffects. Turtle repelling with sound is possible and can be made practical (Lenhardt, US Patent No. 6388949).

\section{Contributed Papers}

2:35

3pAB5. Some constraints on the use of ABR in hearing studies: Investigation in dolphins. Vladimir Popov and Alexander Supin (Inst. of Ecology and Evolution, Russian Acad. of Sci., 33 Leninsky Prosp., 119071 Moscow, Russia)

The main difficulty in using ABR in frequency-tuning measurements arises from the fact that these responses are evoked only by transient acoustic stimuli. This limits their use as probes for measuring frequency selectivity. Even with a slowly rising and falling stimulus, only a shorter part of the stimulus may be effective to evoke the response, and the spectrum of this short part is broader than the spectrum of the whole burst. It was shown in tone-tone masking study in dolphins, that tuning curves at lower frequencies reflect not the real frequency tuning but broad spectral bandwidth of the probe's effective part. In the bottlenose dolphin, this effective part is not longer than $0.5 \mathrm{~ms}$, irrespective of the actual probe duration. Another problem in the use of the ABR technique is that it is not clear which spectral part of the wideband stimulus participates in $A B R$ generation, and what is the contribution of various parts of the cochlear partition to ABR. ABR to clicks and noise bursts of various frequency bands were recorded in dolphins in order to find these contributions. ABR amplitude increased with increasing the stimulus frequency, thus indicating a higher contribution of basal cochlear parts.
2:50

3pAB6. Experience-dependent cortical processing of complex sounds by rats. Itzel Orduna, Mark A. Gluck (CMBN, Rutgers Univ., 197 University Ave., Newark, NJ 07102, orduna@axon.rutgers.edu), Eduardo MercadoIII (SUNY at Buffalo, Buffalo, NY 14260), Jennifer F. Linden, Shaowen Bao, Michael M. Merzenich (UCSF, San Francisco, CA 94143), and Maneesh Sahani (UCL, London, UK)

Auditory cortex is thought to play a critical role in the processing of species-specific vocalizations and other acoustically complex sounds. Although evolutionary processes strongly constrain cortical sensitivities to sound, cortical processing is not fixed by biology, but rather is shaped by the auditory experiences of each individual. Auditory cortical neurons in adult rats respond selectively to spectrotemporal features of complex sounds. These selective responses are predictive of rats' behaviorally measured perceptual sensitivities. With extensive training, the abilities of rats to discriminate frequency-modulated sounds improve. Recordings from cortical neurons in trained rats show increased sensitivities to features of the sounds used in training. These results demonstrate that discrimination training with biologically irrelevant complex sounds can change how cortical neurons process those sounds. Changes in cortical processing of complex sounds can also be induced by controlling activity in neuromodulatory neurons while a rat experiences the sounds. Pairing the presentation of complex sounds with electrical stimulation of basal forebrain neurons radically changes cortical sensitivities to spectrotemporal features of those sounds. These data suggest that auditory cortical representations of acoustic events can be flexibly adjusted throughout the lifespan of an individual rate. Presumably, similar or greater flexibility exists in animals with more sophisticated auditory systems. 


\title{
Session 3pBB
}

\section{Biomedical Ultrasound/Bioresponse to Vibration: Lithotripsy II}

\author{
Pei Zhong, Chair \\ Department of Mechanical Engineering and Materials Science, Duke University, 1 Science Drive, Box 90300, \\ Durham, North Carolina 27708
}

Contributed Papers

\section{1:00}

3pBB1. A gypsum-based artificial stone for shock wave lithotripsy research. James A. McAteer, James C. Williams, Jr., Andrew P. Evan (Dept. of Anatomy \& Cell Biol., Indiana Univ. School of Medicine, 635 Barnhill Dr., Indianapolis, IN 46202-5120, mcateer@anatomy.iupui.edu), Robin O. Cleveland (Boston Univ., Boston, MA 02115), Michael R. Bailey, and Lawrence A. Crum (Univ. of Washington, Seattle, WA 98105)

Natural kidney stones are heterogeneous in structure, composition, material properties and fragility, and as such are problematic for use in determining the mechanisms of SW-action in SWL. A variety of model stones have been developed. We have adopted Ultracal-30 gypsum [Dahake and Gracewski, J. Acoust. Soc. Am. 102, 2138 (1997)] for in-vitro and in-vivo studies. U-30 stones $(7.5 \times 6.5 \mathrm{~mm})$ cast in polystyrene molds were liberated with chloroform and stored in water. Drop-impact testing of dry stones showed a linear relationship between increase in surface area of fragments and energy applied. Breakage of hydrated stones in a researchelectrohydraulic lithotripter, likewise showed a linear increase in fragment area with increased SW-number and SW-voltage. The density (1800 $\left.\mathrm{kg} / \mathrm{m}^{3}\right)$ and transverse $(1520 \mathrm{~m} / \mathrm{s})$ and longitudinal $(3100 \mathrm{~m} / \mathrm{s})$ wave speeds of U-30 stones place them in the range determined for natural stones. U-30 stones implanted in pig kidneys exhibited cavitation erosion and spall fracture similar to stones in-vitro, and U-30 stones in-vitro and in-vivo showed equivalent response to SW-rate (200\% higher fragmentation at $0.5 \mathrm{~Hz}$ compared to $2 \mathrm{~Hz}$ ). U-30 stones softened with prolonged exposure to water and degraded during long-term implantation in-vivo. With these cavaets U-30 stones provide a useful model for SWL research. [Work supported by NIH P01-DK43881.]

\section{$1: 15$}

3pBB2. Effect of the diameter and the sound speed of a kidney stone on the acoustic field induced by shock waves. Robin Cleveland and Juan Tello (Dept. of Aerosp. and Mech. Eng., Boston Univ., Boston, MA 02215)

A time domain finite difference solution to the acoustic wave equation was used to model the propagation of lithotripsy shock waves in kidney stones. The stones were modeled as cylindrical objects and the impact of stone sound speed, stone diameter, and the internal structure on the pressure field inside the stones was calculated. The sound speed was varied from 2500 to $3500 \mathrm{~m} / \mathrm{s}$ and had a minimal effect on the peak pressures within the stone. However, reducing the stone diameter from $15 \mathrm{~mm}$ to 3 $\mathrm{mm}$ reduced the peak negative pressure by $67 \%$. For cases where the sound speed of the stone was inhomogeneous (inner core different from outer ring), the amplitudes of the peak pressures varied slightly, however the spatial distribution of the peak negative pressure varied significantly. In particular configurations with a lower outer sound speed lead to the peak negative pressure occurring at the boundary between the layers. The results indicate that the pressure field in a kidney stone is very sensitive to the size and internal composition of a kidney stone. This effect could be partly responsible for the large variance in fragility observed in human stones. [Work supported by the Whitaker Foundation and NIH-DDK.]

$1: 30$

3pBB3. Progression of crack formation in artificial kidney stones subject to shock waves. Javier Van Cauwelaert and Robin Cleveland (Aerosp. and Mech. Eng. Dept., Boston Univ., 110 Cummington St., Boston, MA 02215)

We used micro computed tomography (CT) imaging to follow the progressive development of cracks in artificial kidney stones. The artificial stones were made from U30 cement with a cylindrical shape $(6.5 \mathrm{~mm}$ diameter and $7.5 \mathrm{~mm}$ long). The stones were held within a polypropylene vial in one of three different orientations: vertical, horizontal, and angled at $45 \mathrm{deg}$. The stones were treated with an electromagnetic lithotripter using between 50 and 150 shock waves. The initiation and growth of cracks was observed using microCT. We found that crack formation in the U30 stones was influenced by the orientation of the stone with respect to the shock wave (SW) propagation direction. Vertical stones developed a spall-like crack near the distal surface; horizontal stones had little internal damage for the number of shock waves applied; and angled stones were damaged primarily in the vicinity of the leading corner. The position of the cracks were in qualitative agreement with the numerical solutions of the pressure field inside the stones. The elastic properties of the U30 stones are being measured which will alow a quantitative analysis of crack growth to be performed and compared to the experimental data. [Work supported by the Whitaker Foundation.] 
3pBB4. Role of cavitation in stone fragmentation by shock wave lithotripsy. James A. McAteer, James C. Williams, Jr., Andrew P. Evan (Dept. of Anatomy \& Cell Biol., Indiana Univ. School of Medicine, 635 Barnhill Dr., Indianapolis, IN 46202-5120, mcateer@anatomy.iupui.edu), Robin O. Cleveland (Boston Univ., Boston, MA 02115), Michael R. Bailey, and Lawrence A. Crum (Univ. of Washington, Seattle, WA 98105)

Several strategies were used to assess the importance of cavitation in the breakage of stones by an electrohydraulic lithotripter in vitro. (1) Stones exposed to SWs at atmospheric pressure broke readily. However, stones treated at high overpressure $(\mathrm{OP} \sim 125 \mathrm{~atm})$ sufficient to eliminate cavitation did not break into fragments even with twice the number of SWs. Stones at OP did, however, develop transverse fractures typical of spall. This suggests that cavitation contributes to stone fragmentation, but is clearly not the only mechanism involved in stone breakage. (2) Cylindrical model stones positioned vertically in the acoustic field of a researchEHL showed proximal erosion and spall. However, placement of a mylar disk against the flat leading face of the stone eliminated cavitation-erosion, and spall did not occur. This suggests that cavitation may contribute to stone fracture by spall. (3) Time reversal of the lithotripter wave form using a pressure release reflector (Prel) also prevented stone fragmentation. With the Prel insert the tensile phase of the SW preceding the compressive wave bubble growth is interrupted by $\mathrm{P}+$ and, thus, cavitation is suppressed. Together, these results suggest that cavitation plays an important role in the breakage of stones by lithotripter shock waves. [Work supported by NIH P01-DK43881.]

\section{2:00}

3pBB5. Evidence of oxidative stress in both kidneys after shock-wave lithotripsy to one renal pole. Lynn R. Willis, James E. Klaunig, Yong $\mathrm{Xu}$ (Dept. of Pharmacology and Toxicology, Indiana Univ. School of Medicine, 635 Barnhill Dr., Indianapolis, IN 46202), Bret A. Connors, Andrew P. Evan (Indiana Univ. School of Medicine, Indianapolis, IN 46202), and James E. Lingeman (Methodist-Clarian Hospital, Indianapolis, IN 46202)

Shock-wave lithotripsy (SWL) damages renal tissue and reduces renal perfusion. These studies asked whether SW-induced injury or reduced perfusion might cause oxidative stress. Two groups of 3 anesthetized pigs received 2000 shock waves (SWs) at $24 \mathrm{kV}$ (Dornier HM3, $2 \mathrm{~Hz}$ ) to one renal pole. Renal hemodynamics were measured $1 \mathrm{~h}$ before and 1 and $4 \mathrm{~h}$ after SWL. Samples of renal tissue from upper and lower poles of each kidney were analyzed for malonaldehyde (lipid peroxidation) and 8-OHdeoxyguanosine (OH8dG, DNA damage). SWL reduced perfusion in both kidneys, but injured only the shocked kidneys. Malonaldehyde levels were elevated in both poles of the shocked kidneys, but were not elevated in the contralateral unshocked kidneys. In contrast, OH8dG levels were elevated in the treated pole of the shocked kidneys, and in both poles of the contralateral unshocked kidneys. In conclusion, (1) The elevated levels of malonaldehyde seen in the shocked kidneys but not in the unshocked kidneys are consistent with lipid peroxidation occurring after SWLinduced tissue injury; (2) elevated OH8dG levels in contralateral unshocked kidneys denotes oxidative stress and may reflect reperfusion injury associated with SWL-induced renal vasoconstriction. [Work supported by a grant from NIH P01 DK43881.]
3pBB6. The reduced renal blood flow observed after exposure to shock wave lithotripsy involves intact renal nerves. Bret A. Connors, Andrew P. Evan, Lynn R. Willis, Jay R. Simon (Indiana Univ. School of Medicine, Indianapolis, IN 46202, connors@ anatomy.iupui.edu), Arieh L. Shalhav, Ryan F. Paterson, Ramsay L. Kuo, and James E. Lingeman (Methodist Hospital Inst. for Kidney Stone Disease, Indianapolis, IN 46202)

Treating one kidney with high-energy acoustic shock waves reduces blood flow $65 \%$ in that kidney and 33\% in the contralateral unshocked kidney. We tested the hypothesis that renal nerves were involved in this response. Six-week old pigs underwent unilateral renal denervation. Nerves along the renal artery of one kidney were cut and the artery was painted with $10 \%$ phenol. After 2 weeks, the pigs were anesthetized and bilateral renal function was determined using inulin and PAH (paraaminohippurate) clearance. Glomerular filtration rate (GFR, inulin clearance) and renal blood flow (PAH clearance) were measured both before and after lithotripsy to the lower pole of the innervated kidney (2000 shocks, $24 \mathrm{kV}$, unmodified Dornier HM-3) or sham lithotripsy. Both kidneys were then removed to measure norepinephrine content in the tissue. Norepinephrine levels were significantly reduced (more than 90\%) in denervated kidneys indicating complete denervation. As expected, blood flow and GFR fell (approximately 50\%) after lithotripsy in the innervated shocked kidneys, but did not fall significantly in denervated contralateral kidneys or in sham lithotripsy animals. Results suggest that renal nerves mediate the fall in contralateral renal function after lithotripsy. [Work supported by NIH P01 DK43881.]

\section{$2: 30$}

3pBB7. Effects of the viscosity of the liquid in the microcapsule on deformation process of a bubble for developing DDS using shock waves. Masaaki Tamagawa, Ichiroh Yamanoi, and Toshiaki Matsuo (Grad. School of Life and Systems Eng., Kyushu Inst. of Technol., Wakamatsu-ku, Fukuoka 808-0196, Japan, tama@life.kyutech.ac.jp)

This paper describes the fundamental investigations for developing new DDS using shock waves and bubbles, especially effects of the viscosity of the liquid in the capsules on the deformation process of the bubbles. The viscosity of the liquid in the microcapsules requires controlling the bubble position and motion, deformation process to optimize the disintegration of the capsules. Using plane shock wave in the water by shock tube apparatus, a bubble near the curved gelatin wall was observed by high-speed framing camera. The deformation process for a bubble was analyzed by image processing. From these experiments, it is found that the viscosity of the liquid around the bubble suppress the amplitude of the bubble oscillations and collapsing the bubble. This means that the viscosity of the liquid in the capsule should be as small as possible for developing DDS using shock waves. In spite of this result, the effects of curvature and elasticity of the wall on the deformation process is almost the same as low viscosity. [Work supported by a Grant-in-Aid for Scientific Research from the Ministry of Education, Science, Sports and Culture, Japan.] 


\title{
Session 3pMU
}

\section{Musical Acoustics: Hands-On Explorations in Acoustics}

\author{
James P. Cottingham, Cochair \\ Physics Department, Coe College, Cedar Rapids, Iowa 52402 \\ Leonardo Fuks, Cochair \\ Escola de Musica, Universidade do Brazil UFRJ, Rue do Passero 98, Rio de Janeiro 20021-290, Brazil
}

Hands-on activities in acoustics, open to all meeting attenders, but designated primarily

for accompanying persons, both adults and children

WEDNESDAY AFTERNOON, 4 DECEMBER 2002

CORAL KINGDOM 1, 1:00 TO 2:35 P.M.

\section{Session 3pNS}

\author{
Noise: Hearing Protection II \\ Daniel P. Salomon, Cochair \\ Comaudi, Patriotismo 706, Colonia Mixcoac 03730, D.F. Mexico \\ Elliott H. Berger, Cochair \\ EAR/Aearo Company, 7911 Zionsville Road, Indianapolis, Indiana 46268-1657 \\ Chair's Introduction-1:00 \\ Contributed Papers
}

1:05

3pNS1. Current status of standards for testing electroacoustic hearing protectors. William J. Murphy and John R. Franks (NIOSH Hearing Loss Prevention Section, 4676 Columbia Pkwy., M.S. C-27, Cincinnati, OH 45226-1998,wjm4@cdc.gov)

Electroacoustic hearing protectors encompass several classes of electronics: level limiting, amplitude compression and active noise control (ANC) devices. Each class of protector seeks to enhance performance by overcoming insertion loss and/or improving low-frequency attenuation. Level-limiting devices turn off the amplification when the external sound level exceeds a preset threshold. Amplitude compression devices apply variable gain and limit amplification of signals above the threshold. ANC devices create an out-of-phase signal under the protector to improve the low-frequency attenuation of earmuffs. ANC devices typically perform best in continuous noise that has strong harmonic components that can be predicted and cancelled. The different methods of enhancing protector performance require separate standards to understand both the static and dynamic performance. Testing of ANC devices has further requirements which require knowledge of both passive and active electronic conditions and in noise with different crest factors. This talk will review a variety of tests of the different protector classes.

$1: 20$

3pNS2. Objective measurements for the assessment of hearing protectors attenuation at high level impulsive noise. Felipe Vergara, Samir N. Y. Gerges, Washington J. N. de Lima (Federal Univ. of Santa Catarina, Mech. Eng., Cx.P. 476, Florianopolis, SC, Brazil, CEP:88040-900), and Robert Birch (Univ. of Liverpool, Liverpool L69 $3 \mathrm{H}, \mathrm{UK})$

The evaluation of hearing protectors for the attenuation of high amplitude impulsive noise cannot be carried out using the conventional subjective Real Ear Attenuation at Threshold (REAT) technique. In the case for high level impulsive noise it is not possible to conduct subjective type tests with volunteer listeners, therefore, other methods based on using 
artificial human head forms need to be considered. In this paper we present an objective technique that uses an artificial head, ear-canal simulator and shock tube to assess earplug and earmuff protectors. The shock tube is used as a means of producing controlled repeatable high amplitude pressure pulses $(>140 \mathrm{~dB})$ of varying rise time, amplitude, and duration. The artificial head and ear simulator with a protector are mounted inside the shock tube and subjected to a high level sound pulse. Pressure levels are measured simultaneously outside of the protector (incident pulse) and inside at the ear simulator at the eardrum position. The attenuation of peak pressure levels in the time domain and the corresponding spectra of the pulses are determined. The technique offers pointers towards future standards of performance hearing protectors for applications with high level impulsive noise. ation of the DH-132A Combat Vehicle Crewmans helmet (CVC) when used in conjunction with CEP (measured in accordance with ANSI S12.61997 ) is reported with an NRR of $27 \mathrm{~dB}$ vs $15 \mathrm{~dB}$ for the CVC alone. CEPs were installed in the helmets of 77 tracked-vehicle crewmembers. Following a 6-month trial period, crewmembers $(n=36)$ reported increased noise attenuation, improved speech communication, and reduced stress when using the CVC-CEP integration. The CEP is a cost-effective alternative to expensive active noise reduction helmets currently being fielded for use by tracked-vehicle crews. [Work supported in part by USACHPPM.]

\section{$1: 35$}

3pNS3. Attenuation of high-level acoustic impulses by hearing protectors. Jan Zera (Central Inst. for Labour Protection, Czerniakowska 16, 00-701 Warsaw, Poland)

Attenuation of acoustic impulses by hearing protectors was measured in peak level ranges of $115-135 \mathrm{~dB}$ and $145-170 \mathrm{~dB}$. The impulses were generated by a loudspeaker system in the low range of levels and by a blast of air expanding from a cylinder in the high level range. Transmission loss method was used to determine the difference between the peak level under the earmuff and outside the earmuff for over 30 different types of earmuffs. The measurements confirm that attenuation of high-level impulses depends on their peak level. In the $115-135 \mathrm{~dB}$ range the difference between the peak level outside and under the earmuff is approximately constant. In the $145-170 \mathrm{~dB}$ range, as the impulse level is increased, the level under the earmuff increases to a lesser extent. The peak-to-peak level characteristics determined for the 115 to $170 \mathrm{~dB}$ level range substantially differ for various types of protectors and may be a useful indicator of the effectiveness of protectors for impulse noise. [Work supported by the State Committee for Scientific Research Grants Nos. III-7.03 and III-6.07.]

\section{1:50}

3pNS4. Communications earplug performance in tracked-vehicle crews. William A. Ahroon, Dale A. Ostler (U.S. Army Aeromedical Res. Lab., P.O. Box 620577, Fort Rucker, AL 36362-0577), Ben T. Mozo (Commun. \& Ear Protection, Inc., Enterprise, AL 36331-1174), and Cynthia M. Crossley (Martin Army Community Hospital, Fort Benning, GA 31905)

Tracked-vehicle crewmembers operate in an acoustical environment that is one of the most inhospitable encountered in the U.S. Army. Overall noise levels in the M1A2 Abrams tank and M2A2 Bradley Fighting Vehicle can be well over $100 \mathrm{dBA}$ and can exceed $130 \mathrm{~dB}$ in some octave bands. Due to the nature of the crewmembers' duties, speech communication is essential for effective operations and this capability is seriously degraded because of the nature of the noise environment. The Communications Earplug (CEP), consisting of miniature receivers attached to replaceable foam earplugs, is used in Army aviation to provide superior noise attenuation with improved speech communication. The noise attenu-
2:05

3pNS5. System for measurement of headband force in hearing protection devices and audiometric equipment. Osvaldo LlamasLlamas and Jose-Noe Razo-Razo (Centro Nacional de Metrologia, Div. de Vibraciones y Acustica, Carr. a Los Cues km 4,5 76241 El Marques, Qro., Mexico,ollamas@cenam.mx)

Application force influences audiometric results and hearing protection devices (HPD) attenuation performance. In HPD attenuation testing it is neccessary to know the application force. Audiometric studies are being conducted with standardized values of the application force. Design and results of a measurement device for bands and headbands application force are presented. Error analysis in the range from $1 \mathrm{~N}$ to $30 \mathrm{~N}$ is carried out and the associated measurement uncertainty is estimated. The device provides settings for test distances from $70 \mathrm{~mm}$ to $140 \mathrm{~mm}$ (vertical axis), and $115 \mathrm{~mm}$ to $195 \mathrm{~mm}$ (horizontal axis), as required in most of the available standards related with HPD and audiometric equipment.

2:20

3pNS6. Overpressure and noise due to multiple airbag systems in a passenger car. Robert Hickling (Sonometrics, Inc., 8306 Huntington Rd., Huntington Woods, MI 48070, sonomet@aol.com), Peter J. Henning, and Gary Newton, Jr. (Bruel \& Kjaer Sound and Vib. Measurement A/S, Livonia, MI 48154)

Multiple airbag systems in passenger cars can generate overpressure and noise that may be hazardous to human hearing. Overpressure is compression of the air inside a closed compartment caused by deployment of the bags. Noise results from the action of the gas inflating the bags. SAE J247 provides a standard for measuring the combination of overpressure and noise in a passenger compartment. A special microphone has recently been developed that meets this standard, which operates down to a fraction of a hertz. Details of the microphone are given. Little appears to have been published on the overpressure and noise of modern multiple airbag systems, but early results [R. Hickling, "The noise of the automotive safety air cushion," Noise Control Eng., May-June, 110-121 (1976)] provide a basic understanding of the phenomenon. Spectral data shows that peak overpressure occurs at about 2 to $3 \mathrm{~Hz}$. A significant reduction in overpressure and noise can be achieved with an aspirating airbag, originally developed at General Motors, whose outer structure is inflated with gas from the inflator, and whose inner structure draws in air from the passenger compartment through one-way cloth valves. Tests have shown that such bags function well when impacted. 


\title{
Session 3pPA
}

\section{Physical Acoustics and Signal Processing in Acoustics: The Coda and Other Stochastic Seismic Signals II}

\author{
Joseph A. Turner, Chair \\ Department of Engineering Mechanics, University of Nebraska-Lincoln, Lincoln, Nebraska 68588
}

Invited Papers

\begin{abstract}
1:00
3pPA1. Diffusing acoustic wave spectroscopy. John H. Page, Michael L. Cowan (Dept. of Phys. and Astron., Univ. of Manitoba, Winnipeg, MB R3T 2N2, Canada), and David A. Weitz (Dept. of Phys. and DEAS, Harvard Univ., Cambridge, MA 02138)

Diffusing Acoustic Wave Spectroscopy (DAWS) is an ultrasonic technique that has been developed to measure the dynamics of heterogeneous media from the temporal fluctuations of multiply scattered waves. This technique is similar to more recent developments in field fluctuation spectroscopy, called coda wave interferometry, that use variations in the seismic coda to infer changes in the medium with time. After reviewing the basic principles on which Diffusing Acoustic Wave Spectroscopy is based [M. L. Cowan, J. H. Page, and D. A. Weitz, Phys. Rev. Lett. 85, 453 (2000); Phys. Rev. E 65, 066605 (2002)], its potential as a sensitive method for probing the dynamics of strongly scattering materials will be illustrated with recent experiments on fluidized suspensions of particles. In this type of system, DAWS measures the local relative motion of the scatterers (or strain rate) on a length scale determined by the transport mean free path of the multiply scattered waves. When combined with the complementary technique of Dynamic Sound Scattering using singly scattered waves, DAWS can also determine the instantaneous velocity correlation length of the moving scatterers, thus giving a quite complete picture of the system dynamics over a wide range of length and time scales.
\end{abstract}

$1: 30$

3pPA2. Correlations in the seismic coda and elastic Green's functions. Michel Campillo and Anne Paul (LGIT UJF-CNRS BP53 38041, Grenoble, France)

The conjecture that the correlation of coda records at two stations may provide the Green's function between these two stations, or an approximation of it, is discussed. Under the assumption of equipartition and/or considering that we use a set of sources that is evenly diffracted in the whole space, it can be shown that the average cross correlation between the records of every earthquake at the two stations is an approximation of the Green's function between the two stations. Records from stations of the Mexican network are used to test this idea. The stack of the cross correlations indicates that a low-frequency coherent signal is present with a signal-to-noise ratio that was $\sim 0.2$ for a single correlation. The Green's function between two points at the surface is expected to be widely dominated by the Rayleigh wave. The signal that we extracted presents the characteristics expected for a Rayleigh wave: elliptical polarization in the radial-vertical plane and adequate group velocity. The coherent signal extracted from coda correlations has the symmetry properties of the Green tensor. In spite of the a priori limitations of this approach in seismology, the preliminary results presented here demonstrate its potential.

2:00

3pPA3. Coda wave interferometry, a new method for monitoring change. Roel Snieder, Alexandre Gret, Huub Douma, and John Scales (Ctr. for Wave Phenomena and Dept. of Geophys., Colorado School of Mines, Golden, CO 80401-1887)

Multiply scattered waves are extremely sensitive to small changes of the medium through which these waves have propagated. Coda Wave Interferometry [Science 295, 2253-2255 (2002)] is a new technique that utilizes multiply scattered waves in the time domain to monitor small changes in media. This is applied to ultrasonic waves that were recorded in a granite sample that was subjected to a change in temperature. Velocity perturbations of about $0.1 \%$ can be detected with this technique with an accuracy of about $0.02 \%$. A multiply scattered wave in an elastic medium has traveled part of its trajectory as a $P$-wave and part as an $S$-wave. A model for the equilibration of $P$ - and $S$-waves is presented. This model is used to extend the theory of coda wave interferometry to include elastic wave propagation. [Work was partially supported by the NSF (EAR-0106668 and EAR-0111804), by the U.S. Army Research Office (DAAG55-98-1-0070), and by the sponsors of the Consortium Project on Seismic Inverse Methods for Complex Structures at the Center for Wave Phenomena.]

\section{Contributed Paper}

\section{2:30}

3pPA4. Coda wave interferometry in finite solids, recovery of the $\boldsymbol{P}$ to $S$ conversion rate. Richard L. Weaver and Oleg I. Lobkis (Dept. of Theoret. \& Appl. Mech., 104 S. Wright St., Univ. of Illinois, Urbana, IL 61801, r-weaver@uiuc.edu)

Diffuse fields, which appear incoherent, nevertheless retain their deterministic character. Even wave fields that have scattered thousands of times are highly repeatable if temperature is held fixed. The temperature dependence of elastic wave speeds, and the great age of these signals can, however, lead to significant fluctuations. Our $1 \mathrm{MHz}$ transient point source in a $10 \mathrm{~cm}$ aluminum block gives rise to a complex waveform that was observed to undergo an almost pure dilation of $0.0262 \%$ per degree $\mathrm{C}$ over the range from room temperature to $40^{\circ}$. The dilation rate was determined from the cross-correlation function between time-windowed responses at different temperatures. Theoretical calculations of the shift of the correlation function were constructed in terms of a weighted average of the temperature dependencies of the individual $P$ and $S$ waves that compose 
the full field. That the $P$ and $S$ wave speeds have different dependence on temperature leads to a degradation of the strength of the correlation function maximum. The rate of degradation should be a measure of the mean lifetime of $P$ and $S$ rays against mode conversion; comparisons with theory corroborate that suggestion. [Work supported by the NSF, Grant No. CMS 99-88645.]

\title{
Session 3pSA
}

\section{Structural Acoustics and Vibration: Energy Flow Methods in Vibroacoustic Analysis and Control II}

\author{
José R. Arruda, Chair \\ Department of Computational Mechanics, University of Estadual de Campinas, Cidade University, Zeferino Vaz, \\ Campinas, SP-13083-970, Brazil
}

Invited Papers

1:00

\begin{abstract}
3pSA1. Turbulent boundary layer induced vibration up to high frequencies by means of local energy methods. Pierre Hardy, Louis Jezequel, Mohammed Ichchou, and Yves Jacques (Equipe Dynamique des Systemes et des Structures, LTDS, UMR CNRS 5513, Ecole Centrale de Lyon, 69130 Ecully, France)

The local energy method developed in the last years revealed appropriate in medium and high frequencies and supplies an accurate description of the spread of vibration and acoustic fields up to high frequencies. Our aim in the paper is to provide a complete description of the turbulent boundary layer (TBL) induced vibration by means of this method, for a simply supported thin plate. The first step in the energy method proof is the characterization of energy input from a given model of the TBL pressure interspectrum. Then, is deduced the uncoherent structural response of the panel, and the uncoherent normal mean square velocity. The latter provides, using the acoustic radiation resistance, a prediction of noise radiating by the panel up to high frequencies. Accuracy of the local energy analysis versus the usual random normal modes decomposition is demonstrated. Ultimately, a numerical parametric survey is given for various internal loss level. Precisely, the link between results provided here and SEA predictions of TBL structural induced vibration is discussed.
\end{abstract}

\section{$1: 25$}

3pSA2. Absorption coefficient and energy flow path identification by means of inverse local energy method. Pierre Hardy, Louis Jezequel, and Mohammed Ichchou (Equipe Dynamique des Systemes et des Structures, LTDS, UMR CNRS 5513, Ecole Centrale de Lyon, 69130 Ecully, France)

Estimation of the single absorption rate and of acoustical power input is currently made in reverberant rooms where a diffuse field is established. In this paper we aim at describing a method dedicated to absorption coefficients and energy flow path identification within all type of acoustic fields in medium and high frequencies, by means of an inverse local energy method. Making use of an energy integral equation with diffuse reflection, an estimator of the wall various absorption rates is built up, while the cavity is excited by a standard spherical acoustic source. A similar formulation is used to characterize a wall continuous excitation. Then it is possible to go through the measurements (pressure, intensity) and supply a detailed analysis of the wall input energy flow. This study also includes a numerical comparison between optimization methods used when trying to match the calculated field and the reference field. Among those methods, the spheric gradient proves to be efficient when compared with estimators based on other cost-functions. Sensitivity to discretization, robustness versus statistic measurement errors, and relevance of identified parameters are dealt with.

\section{Contributed Papers}

\section{$1: 50$}

3pSA3. Predicted and measured structural intensity in a plate excited by a diffuse acoustic field. Michael Daley and Stephen Hambric (Grad. Prog. in Acoust. and Appl. Res. Lab., Penn State, Appl. Sci. Bldg., State College, PA 16801)

Structural intensity (S-I) fields indicate energy source and sink regions in structures as well as energy flow paths through structures. Various S-Ibased studies have been performed for structures excited at a point or group of points. Little attention, however, has been paid to S-I fields in structures under distributed random loads, such as those due to turbulent boundary layer pressure fields and diffuse acoustic fields. Such excitations occur in common engineering problems. This study presents experimental S-I results for a thin, simply supported, rectangular plate with an attached damper excited by a diffuse acoustic field. The plates velocity response to the excitation is measured using a scanning laser Doppler vibrometer.
Finite differencing techniques are applied to the measured velocity field to yield S-I vectors. The experiments results are shown to be similar to predicted S-I fields. [Work supported by ARL Penn State.]

\section{2:05}

3pSA4. Causal energy absorption by point-attached substructures. J. Gregory McDaniel and Xianhui Li (Dept. of Aerosp. and Mech. Engr., Boston Univ., 110 Cummington St., Boston, MA 02215)

One means of attempting global vibration control when a structure is excited by an unknown transient force is to attach a substructure that optimally absorbs energy by matching the structural dynamics. Frequencydomain analysis allows one to determine the substructural admittance for optimal absorption, however the resulting admittance is often noncausal. A classic noncausal example is when the substructural admittance is chosen 
as the complex conjugate of the structural admittance. This presentation will present a methodology for solving this problem by employing an implicitly causal Fourier series in frequency for the substructural imped- ance. The Fourier coefficients of this series are chosen to maximize the energy absorption of the attachment. This methodology also allows one to seamlessly incorporate a passivity requirement for the substructure.

WEDNESDAY AFTERNOON, 4 DECEMBER 2002

GRAND CORAL 3, 1:00 TO 3:00 P.M.

\title{
Session 3pSC
}

\section{Speech Communication and Signal Processing in Acoustics: Speech and Signal Processing (Poster Session)}

\author{
Maria Garcia, Cochair \\ VAN-A, Dept. Electron., Av. San Pablo \#180, Col. Reynosa Tamaulipas, Azc. CP 02200, D.F. Mexico \\ John G. Harris, Cochair \\ Center QEFP, Northwestern University, 2137 North Sheridan Road, Evanston, Illinois 60208-3020
}

Contributed Papers

\begin{abstract}
All posters will be on display from 1:00 p.m. to 3:00 p.m. To allow contributors an opportunity to see other posters, contributors of odd-numbered papers will be at their posters from 1:00 p.m. to 2:00 p.m. and contributors of even-numbered papers will be at their posters from 2:00 p.m. to 3:00 p.m.
\end{abstract}

3pSC1. Automatic phone segment alignment using statistical deviations from manual transcriptions. Toru Hayakawa, Katsuhiko Shirai (School of Sci. and Eng., Waseda Univ., 3-4-1 Okubo, Shinjuku, Tokyo 169-8555, Japan, toru@shirai.info.waseda.ac.jp), Hiroaki Kato (ATR Human Information Sci. Labs., Kyoto 619-0288, Japan), and Yoshinori Sagisaka (GITS/GITI, Waseda Univ., Tokyo 189-0051, Japan)

For precise temporal characteristic description, disagreements between manual labeling and automatic labeling were quantitatively analyzed with respect to the spectral feature extraction, adoption of acoustic matchers (HMM models), and acoustic matcher by itself. Error analysis shows that boundaries are shifted at phone boundaries where the speech spectrum changes quite rapidly. This disagreement results from the spectral feature extraction averaged over a given window. For the adoption of model, big errors are found at phone boundaries where the spectrum changes slowly. The third model-dependent errors are seen at phones whose duration cannot be shorter than the frame increment period times the HMM state number. To take into account these error factors individually to reduce the amount of alignment errors, we modified the automatic alignment results context-dependently using statistical characteristics of phone boundary displacement. This post-processing of boundary modification reduces boundary errors from $14.79 \mathrm{~ms}$ to $11.07 \mathrm{~ms}$. Supplementary experiment shows that this improvement of about $4 \mathrm{~ms}$ corresponds to eight times of error reduction obtained by speaker adaptation of acoustic matchers. [Work supported by TAO, Japan.]

3pSC2. Duration normalization for improved automatic speech recognition. Jon P. Nedel and Richard M. Stern (Dept. of Elec. and Computer Eng. and School of Computer Sci., Carnegie Mellon Univ., Pittsburgh, PA 15213, jnedel@cs.cmu.edu)

While hidden Markov models (HMMs) serve as the basic acoustic modeling framework for many automatic speech recognition systems, they are known to model the duration of sound units poorly. Phone duration normalization can be accomplished by adding and reconstructing missing frames when a phone is shorter than the desired duration, and by deleting frames when a phone is longer than the desired duration. If phone segmentations are known a priori, this technique achieves relative reductions in word error rate (WER) of up to $35 \%$, confirming the conjecture that speech with normalized phone durations may be modeled better and dis- criminated more accurately using standard HMM acoustic models. Unfortunately, duration normalization using imperfect automatically generated phone segmentations has not yielded significant recognition improvements. A modification of the duration normalization approach has been developed. Three different feature streams are generated for each utterance using various combinations of expansion and contraction of hypothesized phone segments. Each stream is recognized using an acoustic model trained for that stream. While the resulting recognition hypotheses themselves are not significantly better than baseline, these hypotheses can be automatically combined to produce relative improvements in WER of up to $7.7 \%$ over several speech databases. [Work supported by DARPA and Telefónica.]

3pSC3. Fast on-line speaker/environment adaptation using modified maximum likelihood stochastic matching. Shubha L. Kadambe (HRL Labs., LLC, 3011 Malibu Canyon Rd., Malibu, CA 90265) and Marcus Iseli (UCLA, Westwood, CA)

The problem of speaker/environment adaptation to improve the recognition accuracy and thus making recognizers robust is addressed here. For this, a fast on-line adaptation algorithm that does not need a separate adaptation training data and that adapts acoustic models fast enough to achieve near real-time recognition is developed. This technique is based on stochastic matching in the model space similar to [A. Shankar and C.-H. Lee, IEEE Trans. Signal Process. 4, 190-202 (1996)]. For fast adaptation only the models and the mixture components that need to be adapted are selected based on the cluster formation and Euclidean distance. This adaptation algorithm is implemented as part of a GMM based continuous speech recognizer. It is tested using a non-native speakers dataset. For example, the five best hypotheses output of the speech recognizer before and after applying the adaptation technique indicated that the right answer corresponding to an utterance "none of the earth" before adaptation did not correspond to the best hypothesis; however, it corresponded to the third best. After adaptation all of the five-best hypotheses converged to the right answer. The test results of this technique on a larger non-native speakers' dataset shows $70 \%$ to $75 \%$ relative WER improvement. 
3pSC4. Cues for question intonation in Arabic: Disambiguation techniques for use in automatic speech recognizer systems. Leslie Barrett (Transclick, Inc., New York, NY 10021) and Kazue Hata (Univ. of California, Santa Barbara, CA 93106)

The focus of this study is to determine the extent to which prosodic characteristics can contribute to the improvement of speech recognition in Arabic. $F 0$ rising rate was chosen to disambiguate yes-no question from declarative sentences. In Arabic, as in English, a rising intonation indicates a yes-no question whether the question takes lexical question markers or uses an inverted word order or whether the sentence takes just a declarative form. We conducted a production study with 55 yes-no question sentences uttered by a female native speaker of Arabic. Two types of measurements were taken for $F 0$ rise rate. First, we visually obtained the best-fit rise in sentence-final position. Second, we computed the rate based on minimum and maximum $F 0$ values within the sentence-final $500 \mathrm{~ms}$. The results show that although the rise obtained from the final $500 \mathrm{~ms}$ $(0.41 \mathrm{~Hz} / \mathrm{ms})$ is different from the best-fit rise rate $(0.49 \mathrm{~Hz} / \mathrm{ms})$ ( $\mathrm{p}$ $<0.05$ ), when examining two different $F 0$ rising shapes and considering JND for the rising rate (Nabelek and Hirsh, 1962), a threshold of 0.4 $\mathrm{Hz} / \mathrm{ms}$ can be considered a threshold indicator of a yes-no question intonation for this speaker. Thus, a sufficient rise rate was extracted automatically from a fixed sentence-final duration.

3pSC5. A descriptive analysis between syllables in English and Spanish. Fabiola M. Martinez Licona, John Ch. Goddard Close, and Alma E. Martinez Licona (Dept. of Elect. Eng., Universidad Autónoma Metropolitana Iztapalapa, Mexico City, Mexico 09340, fmml@xanum.uam.mx)

In recent years different attempts have been made to incorporate temporal information longer than the phoneme into automatic speech recognizers (ASR) for English. The reason for these approaches is related to limitations which arise with existing systems based on phonemes, such as the degradation in performance of ASRs under noisy conditions and variations in pronunciation due to phoneme omission. It is conjectured that humans naturally use longer time periods, corresponding, for example, to syllables, to perceptually integerate information. In the case of Spanish, little seems to have been done in this direction for ASRs. In the present paper, a preliminary comparison is made between syllables in Spanish and English with a view to their factibility in an ASR for Spanish. In particular, a descriptive statistical analysis is conducted with a Spanish speech database to derive the most common structures of the syllables and the most common monosyllables. This is contrasted with previously found results in English. Spectrograms are also used to illustrate pertinent characteristics of Spanish. These results suggest that syllables may indeed provide useful information for an ASR in Spanish and could provide greater success than their counterparts in English. [Work supported by CONACYT under Project 31929-A.] (To be presented in Spanish.)

3pSC6. Low bit rate speech coding using spectral trajectory modeling. Sorin Dusan and James Flanagan (Ctr. for Adv. Information Processing, Rutgers Univ., 96 Frelinghuysen Rd., Piscataway, NJ 08854, sdusan@ caip.rutgers.edu)

In the U.S. Federal Standard coder for 2400 bps, a data frame containing 54 bits of encoded signal is transmitted every $22.5 \mathrm{~ms}$. In each frame, 25 bits encode the spectral features (10 Line Spectrum FrequenciesLSF). In this paper we describe a method for reducing the transmission rate while preserving most of the quality and intelligibility. This method is based on modeling the spectral trajectories with polynomial functions and on encoding these functions for segments of speech extending over multiple frames. Here 10 polynomials are computed by fitting them to the 10 LSF trajectories in the least-squares sense. Then the polynomial coefficients are encoded for the whole segment instead of directly encoding the LSF vectors. The spectral parameters are thus reduced (compressed) to $[(P+1) / N] \times 100 \%$, where $P$ represents the order of the polynomials and $N$ the number of frames for each segment. Different compression rates can be achieved. For example, for $P=5$ and $N=10$ the spectral features are encoded using $40 \%$ less bits than those required to encode $N=10 \mathrm{LSF}$ vectors. For this example, the overall transmission rate is reduced to 1956 bps, yielding an average Itakura-Saito spectral distance of 0.14 between the original and reconstructed LPC parameters computed for a typical utterance.

3pSC7. Conveying discourse structure in synthetic speech. Peter C. Gordon and Harry Halpin (Univ. of North Carolina at Chapel Hill, Chapel Hill, NC 27599-3270, pcg@email.unc.edu)

A pre-processing system for assigning prosodic characteristics of speech was created in order to investigate how synthesized speech can capture the global characteristics of discourse structure. The system allows manipulation of a variety of prosodic characteristics that have been tied to the structure of discourse above the level of the sentence, in particular: pitch fluctuation, pitch range, speaking rate, and pauses. These characteristics can be used to highlight the onset of a discourse segment, defined as a group of utterances that contribute to a single discourse purpose. After highlighting the onset of the discourse segments, these speech characteristics are progressively modified to indicate the continuity of the discourse segment. The system was evaluated by comparing it to a corpus of natural speech, the Boston Directions Corpus. That corpus has been analyzed both in terms of the informational content of the discourse and the acoustic manifestations that appear in natural speech for conveying that content. Ways in which those acoustic manifestations can be realized in natural speech are discussed.

3pSC8. Intelligibility tests for synthetic speech subjective evaluation: The semantically unpredictable sentences approach for European Portuguese. Daniela Braga, Luís Coelho, António Moura, and Diamantino Freitas (Faculty of Eng. of Univ. of OPorto, R. Dr. Roberto Frias, OPorto, Portugal)

In this paper a test proposal for European Portuguese (EP) synthetic speech quality evaluation as well as some of its application are presented. For this purpose we have built a semantically unpredictable sentences (SUS) corpus of 25 sentences with different lengths that were chosen according to the standard EP phonological, syllabic and prosodic specific features. The goal is to create a standard test linguistically focused that constitutes a base for a more accurate comparison and ranking of different synthesis techniques. The tests were conducted in two distinct conditions: one performed in a controlled environment with listeners ranging from 16 to 60 years old; the other performed through the web by a large group of listeners of different ages, each one in their own environment. The listeners had only one chance of listening to each sentence. Of course in the second case this could not be controlled. The evaluation parameters considered were the number of (1) totally well identified sentences; (2) nonidentified sentences; (3) misunderstood words in each sentence; (4) misunderstood words in the whole corpus; (5) misunderstood words according to the length of the sentence; (6) errors in the perception of a word according to its position in the sentence.

3pSC9. An estimation method for fundamental frequency and voiced segment in infant utterance. Tomohiro Nakatani, Shigeaki Amano, and Toshio Irino (NTT Commun. Sci. Labs., NTT Corp., 2-4, Hikaridai, Seika-cho, Soraku-gun, Kyoto 619-0237, Japan)

Fundamental frequency $(F 0)$ and unvoiced/voiced segment (U/V) estimation of infant utterances are important for investigating humans perception of prosodic information in an early stage of speech communication. However, this estimation process is difficult as infant utterances have several features that differ to those of adults: (1) $F 0$ has a wide range in value (200 to $2000 \mathrm{~Hz}$ ); (2) $F 0$ is unstable, for example, it discontinuously changes to its double or half value; and (3) voiced segments may have high energy in the higher frequency regions degrading U/V decisions of 
existing methods. Additionally, infant utterance data is often collected in daily child care settings which lowers the signal-to-noise ratio (SNR). To cope with these problems, a robust $F 0$ estimation method based on instantaneous frequency [Nakatani and Irino, ICSLP2002] is introduced, and a new U/V detection method is proposed. The former has a mechanism to extract accurate $F 0$ avoiding double and half pitch errors in low SNR environments. Once accurate $F 0$ is obtained, the latter method can reliably detect U/V just by examining the harmonic structure corresponding to the $F 0$. The effectiveness of this method is examined using a database devised from infant utterances in daycare settings [Amano, Kato, and Kondo, ICSLP2002].

3pSC10. Dynamic constraints on the inverse problem in speech. Khalil Iskarous (Haskins Labs., 270 Crown St., New Haven, CT 06511)

The problem of determining the area function from the speech signal has long been known to be an ill-posed problem-there are many area functions that correspond to the same speech spectrum. To make the problem well-posed, the inversion process must be constrained. In this work, inversion is performed for a dynamic formant pattern of a $\mathrm{CV}$ or a $\mathrm{VV}$ transition as a whole, rather than for a single static pattern. The input to the process are formant values along with the first and second time derivatives of each formant for each frame of the formant pattern. This provides more information about the possible area function solutions, and thereby acts as a constraint. Also the area function change during the transition is constrained to be stationary at one location in the vocal tract, while maximal change occurs at only two other locations, with the area increasing at one of the locations and decreasing at the other. This constraint is derived from an empirical study of area function change. These constraints are implemented as constraints on a Riccati recursion for the reflection coeficients. Comparison with other work on dynamic constraints on speech inversion will be provided. [Work supported by NIH.]

3pSC11. Auditorily motivated elastic spectral distance and its application to emotional morphing of portrayal speech. Hisami Matsui and Hideki Kawahara (Faculty of Systems Eng., Wakayama Univ., 930 Sakaedani, Wakayama 640-8510, Japan)

An elastic spectral distance measure based on a $F 0$ adaptive pitch synchronous spectral estimation and smoothing, that is developed for a high-quality speech modification procedure STRAIGHT [Kawahara et al., Speech Commun. 27 (1999)], is introduced to provide a basis for emotional morphing. The proposed measure is based on a smoothed nonlinear frequency mapping between the target and the original speech spectra on the ERB axis. A portrayal emotional speech database, which was recorded using professional actors and a recording studio for professional use, was designed for developing and evaluating morphing functions. Target words were recorded under four different contextual conditions (preceding, following and franking carrier sentences and isolated pronunciation), two sentence types (declarative and interrogative). Perceptual effects of constituent parameters of the proposed distance measure will be discussed based on morphing experiments using STRAIGHT procedure. [Work supported by JSPS and NIME, Japan.]

3pSC12. Evaluation of a strategy for automatic formant tracking. Terrance M. Nearey (Dept. of Linguist., Univ. of Alberta, Edmonton, AB T6G 0A2, Canada), Peter F. Assmann (Univ. of Texas, Dallas, Richardson, TX 75083), and James M. Hillenbrand (Western Michigan Univ., Kalamazoo, MI 49008)

Variations on an automatic formant tracking strategy developed at Alberta will be compared to manual formant measurements from two databases of vowels spoken by men, women, and children (in Texas or Michi- gan). "Correct" vowel formant candidates for $F 1, F 2$, and $F 3$ may be found roughly 85-90 percent of the time for adult male speakers using autocorrelation LPC with the following settings: $F 3$ maximum at 3000 $\mathrm{Hz}$, LPC order of 14, sampling rate of $10 \mathrm{kHz}$ [J. Markel and A. Gray, Linear Prediction of Speech (Springer, New York, 1975)]. Experience shows good results are also often found with females' and children's speech, provided the sampling rate and $F 3$ maximum are scaled appropriately for each speaker. Our new basic strategy involves analyzing each utterance at several distinct sampling rates and coordinated $F 3$ cutoff frequencies with a fixed LPC order. Each scaling choice provides an independent set of candidates that is post-processed by a simple tracking algorithm. A correlation measure between a spectrogram of the original signal and one resynthesized from each estimated track set is defined. This measure is combined with other heuristic figures of merit (based on, e.g., continuity, formant ranges, and bandwidths) to choose the "best" analysis.

3pSC13. Speech enhancement with beamforming enhanced by parametric spectral subtraction. Jaeyoun Cho and Ashok Krishnamurthy (Dept. of Elec. Eng., The Ohio State Univ., 2015 Neil Ave., Columbus, OH 43210, krishnamurthy.1@ osu.edu)

This paper describes a novel method of speech enhancement that combines multichannel beamforming techniques with single channel spectral subtraction. Spectral subtraction methods have been widely used for speech enhancement because they require only a single microphone and provide moderate improvements in the signal-to-noise ratio (SNR). But a major drawback of spectral subtraction is that it inevitably introduces $m u$ sical noise, which is nonstationary and very annoying. Generally, the musical noise is reduced by smoothing its spectral variance in the frequency domain. Multimicrophone beamforming techniques, on the other hand, enhance the SNR by using spatial and temporal filters to introduce spatial selectivity towards the desired signal. It is shown that these two techniques are complementary, and can be combined to provide better performance than either method alone. One approach, for example, is to use spectral subtraction as a preprocessor in each channel before beamforming. In this case, the summing process of beamforming reduces the musical noise generated by spectral subtraction. We provide examples of using this approach in an automotive application.

3pSC14. Adaptive beamformer based on average vowels/consonant spectrum weights for noisy speech recognition. Masato Nakayama, Takanobu Nishiura, and Hideki Kawahara (Grad. School of Systems Eng., Wakayama Univ., 930 Sakaedani, Wakayama 640-8510, Japan)

Background noise and reverberations seriously degrades the sound capture quality. A microphone-array is an ideal candidate for capturing distant-talking speech. With a microphone array, a desired speech signal can be acquired selectively by steering the directivity. The AMNOR (Adaptive Microphone-Array for Noise Reduction) is an adaptive beamformer proposed by Kaneda et al. In addition, as the beamformer for speech capture, S-AMNOR, the AMNOR with a long time speech spectrum was also proposed by Okada et al. However, the performance of the S-AMNOR may be further improved, if each adaptive filter for vowel and consonants could be designed with average vowels/consonants spectrum. Therefore, we propose the new AMNOR with adaptive filters for vowels/ consonants, in order to improve the signal capturing performance. We evaluated the ASR (Automatic Speech Recognition) performance with the enhanced desired signal using the adaptive filters for vowels/consonants after detecting vowels and consonants on each phoneme. As a result of evaluation experiments, by comparing the results from the proposed AMNOR and the conventional AMNOR/S-AMNOR, we could confirm that the ASR performance was improved with proposed AMNOR. [Work supported by JSPS.] 
3pSC15. Cross spectral measurement of head related speech transfer functions using speaker's own voice. Masumi Nukina and Hideki Kawahara (Grad. School of Systems Eng., Wakayama Univ., 930 Sakaedani, Wakayama 640-8510, Japan)

A cross spectrum method is applied to measure sound pressure variations around the head using the speaker's own speech sounds. The variations are represented as transfer functions from the mouth reference point to a set of measuring points. Preliminary tests indicated that there are systematic frequency response variations depending on vowel colors. This vowel color dependency was not replicated in the classical measurement of speech radiation characteristics by J. L. Flanagan. However, taking into account the large (sometimes exceeding $20 \mathrm{~dB}$ ) amount of variations, it is not likely to be negligible. A set of calibration and normalization procedures were introduced to reduce artifacts due to background noise, room acoustics, zeros in the speech spectra. A series of $M$-sequence based transfer function measurements were also conducted using a head and torso simulator to evaluate intrinsic errors in the cross spectral measurements. It was found that the standard errors in the cross spectral measurements using recorded speech sounds are around $1 \mathrm{~dB}$. Based on these reference data and confidence interval calculations based on coherence, it is safe to conclude that the vowel color dependency is significantly modifying the transfer functions. [Work supported by JSPS.]

3pSC16. Real-time magnetic resonance imaging for the study of speech production. Alain Soquet, Didier Demolin (Laboratoire de Phonologie, Universit Libre de Bruxelles, 50 av. F. D. Roosevelt, 1050 Brussels, Belgium), Peter Branderud (Stockholm Universitet, Stockholm, Sweden), Bjorn Linblom (Univ. of Texas, Austin, TX 78712), and Thierry Metens (Hopital Erasme, Universit Libre de Bruxelles, Bruxelles, Belgium)

We have shown [D. Demolin et al., C. R. Biol. 325, 111 (2002)] that it is possible to record in real-time the MR images and the speech signal. This technique opens new perspectives for the study of speech production. For acquisition of the speech signal, we have used an optical microphone. The principle of the optical microphone is as follows. A first optical fibre carries an incident light produced by a light source. The light is then reflected by a diaphragm and transmitted through a second optical fibre to a photodetector. When the diaphragm moves according to the ambient sound, it modulates the amount of light reaching the photodetector. This kind of microphone has two major advantages. First, it does not contain any metallic part; it can therefore be placed close to the lips without any danger or artefact. Second, the signal is carried with optical fibres; this allows the electronic and the recording device to be placed outside the acquisition room. Real-time MRI technique allows to explore movement of articulators involved during normal speech, while dynamic MRI relies on numerous repetition of the same sequence to reconstruct the impression of movements in time.

3pSC17. An evaluation of talker localization based on direction of arrival estimation and statistical sound source identification. Takanobu Nishiura (Faculty of Systems Eng., Wakayama Univ., 930 Sakaedani, Wakayama 640-8510, Japan) and Satoshi Nakamura (ATR Spoken Lang. Translation Res. Labs., Seika-cho, Soraku-gun Kyoto 619-0288, Japan)

It is very important to capture distant-talking speech for a hands-free speech interface with high quality. A microphone array is an ideal candidate for this purpose. However, this approach requires localizing the target talker. Conventional talker localization algorithms in multiple sound source environments not only have difficulty localizing the multiple sound sources accurately, but also have difficulty localizing the target talker among known multiple sound source positions. To cope with these problems, we propose a new talker localization algorithm consisting of two algorithms. One is DOA (direction of arrival) estimation algorithm for multiple sound source localization based on CSP (cross-power spectrum phase) coefficient addition method. The other is statistical sound source identification algorithm based on GMM (Gaussian mixture model) for localizing the target talker position among localized multiple sound sources. In this paper, we particularly focus on the talker localization performance based on the combination of these two algorithms with a microphone array. We conducted evaluation experiments in real noisy reverberant environments. As a result, we confirmed that multiple sound signals can be identified accurately between "speech" or "non-speech" by the proposed algorithm. [Work supported by ATR, and MEXT of Japan.]

\title{
Session 3pSP
}

\section{Signal Processing in Acoustics and Underwater Acoustics: Acoustic Automatic Target Recognition II}

\author{
Ning Xiang, Cochair \\ National Center for Physical Acoustics, University of Mississippi, Coliseum Drive, University, Mississippi 38677 \\ Armando Andrade, Cochair \\ Calle H, No. 11, Col. Jardines de Santa Clara, Ecatepec Edo. De. CP 55450, Mexico
}

Chair's Introduction-1:00

Invited Papers

1:05

3pSP1. Outdoor experiment results for tracking airborne high-speed broadband acoustic sources. William G. Frazier, Chad Williams, Jay E. Williams (Miltec Res. and Technol., Inc., NCPA, Coliseum Dr., University, MS 38677), and Kenneth E. Gilbert (The Univ. of Mississippi, University, MS 38677)

Recent results of tracking airborne high-speed (subsonic) broadband acoustic sources with an experimental system are presented. The system consists of three acoustic arrays, wireless telemetry systems, real-time signal processing and tracking processors, and a tactical operation center-style display unit. The acoustic signal processor consists of a coherence-based detector and generalized cross 
correlator. The real-time tracking processor uses a modified extended Kalman filter algorithm. In this particular work, the calculation of acoustic sensor-to-source-bearing information will be briefly discussed along with the operation of the source detector, which is adaptive and dependent upon both amplitude and signal coherence. Special emphasis will be placed on how the tracking algorithm is compensated to accommodate unknown and variable data latencies produced by acoustic signals propagating from distant sources that are moving at a significant fraction of the speed of sound. Experimental results include estimates of source position, speed, and heading versus time. Estimates of source state uncertainty and comparison to truth data are also provided. [Work supported by the U.S. Army Space and Missile Defense Command.]

\section{$1: 35$}

3pSP2. Audible and visual representations of the signals from a seismic landmine detection system. Waymond R. Scott, Jr. (School of Elec. and Computer Eng., Georgia Inst. of Technol., Atlanta, GA 30332, waymond.scott@ece.gatech.edu), Gregg D. Larson, and James S. Martin (Georgia Inst. of Technol., Atlanta, GA 30332)

A system using high frequency seismic waves to detect buried landmines is being investigated using both experimental and numerical models. The simplest detection cue for the localization of buried landmines has been found to be resonances of the trigger mechanism and overlying soil as these resonances create substantial localized surface displacements over the buried landmine in comparison to other buried objects such as rocks, sticks, and manmade clutter. The prototype system utilizes a noncontact radar sensor to detect surface-normal displacements and a remotely-located surface-contacting source to generate seismic waves in the ground. Processing of the measured data shows the location of mines both graphically and audibly, either of which could be used as inputs for automatic target recognition algorithms. Visual images created by a wavenumber-domain signal processing algorithm and audible representations will be compared for landmines and typical clutter objects. [Work supported by ONR and ARO.]

3pSP3. Automatic acoustic mine detection using morphological perceptions. Gerhard X. Ritter, Paul D. Gader, A. Koksal Hocaoglu, and Laurentiu Iancu (Dept. of CISE, Univ. of Florida, Gainesville, FL 32611)

Recent developments in landmine detection based on the acoustic-to-seismic coupling phenomenon have demonstrated the feasibility to detect both metallic and nonmetallic mines. In this method, a loudspeaker above the ground surface insonifies the target region of the surface. Acoustic energy is coupled into the ground producing ground vibrations. The ground vibration velocity at the ground surface is measured with a laser Doppler vibrometer producing a ground surface image whose pixel values are the measured velocity amplitudes. Vertical particle velocity amplitudes directly above a mine contrast with those of the background ("away from the mine"). Image processing techniques are used in order to automatically detect regions of interest ("possible mines"). Further image analysis methods extract geometric as well as amplitude features in order to produce four-dimensional feature vectors. These vectors are input to a novel neural network based on mathematical morphology which classifies the regions of interest into mines and false alarms. After training the network, the network correctly identified all the mines on a given test set with an extremely low alarm rate.

3pSP4. (Spectral) pattern recognition as a versatile tool towards automatic landmine detection: A new European approach. Volker Klein (Kayser-Threde GmbH, Munich, Germany, kv@Kayser-Threde.de), Peter Lutzmann (FOM Ettlingen, D-76275, Ettlingen, Germany), and Thomas Mechnig (Polytech GmbH, D-76337 Waldbronn, Germany)

A mobile acousto-optical sensor (Laser Vibrometer) is being used for the detection and discrimination of buried landmines. Analysis of measurement data, obtained in a number of field tests, reveals that buried mines (anti-tank mines as well as anti personnel mines) can reliably be discriminated from nonlethal clutter objects, such as stones, wood, cans, etc. due to their individual shapes and spectral properties. Increasing operational demands on these acoustic soundings (speed, reliability, and spatial/spectral resolution) implies the introduction of new procedures and strategies to identify and suppress misleading features as well as improved correlation of useful data. These procedures will include improved techniques for real time: (a) processing of the spectrally resolved image of the 
soil surface as well as (b) spectral analysis of the on-target soil vibrations. Image processing is comprising background linearization and suppression, shape recognition and gradient detection. Spectral analysis is including the use of look-up tables for cross-correlation and identification of characteristic features. This paper is presenting an introduction to the detection technique, sounding strategies as well as subsequent spectral data evaluation toward automated target recognition.

\title{
Session 3pUWa
}

\section{Underwater Acoustics and Engineering Acoustics: Underwater Acoustic Measurement Laboratories: New Global Perspectives of Automatic Control and Management}

\author{
Carlos Ranz-Guerra, Chair \\ Instituto de Acoustica, CSIC. C Serrano 144, 28006 Madrid, Spain
}

Chair's Introduction-1:00

Invited Papers

\begin{abstract}
1:05
3pUWa1. Remote full control, by an Internet link, of an underwater acoustics laboratory. Carlos Ranz-Guerra, Pedro Cobo-Parra, Manuel Siguero-Guerra, and Alejandro Fernandez-Fernandez (Instituto de Acustica, CSIC. C/ Serrano 144, 28006 Madrid, Spain)

The Underwater Tank Laboratory located at the Instituto de Acustica, CSIC, Madrid, has been fully reshaped. Now, the two bridges (emission and reception) have full automatic motion control by the operator. These capabilities were complemented by a new management of signal generation, signal acquisition, processing and storing of data. This new framework makes many of the tasks to be performed in this kind of facility easier by putting at the hands of the operator specific friendly software programs that attend to the main aspects of the ongoing experiment. In one step forward, the remote control of all the functionalities was considered feasible. The potentialities of the Internet were thought to provide a new dimension to the laboratory by lowering the difficulties of taking over the full control of the installation, by any user around the world. Here is one real example of how this achievement can be carried out. The Underwater Acoustics Laboratory at the Instituto de Acustica, CSIC, is now ready to be run by any one interested. The main lines, over which this problem has been considered, are described in this paper. [Work supported by PN on Science and Technology and CSIC, Spain.]
\end{abstract}

3pUWa2. Hydroacoustic station network for monitoring the Comprehensive Nuclear-Test-Ban Treaty (CTBT). Martin W. Lawrence, Marta Galindo Arranz, Patrick Grenard, and John Newton (CTBTO, Vienna Intl. Ctr., Vienna, Austria, martin.lawrence@ctbto.org)

The Comprehensive Nuclear-Test-Ban Treaty (CTBT) provides for monitoring of the whole globe by a network of stations, using various technologies, in order to verify the absence of nuclear explosion tests. The hydroacoustic component of this network, which monitors the major world oceans, is currently under construction. When complete it will consist of 11 stations located with an emphasis on the vast ocean areas of the Southern Hemisphere. Presently, three stations have been completed and work is underway on all of the remaining stations. The stations transmit real-time continuous data to the CTBT Organization headquarters in Vienna, Austria. The hydroacoustic network uses two different types of stations. One type is based on hydrophones floated from the sea floor to the SOFAR axis depth, arranged horizontally in a triplet configuration. The other type is based on the use of seismometers located on small islands to detect hydroacoustic signals after conversion to seismic signals at the flanks of the island. During the time since completing the first stations, many interesting acoustical phenomena have been observed in the data. 
3pUWa3. A network, via www, linking laboratories of underwater acoustics. Vicente Gallego (ETSI de Armas Navales, Arturo Soria, 287, Madrid 28033, Spain)

The High Technical School for Naval Weapons Engineers is a teaching institution of the Spanish Navy dedicated to training advanced engineers in the related technologies to the systems of naval weapons. Some scientific disciplines, like the underwater acoustics, are also included in its curriculum. The tasks of research are being developed by means of collaborations with civil organisms such as Institutes of Scientific Research and departments of some universities. In this context, a proposal has been prepared for creating a network for laboratories of underwater acoustics. It was initially formed by the interconnection via www of the Laboratory of Underwater Acoustics in the Naval Weapon School and the Laboratory of hydroacoustics in the Institute of Acoustics, a center of scientifical research pertaining to the Science and Technology Ministry. The joint exploitation of the diverse capabilities from different laboratories, the rational integration of activities and the saving of efforts and costs are the basic ideas for this project. In this paper are presented the general lines that have been considered in the development of this proposal, the future possibilities of using for a network like this as well as the practical limitations addressed by this new concept of networks of laboratories.

3pUWa4. Acoustical contamination: Effects on the population of mular dolphin and on their habitats. Ricardo Hernandez, Renau De Stephanis, Jose Luis Cueto, and Rivas Neus (Acoust. and Vib. Lab., Univ. of Cadiz, lav@uca.es)

In this conference we present the part of the research which has been carried out since July 2002, inside the European Project LIFE-Nature 2002 Ref. LIFE 02/ NAT/E/8610. The research aims at the anthropogenic activities existing in the research area that could affect the studied species in a different way. One of the threats is the acoustical contamination, very strong in the research area due to intensive maritime traffic, especially in the area of Gibraltar. It would be extremely useful to establish the common data base in order to permit the researchers to exchange the data obtained, tests details, and experience. In our case it would be very interesting to follow this type of marine species.

\section{$2: 25$}

3pUWa5. Ocean acoustic laboratory at the Pacific Missile Range Facility. Peter Stein, Jason Rudzinsky, Subramaniam Rajan (Sci. Solutions, Inc., 99 Perimeter Rd., Nashua, NH 03063, pstein@scisol.com), and James Lewis (Sci. Solutions, Inc., Kalaheo, HI 96741)

An ocean acoustic laboratory (OAL) is being implemented at the Pacific Missile Range Facility (PMRF). The range covers 1100 sq $\mathrm{nm}$ off the west coast of Kauai, HI, with water depths ranging from very shallow to abyssal plane. There are 172 bottom-mounted hydrophones and 15 bottom-mounted sources permanently cabled to shore. The range is used extensively for training and test and evaluation. The general OAL concept is to improve the range products by providing an accurate depiction of the four-dimensional ocean environment. A high-resolution ocean model has been implemented for the region with 48 -h forecasts available on the Scientific Solutions web site (www.scisol.com/hawaii). We are currently implementing a tomographic imaging capability using the bottommounted sources and receivers. These sound-speed images will be assimilated into the ocean model to improve accuracy. The long-term goals include providing a real-time picture of this well-described ocean environment over the global network. Applications of the ocean acoustic laboratory include oceanographic research, ocean acoustic research, system evaluation, training, and virtual war fighting. [Work supported by ONR and CEROS.]

\section{Contributed Paper}

\section{2:45}

3pUWa6. New underwater acoustic tank facility at Georgia Tech. Michael Gray, Ralph Herkert, George McCallII, Gary Caille (Georgia Tech Res. Inst., Atlanta, GA 30332, michael.gray@gtri.gatech.edu), Van Biesel, John Bogle, Jayme Caspall, Steven Hahn, Adam Lamb, Thomas Logan, James Martin, Peter Rogers, and David Trivett (Georgia Tech, Atlanta, GA 30332)

A large underwater acoustic tank facility located in the Woodruff School of Mechanical Engineering at Georgia Tech has recently been completed. The facility includes a rectangular concrete water tank 25 feet deep, 25 feet wide, and 34 feet long containing around 160,000 gallons of water. There are three computer-controlled positioners: an $x-y-z-\theta$ posi- tioner and a $z-\theta$ positioner mounted on carriages and a bottom mounted rotator. The facility has a large rectangular nearfield array which can be used either as a receiver or a transmitter. A single vertical nearfield line array can be translated by the $x-y$ positioner to synthesize a cylindrical nearfield receiving array. The rectangular nearfield transmitting array and the synthesized cylindrical receiving array were designed to be used with the bottom mounted rotator to measure the true farfield bistatic target strength of any target up to one meter in length as a function of the target aspect angle. Such measurements can be done from $2 \mathrm{kHz}$ to over $10 \mathrm{kHz}$. The tank is being used for transducer development, materials, and flow noise studies in addition to structural acoustics. Several available multichannel data acquisition systems will be described. [Work supported, in part, by a DURIP grant from ONR.]

3:00-3:15

Panel Discussion 


\title{
Session 3pUWb
}

\section{Underwater Acoustics: General Topics in Underwater Acoustics I}

\author{
George E. Ioup, Chair \\ Department of Physics, University of New Orleans, New Orleans, Louisiana 70148
}

\section{Contributed Papers}

\begin{abstract}
1:00
3pUWb1. Use of the internet in maintaining and operating the Intermediate Scale Measurement System (ISMS) underwater test facility. Duane Nightingale, Steve Troxel, and Bruce Libby (Naval Surface Warfare Ctr., Acoust. Res. Det., 33964 N. Main St., Bayview, ID 83803, nightingaled1@nswccd.navy.mil)
\end{abstract}

The Intermediate Scale Measurement System (ISMS) is the Navy's premier underwater measurement facility for performing target strength and structural acoustic evaluations on 1/4 scale submarine models. Located in Bayview, Idaho, ISMS reaches out to its customers through extensive use of the internet and open source technologies. Central to the operation of the system is an apache web server used to monitor and control systems within the models and the land-based facilities. Many aspects of ISMS, including data acquisition can be performed remotely over the internet. Specific topics include overcoming firewall issues, remote monitoring and control, collaborative test development and execution, and open source tools.

\section{1:15}

3pUWb2. Generic file format. Nick Felgate (Defence Sci. and Technol. Lab., Farnborough, Hampshire GU14 OLX, UK)

The Generic File Format (GFF) is a file format developed within the UK ASW community for the interchange and storage of underwater sonar data. Originally developed for the interchange of time-series data between analysis systems, it has been extended to provide for storage of processed acoustic data (e.g., power and DEMON spectrum, lofargram grey-scale), nonacoustic data (e.g., own-ship dynamics, sensor configuration) and event data (e.g., tracker output, sonar intercepts). The format employs the chunk concept, as used in the WAV and AIFF file formats, to provide extendability (including local variants) while providing a measure of backward compatability. However, the basic concept has been adapted to allow for the mixing in the one file of multiple channels of different sample-rates and data-types through the inclusion of a data frame concept and multiple data blocks. Chunk cross-referencing has been employed to ensure data consistency. A provision is made in the header of the file to store details of the sensor and processing for the data (e.g., the number of hydrophones, beam direction, FFT size) so that an analysis system does not need to know about the sensor or other system from which the data originated.

\section{$1: 30$}

3pUWb3. Wavelet denoising of underwater acoustic data. Juliette W. Ioup and George E. Ioup (Dept. of Phys., Univ. of New Orleans, New Orleans, LA 70148)

The Littoral Acoustic Demonstration Center (LADC) deployed three Environmental Acoustic Recording System (EARS) buoys in the northern Gulf of Mexico during the summer of 2001. The buoys recorded frequencies up to $5859 \mathrm{~Hz}$ continuously for 36 days. The acoustic signals measured include sperm whale vocalizations and seismic airguns. The data are analyzed using time series, Fourier transforms, and spectrograms. Noise removal or denoising of these data at selected times using wavelets is investigated. The effectiveness of the technique is influenced by the choice of wavelet, the decomposition level, and the threshold amplitude. Results will be presented of wavelet denoising of the LADC Summer 01 data and compared with Fourier noise removal methods including bandpass filtering and thresholding. [Research supported by ONR.]

\section{1:45}

3pUWb4. Organic sonobuoy ranging. Nick Felgate (Defence Sci. and Technol. Lab., Farnborough, Hampshire GU14 OLX, UK)

It is important that military vessels periodically check their passive signatures for vunerabilities. Traditionally, this is undertaken on a fixed range (e.g., AUTEC, BUTEC) with low noise conditions. However, for 
operational and cost reasons it is desirable to be able to undertake such measurements while the asset is operating in other areas using expendable buoys deployed by the vessel itself. As well as the wet-end hardware for such organic sonobuoy ranging systems (e.g., calibrated sonobuoys, calibrated data uplink channels), careful consideration is needed of the signalprocessing required in the harsher environmental conditions of the open ocean. In particular, it is noted that the open ocean is usually much noisier, and the propagation conditions more variable. To overcome signal-tonoise problems, techniques such as Doppler-correction, zero-padding/ peak-picking, and noise estimation/correction techniques have been developed to provide accurate and unbiased estimates of received levels. To estimate propagation loss for source level estimation, a model of multipath effects has been included with the ability for analysts to compare predicted and observed received levels against time/range and adjust modeling parameters (e.g., surface loss, bottom loss, source depth) to improve the fit.

\section{2:00}

3pUWb5. A resonant scattering formalism for a fluid loaded elastic spherical near a planar boundary. Garner Bishop (Naval Undersea Warfare Ctr. Div. Newport, Newport, RI 02840, bishopgc@npt.nuwc.navy.mil)

Free field scattering from stationary fluid loaded elastic targets is well known and understood in terms of free field resonant scattering theory (RST), that it is given by the superposition of resonant and background components, and that the resonant components can be isolated by subtraction of an appropriate background. Backgrounds for isolating free field resonance spectra are well known. However, RST for scattering from a fluid loaded elastic target near a planar boundary where target scattering is accompanied by target-boundary scattering is less well known. A $T$-matrix formalism for plane wave scattering from rigid and soft spheres near a planar penetrable boundary is generalized to include a fluid loaded elastic spherical shell and RST is used to replace the free field $T$-matrix for the elastic shell that occurs within the formalism. It is shown that targetboundary scattering couples resonant and background components, couples modal resonances, and produces super-resonances. A background is introduced and it is shown that while background scattering is suppressed, coupled background and resonant scattering is not. Numerical results demonstrate some of the effects of target boundary scattering on the free field resonant spectrum of the shell as well as the dependence of target-boundary scattering on target.
2:15

3pUWb6. Pressure and velocity fields produced by an underwater explosion. Kendall S. Hunter and Thomas L. Geers (Dept. of Mech. Eng., UCB427, Univ. of Colorado, Boulder, CO 80309)

A model for a moderately deep underwater explosion bubble has recently been developed that integrates the initial shock wave phase and the subsequent oscillation phase of the motion [T. L. Geers and K. S. Hunter, J. Acoust. Soc. Am. 111, 1584-1601 (2002)]. A hyperacoustic volumeacceleration model for the shock wave phase employs an empirical pressure-profile expression for the far field to determine bubble radius as a function of time, thereby providing initial conditions for the subsequent oscillation phase. The model for the oscillation phase yields bubbleresponse equations that incorporate first-order wave effects in both the external liquid and the internal gas. In this paper, a radiation model consistent with the bubble model is formulated and evaluated. The far-field pressure and velocity fields produced during the shock wave phase derive from the previously mentioned pressure-profile expression. Those produced during the oscillation phase derive from a matched-asymptoticexpansion solution for a translating acoustic source/dipole [F. G. Leppington and H. Levine, Proc. R. Soc. London, Ser. A 412, 199-221 (1987)]. Computed far-field pressure and velocity histories and snapshots are shown. Comparisons with existing data exhibit good agreement. [Research sponsored by ONR.]

\section{$2: 30$}

3pUWb7. Utilizing acoustic suspended-sediment measurement techniques in laboratory flumes. Daniel Wren, Srikanth Vadakapurapu, James Chambers (Natl. Ctr. for Physical Acoust., Univ. of Mississippi, 1 Coliseum Dr., University, MS 38677), Roger Kuhnle (USDA-ARS Natl. Sedimentation Lab., Oxford, MS 38655), and Brian Barkdoll (Univ. of Mississippi, University, MS 38677)

Automated measurement of suspended sediments is crucial to the study of sediment transport. The short duration, high-intensity flows that are responsible for a large fraction of sediment movement are best observed by continuous monitoring systems. Acoustic systems are ideal for continuous monitoring and add the advantage of nonintrusively measuring through the water column. In the present work, a single-frequency acoustic system for measuring suspended-sediment concentration in fluvial environments is described. The equipment and procedures used in developing hardware and software for the acoustic technique in two sets of laboratory flume experiments will be presented. Both implicit and explicit methods were used to convert backscatter data into sediment concentrations. The implicit method yielded $39 \%$ error and the explicit, $20 \%$. 


\section{Plenary Session and Awards Ceremony}

\section{Presentation of Certificates and Awards by Acoustical Society of America}

Richard Stern, President

\begin{tabular}{ll}
\multicolumn{2}{l}{ Presentation of Certificates to New Fellows } \\
Richard H. Campbell & William C. Moss \\
Laurel H. Carney & Philip A. Nelson \\
Bruce D. Cornuelle & Marshall H. Orr \\
Peter H. Dahl & Jack E. Randorff \\
George E. Ioup & Sean F. Wu \\
Leon M. Keer & Ning Xiang \\
Hugh J. McDermott & George Zweig \\
Colette M. McKay &
\end{tabular}

Presentation of Awards

Science Writing Award in Acoustics for Journalists to Bennett Daviss for his article Snap, Crackle and Pop published in New Scientist, 21 July 2001

Science Writing Award for Professionals in Acoustics to Sharem Vaezy for his article (coauthored with Roy W. Martin and Lawrence A. Crum) Acoustic Surgery in Physics World, August 2002

Pioneers of Underwater Acoustics Medal to Frederick D. Tappert

Silver Medal in Noise to Louis C. Sutherland

Wallace Clement Sabine Medal to Alfred C. C. Warnock

Honorary Fellowship to Michael Longuet-Higgins

\section{Presentation of Awards by Mexican Institute of Acoustics}

Sergio Beristain, President

John William Strutt, 3rd Baron of Rayleigh Medal to Leo L. Beranek

John William Strutt, 3rd Baron of Rayleigh Medal to Per V. Brüel

Herman Ludwig Ferdinand von Helmholtz Medal to José Luis Rodríguez García

\section{Presentation of Certificates by Iberoamerican Federation of Acoustics}

Samir N. Y. Gerges, President 